\title{
Self-Assembly of Fibronectin Mimetic Peptide-Amphiphile Nanofibers
}

\author{
A THESIS \\ SUBMITTED TO THE FACULTY OF THE GRADUATE SCHOOL \\ OF THE UNIVERSITY OF MINNESOTA \\ BY
}

EMILIE LYNN REXEISEN

IN PARTIAL FULFILLMENT OF THE REQUIREMENTS

FOR THE DEGREE OF DOCTOR OF PHILOSOPHY

\author{
Efrosini Kokkoli, Advisor
}

June 2010 
(c) Emilie Lynn Rexeisen 2010 


\section{Acknowledgements}

I have thoroughly enjoyed my graduate career in Minnesota. The time has been marked by many life changes. For the first time, I did not file taxes as a dependent of my parents. I got married. We bought a house. And we are expecting our first child. I am so glad to have met so many wonderful people to help me out along the way.

First and foremost, I must thank my advisor, Efrosini Kokkoli, for introducing me to such an interesting project. I have learned a great deal under her tutelage that will help me in the rest of my career. Thank you for your support and advice.

I was supported during my graduate career by several funding sources including the Edward Howard and Mary Cox Fellowship from the University of Minnesota, the NIH Biotechnology Training Grant, and NSF. Thank you for making my graduate career possible. Special thanks to the Biotechnology Training Grant, for allowing me to participate in a summer internship at Medtronic and introducing me to graduate students in different departments.

The members of the Kokkoli group have been a fantastic resource for learning new techniques and for troubleshooting project difficulties. Thanks to Ana Mardilovich, Jenn Craig, and Ashish Garg for getting me started in my graduate studies. And to Kamlesh Shroff for his help these last several months. And thanks to the rest of the Kokkoli group: Todd, Alison, Döne, Brett, Matt, Nicole, Maroof, and, Tim for your lab support and friendship.

I am privileged to have received much research help from the excellent graduate students and staff at the University of Minnesota. Rajiv 
Taribagil spent many an afternoon analyzing the SANS data with me. Wei Fan and Todd Pangburn took the cryo-TEM images of my samples. Many thanks to David Giles for lending his rheology expertise and teaching me how to use the rheometer. Dan Bolintineanu was gracious enough to help me make the peptide-amphiphile images.

I have met the best group of friends at Minnesota. I would never have believed that I could have enjoyed graduate level classes as much as I did, and the only reason was learning with all my classmates. I cannot thank them all enough for making these years some of the best in my life. Special thanks to Will and Alison, thank you for all the dinners and late night board games. We will miss you guys and wish you the best of luck!!!

I wouldn't be here today without the love and support of my parents: Mary and Stephen Grzywa. Thank you for always encouraging me to dream and giving me the resources to pursue my dreams. I cannot thank you enough. Thank you to my siblings, Ken, Matt, and Steph. I loved growing up with each of you, (despite the inevitable sibling squabbles). And thank you to the rest of my extended family and in-laws for listening and nodding in good humor whenever I tried to explain what my research project is all about.

And finally, to my husband, Jon. I love you more than words can say. I am so blessed to have you in my life. Thank you for all the support you have given me during graduate school, especially with your expert picture drawing! Thank you for all the goofy sing-a-longs, Trivial Pursuit questioning, horn duets, and camping trips. I look forward to spending the rest of my life with you and all the experiences to come. 
For my husband, Jonathan Rexeisen and my parents, Stephen and Mary Grzywa I am so blessed to be surrounded by your love. 


\section{Abstract}

Many therapeutic strategies incorporate peptides into their designs to mimic the natural protein ligands found in vivo. A few examples are the short peptide sequences RGD and PHSRN that mimic the primary and synergy-binding domains of the extracellular matrix protein, fibronectin, which is recognized by the cell surface receptor, $a_{5} \beta_{1}$ integrin. Even though scaffold modification with biomimetic peptides remains one of the most promising approaches for tissue engineering, the use of these peptides in therapeutic tissue-engineered products and drug delivery systems available on the commercial market is limited because the peptides are not easily able to mimic the natural protein. The design of a peptide that can effectively target the $a_{5} \beta_{1}$ integrin would greatly increase biomimetic scaffold therapeutic potential. A novel peptide containing both the RGD primary binding domain and PHSRN synergy-binding domain for fibronectin joined with the appropriate linker should bind $a_{5} \beta_{1}$ integrin more efficiently and lead to greater cell adhesion over RGD alone.

Several fibronectin mimetic peptides were designed and coupled to dialkyl hydrocarbon tails to make peptide-amphiphiles. The peptides contained different linkers connecting the two binding domains and different spacers separating the hydrophobic tails from the hydrophilic headgroups. The peptide-amphiphiles were deposited on mica substrates using the Langmuir-Blodgett technique. Langmuir isotherms indicated that the peptide-amphiphiles that contained higher numbers of serine residues formed a more tightly packed monolayer, but the increased number of serines also made transferring the amphiphiles to the mica substrate more difficult. Atomic force microscopy (AFM) images 
of the bilayers showed that the headgroups might be bent, forming small divots in the surface. These divots may help expose the PHSRN synergy-binding domain. Parallel studies undertaken by fellow group members showed that human umbilical vein endothelial cells and $a_{5} \beta_{1}$ integrins immobilized on an AFM tip preferred binding to a fibronectin mimetic peptide that contained both hydrophilic and hydrophobic residues in the linker and a medium length spacer.

Most cells require a three-dimensional scaffold in order to thrive. To incorporate the fibronectin mimetic peptide into a three-dimensional structure, a single hydrocarbon tail was attached to form a peptideamphiphile. Single-tailed peptide-amphiphiles have been shown to form nanofibers in solution and gel after screening of the electrostatic charges in the headgroup. These gels show promise as scaffolds for tissue engineering. A fibronectin mimetic peptide-amphiphile containing a linker with alternating hydrophobic and hydrophilic residues was designed to form nanofibers in solution. The critical micelle concentration of the peptide-amphiphile was determined to be $38 \mu \mathrm{M}$, and all subsequent experiments were performed above this concentration. Circular dichroism (CD) spectroscopy indicated that the peptide headgroup of the peptide-amphiphile forms an $\alpha+\beta$ secondary structure; whereas, the free peptide forms a random secondary structure. Cryogenic-transmission electron microscopy (cryo-TEM) and small angle neutron scattering showed that the peptide-amphiphile self-assembled into nanofibers. The cryo-TEM images showed single nanofibers with a diameter of $10 \mathrm{~nm}$ and lengths on the order of microns. Images of higher peptideamphiphile concentrations showed evidence of bundling between individual nanofibers, which could give rise to gelation behavior at higher concentrations. The peptide-amphiphile formed a gel at concentrations 
above $6 \mathrm{mM}$. A $10 \mathrm{mM}$ sample was analyzed with oscillating plate rheometry and was found to have an elastic modulus within the range of living tissue, showing potential as a possible scaffold for tissue engineering. 


\section{Table of Contents}

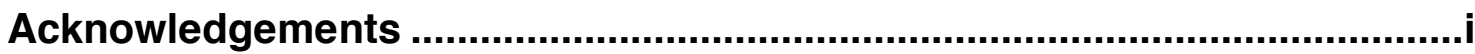

Dedication

Abstract

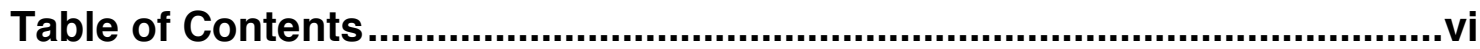

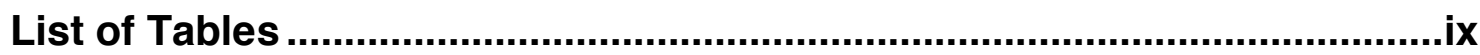

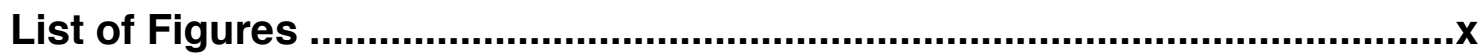

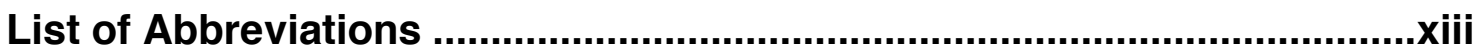

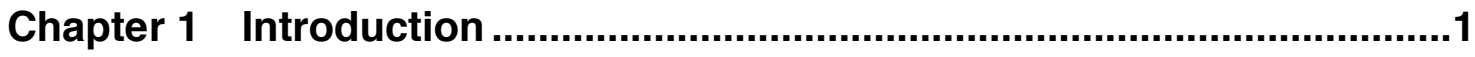

1.1 Extracellular Matrix and Cellular Adhesion .................................................2

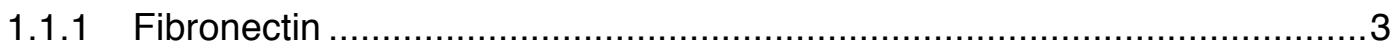

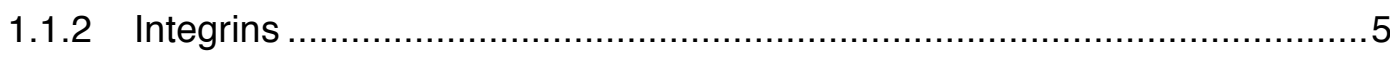

1.2 Cell Adhesion to Engineered Scaffolds .....................................................

1.3 Other Scaffold Design Considerations........................................................11

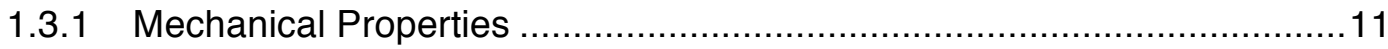

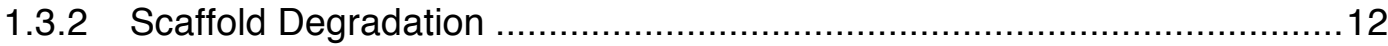

1.3.3 Encouraging Angiogenesis and Cell Migration ....................................13

1.4 Peptide-Amphiphiles ........................................................................13

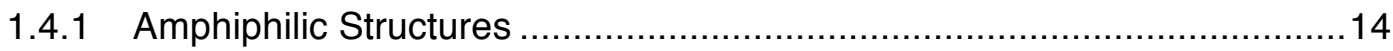

1.4.2 Applications for Peptide-Amphiphiles in the Biomedical Field ..................16

1.5 Organization of Thesis ................................................................................18

Chapter 2 Fibronectin Mimetic Peptides....................................................20

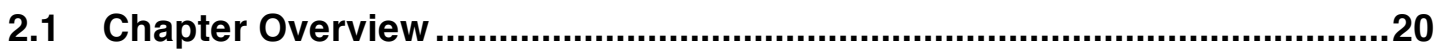

2.2 Biomimetic Peptides RGD and PHSRN ...................................................20

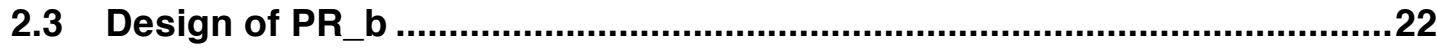

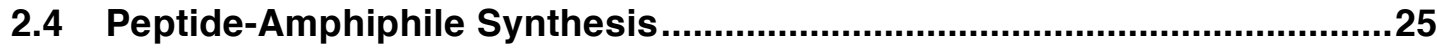

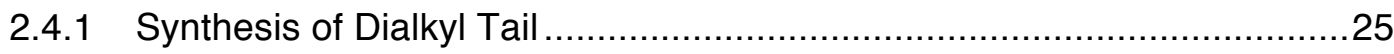


2.4.2 Solid Phase Peptide-Amphiphile Synthesis .....................................27

2.4.3 Cleaving from the Solid Support .....................................................29

2.4.4 Purification of Crude Product .............................................................. 30

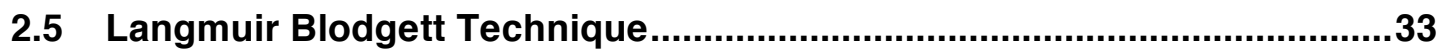

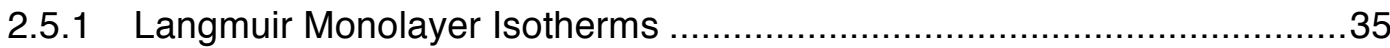

2.5.2 LB Isotherms of Fibronectin Mimetic Peptide-Amphiphiles.....................37

2.5.3 Langmuir-Blodgett Deposition............................................................

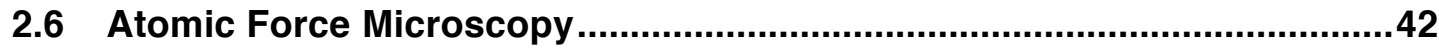

2.6.1 Atomic Force Microscopy Fundamentals ..............................................43

2.6.2 AFM Imaging of Fibronectin Mimetic Peptide-Amphiphile Langmuir Blodgett Supported Bilayers........................................................4 46

2.7 Cell and Integrin Adhesion to PR_a - PR_f Langmuir-Blodgett Supported Surfaces .........................................................................................49

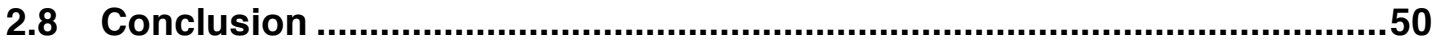

Chapter 3 Peptide-Amphiphile Nanofibers ...........................................52

3.1 Chapter Overview ....................................................................................52

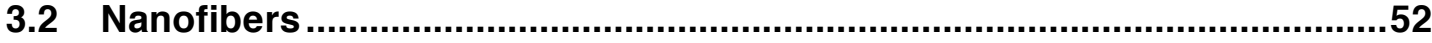

3.2.1 Mechanisms of Self-Assembly ...................................................... 53

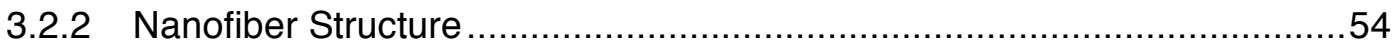

3.2.3 Peptide Headgroup Secondary Structure ..............................................57

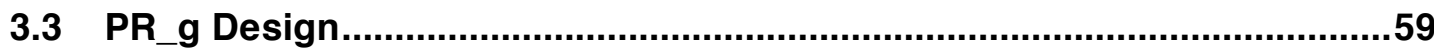

3.3.1 PR_g Binding Domains, Linker Region, and Tail..............................60

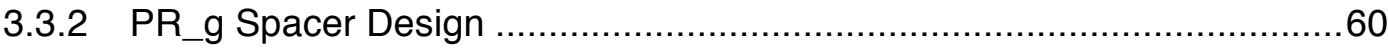

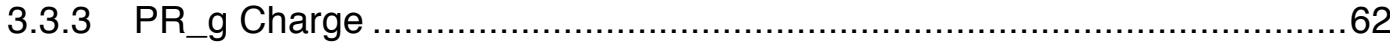

Chapter 4 Synthesis \& Characterization of PR_g.................................64

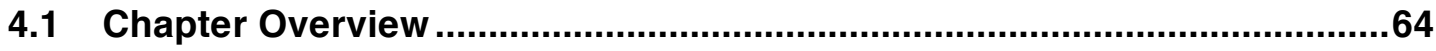

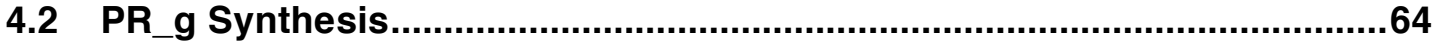

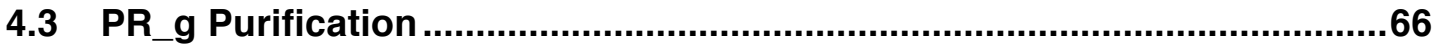

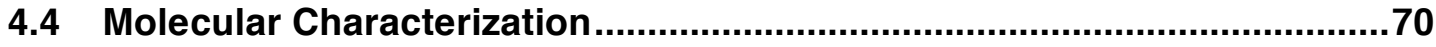

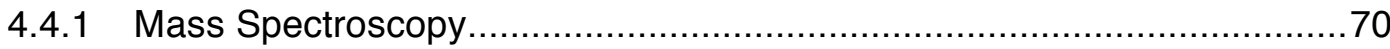




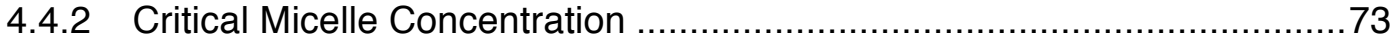

4.4.3 Zeta Potential ............................................................................... 75

4.5 Nanofiber Structural Characterization ........................................................77

4.5.1 Circular Dichroism Spectroscopy .................................................... 77

4.5.2 Cryo-Transmission Electron Microscopy ........................................ 84

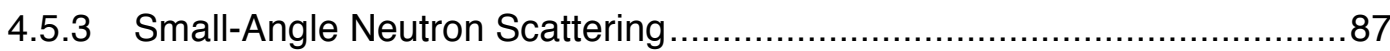

4.6 PR_g Nanofiber Gel Characterization .........................................................94

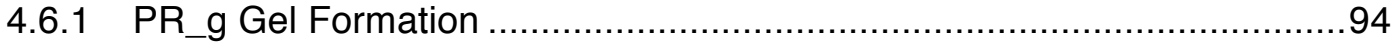

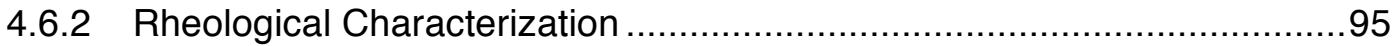

Chapter 5 Conclusion..........................................................................102

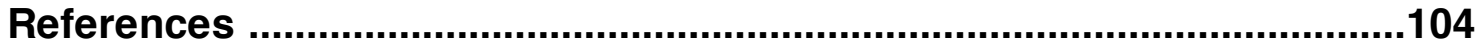




\section{List of Tables}

Table 1-1 A few biomimetic peptide sequences, the proteins from which they are derived, and cell receptors ...............................10

Table 2-1 Names and peptide sequences used in this study .........................23

Table 2-2 HPLC gradient used in purification of dialkyl peptide-amphiphiles ............................................................................31

Table 2-3 Depth of divot features in the AFM images of the fibronectin mimetic peptide-amphiphile films. 48

Table 4-1 HPLC gradient for purification of PR_g with acetonitrile 67

Table 4-2 HPLC gradient for purification of PR_g with methanol 68

Table 4-3 Coherent scattering lengths for selected atoms 89 


\section{List of Figures}

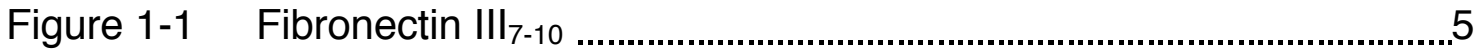

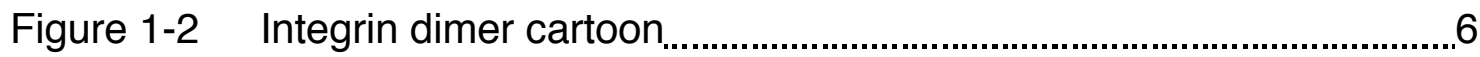

Figure 1-3 Integrin family ...............................................................................

Figure 1-4 Diagram cutaway of a micelle, vesicle, cylindrical

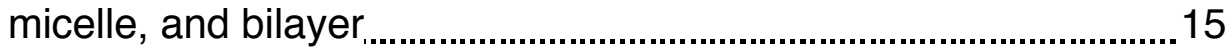

Figure 2-1 PR_b fibronectin mimetic peptide-amphiphile

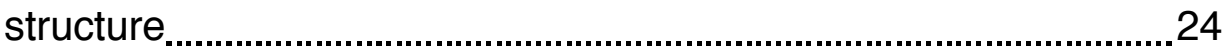

Figure 2-2 Dialkyl tail synthesis ....................................................................26

Figure 2-3 Coupling hydrocarbon tail and cleaving peptideamphiphile from resin bead...........................................................29

Figure 2-4 MALDI spectrum of purified PR_b peptide-

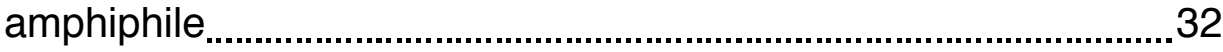

Figure 2-5 Langmuir-Blodgett trough schematic ...............................................34

Figure 2-6 Example pressure-area isotherm for a typical surfactant with labeled domains 37

Figure 2-7 LB isotherms of PR_a-PR_f ..............................................................39

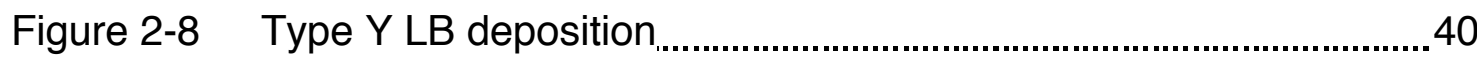

Figure 2-9 Average transfer ratios for the peptide-amphiphiles and DSPE lipid................................................................................... 42

Figure 2-10 AFM schematic ........................................................................... 44

Figure 2-11 AFM tapping mode height images of the peptideamphiphile bilayers 47

Figure 3-1 Schematic of self-assembly of peptide-amphiphile molecules into a nanofiber 56

Figure 3-2 PR_g fibronectin mimetic peptide-amphiphile structure 59

Figure 3-3 Theoretical charge of PR_g at various $\mathrm{pH}$ 
Figure 4-1 Single-tail peptide-amphiphile tail coupling and cleavage from PAL resin bead ............................................................65

Figure 4-2 Crude PR_g HPLC chromatogram ...................................................69

Figure 4-3 Pure PR_g HPLC chromatogram.................................................... 70

Figure 4-4 ESI spectrum of pure PR_g........................................................ 71

Figure 4-5 ESI spectrum of pure PR_g zoomed in on the region containing $P R \_g$ and $P R \_g$ derivatives 73

Figure 4-6 Critical micelle concentration of PR_g determined by solubilization of Nile red in PR_g micelles 75

Figure 4-7 Arrangement of ions surrounding a negative particle 76

Figure 4-8 Circularly polarized light forms a helix in space as it travels 78

Figure 4-9 CD spectra of peptides exhibiting an a-helix, $\beta$ sheet, $\beta$-turn, and P2 conformations ................................................ 79

Figure 4-10 CD spectrum of egg white lysozyme..............................................8

Figure 4-11 CD spectroscopy of PR_g peptide and peptideamphiphile (PA) 82

Figure 4-12 CD spectroscopy of a $100 \mu \mathrm{M}$ PR_g amphiphile solution diluted from a $10 \mathrm{mM}$ stock solution 83

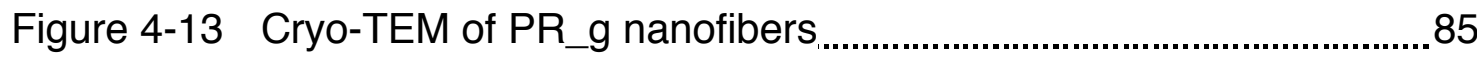

Figure 4-14 SANS sample scattering schematic ................................................8

Figure 4-15 SANS scattering intensity as a function of $q$ for a sphere, rod, and disc

Figure 4-16 SANS data for PR_g peptide-amphiphile solutions at $1 \mathrm{wt} \%$ and $0.75 \mathrm{wt} \%$ normalized with respect to concentration. 92 
Figure 4-17 SANS data of $1 \mathrm{wt} \%$ (4.12 mM) PR_g amphiphile dissolved in $100 \% \mathrm{D}_{2} \mathrm{O}$ plotted as intensity $(/(q))$

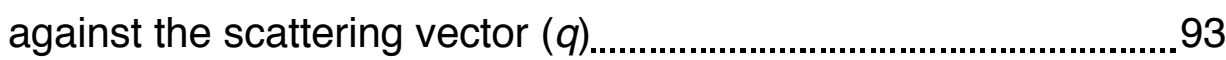

Figure 4-18 PR_g peptide-amphiphile self-supporting gel...............................94

Figure 4-19 Stress-strain curve for PR_g to determine linear viscoelastic regime ............................................................................ 97

Figure 4-20 PR_g gelation of a $12 \mathrm{mM}$ sample in Milli-Q water monitored by oscillating plate rheometry at a frequency of $1 \mathrm{rad} / \mathrm{s}$ 98

Figure 4-21 Frequency sweep showing $G^{\prime}$ and $G^{\prime \prime}$ of a PR_g gel. 


\section{List of Abbreviations}

\begin{tabular}{|c|c|}
\hline $\mathrm{ACN}$ & acetonitrile \\
\hline AFM & atomic force microscopy \\
\hline CD & circular dichroism \\
\hline CMC & critical micelle concentration \\
\hline cryo-TEM & cryogenic transmission electron microscopy \\
\hline DCM & dichloromethane \\
\hline DIPEA & diisopropylethylamine \\
\hline DMF & dimethylformamide \\
\hline DNA & deoxyribonucleic acid \\
\hline DSPE & 1,2-disteraroyl-sn-glycero-3-phosphoethanolamine \\
\hline ECM & extracellular matrix \\
\hline ESI-MS & electrospray ionization mass spectroscopy \\
\hline Fmoc & fluorenylmethoxycarbonyl \\
\hline FT-IR & Fourier transform - infrared spectroscopy \\
\hline GAG & glycosoaminoglycan \\
\hline HBTU & $\begin{array}{l}O \text {-(benzotriazole-1-yl)- } N, N, N^{\prime}, N^{\prime \prime} \text {-tetramethyluronium } \\
\text { hexofluorophosphate }\end{array}$ \\
\hline HPLC & high performance liquid chromatography \\
\hline HUVEC & human umbilical vein endothelial cell \\
\hline Ig-like CAM & immunoglobulin-like cell adhesion molecule \\
\hline IPA & isopropanol \\
\hline IR & infrared \\
\hline LB & Langmuir-Blodgett \\
\hline MALDI & matrix-assisted laser desorption/ionization \\
\hline MALDI-TOF & matrix-assisted laser desorption/ionization time-of-flight \\
\hline $\mathrm{MeOH}$ & methanol \\
\hline MMP2 & matrix metallopeptidase 2 \\
\hline
\end{tabular}




$\begin{array}{ll}\text { MRI } & \text { magnetic resonance imaging } \\ \text { NIST } & \text { National Institute of Standards and Technology } \\ \text { NMR } & \text { nuclear magnetic resonance } \\ \text { PEG } & \text { poly(ethylene glycol) } \\ \text { PGA } & \text { poly(glycolic acid) } \\ \text { PHSRN } & \text { proline-histidine-serine-arginine-asparagine } \\ \text { RGD } & \text { arginine-glycine-aspartic acid } \\ \text { SAM } & \text { self-assembling monolayer } \\ \text { SANS } & \text { small-angle neutron scattering } \\ \text { SLD } & \text { scattering length density } \\ \text { SPM } & \text { scanning probe microscopy } \\ \text { STM } & \text { scanning tunneling microscopy } \\ \text { TEM } & \text { transmission electron microscopy } \\ \text { TFA } & \text { trifluoroacetic acid } \\ \text { THF } & \text { tetrahydrofuran } \\ \text { TIS } & \text { triisopropylsilane } \\ \text { TsOH } & \text { p-toluenesulfonic acid } \\ \text { VEGF } & \text { vascular endothelial growth factor }\end{array}$




\section{Chapter 1 Introduction}

The combined impact of advances in genomics, proteomics, stem cell technology, tissue engineering, and drug delivery on our healthcare industry will radically change our world just as the recent advances in computer technology have redefined how we interact, manage data, and do business. In current medical practice, diseased or wounded tissues are replaced by organ transplantation, reconstructive surgery, and the use of prosthetics and implants. Many different organs can be transplanted to replace diseased tissue in a patient including the heart, lungs, liver, kidney, pancreas, small intestine, cornea, and skin. ${ }^{1}$ While organ transplant offers treatment for many different diseases, organ donors are in short supply. ${ }^{2}$ As of June 2010, over 107,000 people in the United States are on the organ transplant list awaiting organs, and in all of 2009, fewer than 30,000 transplants surgeries were performed. ${ }^{1}$ The lack of organ donors consequently leads to long waiting times on the organ donor list, and many patients' conditions worsen and may become fatal. Reconstructive surgery is a popular method for repairing torn ligaments and replacing damaged blood vessels using tissue from the donor's own body, but the surgery requires two separate surgeries first to harvest the donor site tissue and then to implant it in the new location. This introduces the risk of donor site morbidity. Implants are widely used in joint replacements, but due to wear of the implant and poor biocompatibility, they have a finite lifespan. One solution to the problems associated with organ transplantation, reconstructive surgery, and implants is to engineer replacement organs, blood vessels, and joints through tissue engineering.

Tissue engineering uses live cells as a replacement for diseased tissue in an external support device or as a model system to help determine the response of 
a tissue to a drug. This interdisciplinary field merges principles from both engineering and the life sciences to create a biological substitute to restore, maintain, and improve tissue function. ${ }^{2}$ The three main components of an engineered tissue mimic the three components of native tissues: the cells, the scaffold, and the soluble signals such as mitogens or growth factors. Of the three components, the scaffold requires the most engineering design. This project focuses on developing a fibronectin mimetic peptide sequence for optimal cell adhesion and designing peptide-amphiphiles that may provide a novel scaffold for tissue engineering applications.

\subsection{Extracellular Matrix and Cellular Adhesion}

Biology offers a template for developing tissue engineering scaffolds and can be used as a source of design inspiration. Tissues are primarily comprised of cells and the extracellular matrix (ECM). The ECM is a network of secreted macromolecules and serves to provide structure and support to the tissue, provide anchorage for cells, separate different tissue types, modulate cell behavior, and sequester growth factors. ${ }^{3}$ Organisms contain many different tissue types including muscle, nervous, connective, and epithelial tissues. Each tissue type has a unique organizational structure. For example, connective tissues are primarily composed of ECM proteins with very few cells; whereas, epithelial tissue is made of many cells in sheets attached to a very thin basement membrane of ECM proteins.

The ECM macromolecules fall into two basic categories: proteoglycans and proteins. Proteoglycans have a protein core with polysaccharides surrounding the protein like a brush. The polysaccharides are made of glycosaminoglycans (GAGs) such as heparin sulfate, chondroitin sulfate, and keratin sulfate. GAGs are highly hydrated due to a net negative charge and form a gel-like substance in the tissue. The GAG gel resists compressive force to give some strength to 
the tissue, yet also allows rapid diffusion of oxygen and nutrients due to the high hydration. ${ }^{3}$ Hyaluronic acid is another type polysaccharide that helps form the hydrated gel, but it is not found as a proteoglycan.

Fibrous proteins are found embedded in the GAG gel and provide further support to the tissue. The most abundant protein in vertebrates is collagen. ${ }^{3}$ It is the major stress-bearing component of connective tissue and is found extensively in bone, teeth, cartilage, tendons, ligaments, skin, and blood vessels. Elastin is another fibrous protein that gives resilience to tissues allowing them to retain their shape after deformation. ${ }^{3}$ Other common proteins in the ECM contain many adhesion domains for cells including fibronectin, laminin, vitronectin, and fibrinogen.

Many cell processes are regulated by signals that cells receive from contacts they form with nearby cells and the ECM. Cell surface receptors called cadherins, immunoglobulin-like cell adhesion molecules (Ig-like CAMs), claudins, selectins, and connexins are involved in cell-cell adhesion. ${ }^{3}$ Cellmatrix adhesion is regulated by a family of cell surface receptors called integrins. When cells bind to a substrate, they form focal adhesions, which are dense clusters of integrins bound to the ECM and serve to transmit mechanical information across the cell membrane to the cell's cytoskeleton.

\subsubsection{Fibronectin}

The ECM contains many multifunctional proteins that bind more than one substrate. One of the most commonly studied is a glycoprotein called fibronectin. Fibronectin is involved in many vital cell processes such as cell adhesion, determination of cell morphology, thrombosis, cell migration, and

embryonic differentiation ${ }^{3}$ and contains binding domains for collagen, fibrin, heparin, and cells. ${ }^{4}$ It exists in a dimer structure consisting of two similar subunits, which are cross-linked at their C-terminus ends by disulfide bonds. 
Each subunit has a molecular weight of $\sim 220-250 \mathrm{kDa}^{4}$ and is comprised of serially-repeated modules named type I, type II, and type III. ${ }^{5,6}$ Cells interact with fibronectin through surface receptors called integrins, which bind to the amino acid sequence RGD (arginine-glycine-aspartic acid). While peptides containing the RGD sequence have been shown to bind to integrins and support cellular adhesion, the binding strength of integrins to linear RGD peptides is much reduced in comparison to binding to the native protein. ${ }^{7}$ This indicates that the native fibronectin protein's secondary structure and possible nearby secondary binding domains need to be taken into consideration.

The $\alpha_{5} \beta_{1}$ integrin binds to the RGD primary domain found in the $10^{\text {th }}$ type III $\left(\mathrm{III}_{10}\right)$ repeat and has a synergy-binding domain, PHSRN (proline-histidineserine-arginine-asparagine), found in the $9^{\text {th }}$ type III (III) repeat. ${ }^{3,8}$ The crystal structure of the $\mathrm{III}_{7-10}$ domains of fibronectin show that the RGD binding domain forms a hairpin-like loop, which extends $\sim 10 \AA$ from the surface of the protein and does not have any contact with other regions of the protein ${ }^{4}$ (Figure 1-1). Domains $\mathrm{III}_{9}$ and $\mathrm{II}_{10}$ have very little rotation and tilt between them so fibronectin is almost cylindrical in this region, and the PHSRN and RGD are presented on the same face of the molecule. The two binding domains are separated by $30-40 \AA$. The low amount of protein surface area buried between the $9^{\text {th }}$ and $10^{\text {th }}$ domains indicates that the area is likely to be more flexible than other regions of the protein. ${ }^{4}$ 


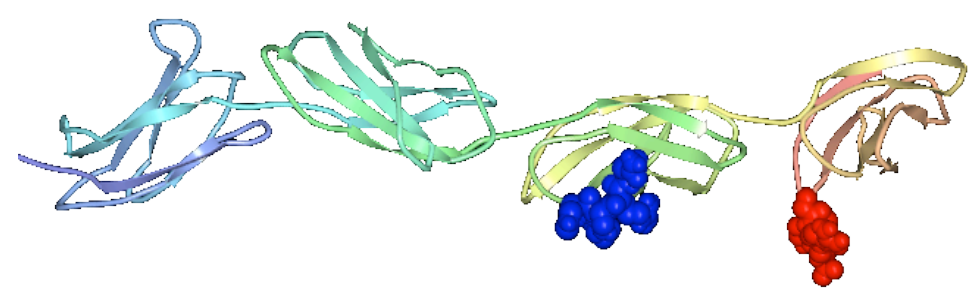

PHSRN RGD

Figure 1-1: Fibronectin $\mathrm{III}_{7-10}$ showing the PHSRN synergy-binding domain and RGD primary binding domain (Adapted from Leahy ${ }^{4}$ )

\subsubsection{Integrins}

Integrins are a family of heterodimeric transmembrane proteins that bind to extracellular matrix proteins. ${ }^{3}$ The binding of integrins to the ECM is substantially weaker than that of hormone receptors and other signaling molecules. Thus integrins typically form multiple weak attachments in a similar fashion to "Velcro." This allows the cells to release cell attachments when needed. They bind to a wide variety of ligands including: fibronectin, laminin, collagen, vitronectin, and fibrinogen. ${ }^{9}$

Integrins are comprised of an $\alpha$ and a $\beta$ subunit, which are non-covalently associated (Figure 1-2). The subunits have very large extracellular domains and very short intracellular domains. The cytoskeleton interacts with the integrin through the $\beta$ subunit's cytoplasmic domain. Eighteen types of subunit $\alpha$ and eight types of subunit $\beta$ have been identified in mammalian cells ${ }^{9}$ (Figure 1-3). These subunits interact to form at least twenty-four different combinations, but not all types are active or even expressed on all cell types. ${ }^{9}$ Knockout experiments of the different subunits produce distinct phenotypes indicating that each integrin has a specific function. ${ }^{9}$ Since more a subunits exist than $\beta$ subunits and integrins containing the same $\beta$ subunits do not necessarily bind the same peptide sequence, the a subunit is likely responsible for the specificity 
of the integrin. ${ }^{10}$ These knockout experiments have produced a range of phenotypes including major developmental defects, perinatal lethality, blockage of preimplantation, reduced leukocyte function, inflammation, hemostasis, and the prevention of angiogenesis and bone remodeling. ${ }^{9}$ Integrins require divalent cations such as $\mathrm{Mn}^{+2}, \mathrm{Mg}^{+2}$, or $\mathrm{Ca}^{+2}$ in order to function, and the type of cation can influence activity and specificity. ${ }^{11}$ The a subunit contains four cationbinding sites, and the $\beta$ subunit contains one cation-binding site. ${ }^{3}$ The binding of divalent cations leads to an "open" or "closed" integrin conformation and thereby regulates ligand binding. ${ }^{9,12}$

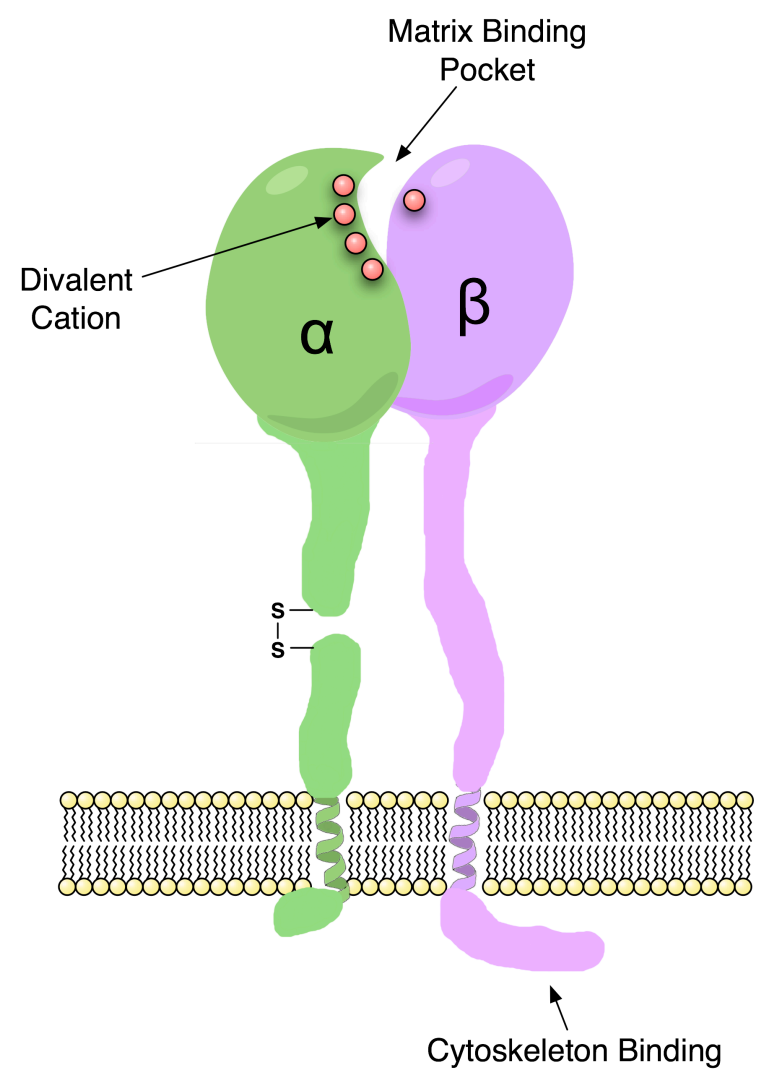

Figure 1-2: Integrin dimer cartoon. Integrins are transmembrane heterodimeric proteins that connect the extracellular matrix and cell's cytoskeleton. (Adapted from Alberts ${ }^{3}$ ) 


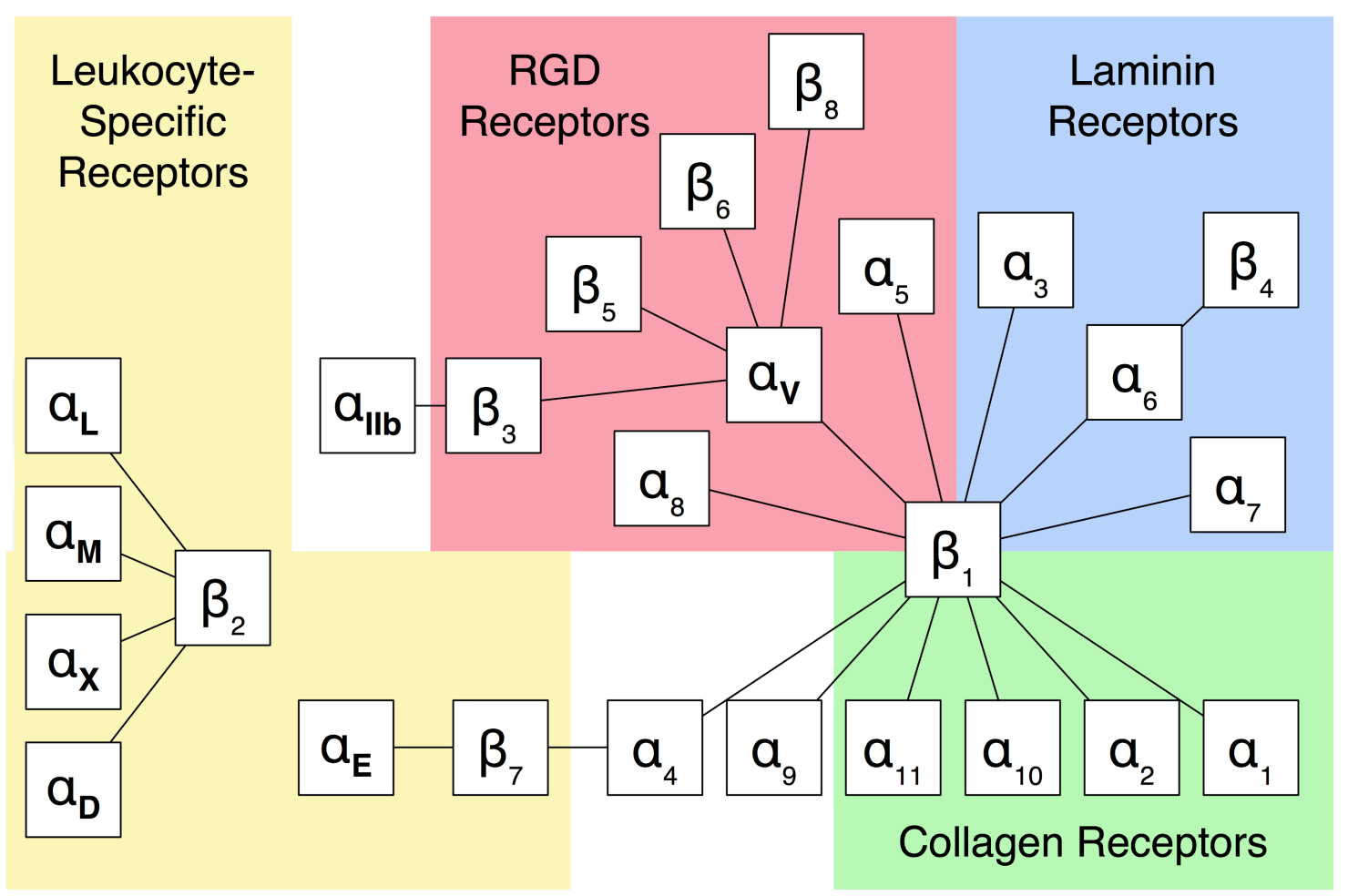

Figure 1-3: Integrin Family (Adapted from Hynes 2002 ${ }^{9}$ )

$a_{5} \beta_{1}$ integrin is one type of integrin and binds to the fibronectin domain $\mathrm{III}_{9-10 .}{ }^{3,8}$ The $\beta$ subunit is primarily responsible for recognizing the RGD peptide when $a_{5} \beta_{1}$ integrin engages fibronectin, and the a subunit must be close by and is primarily responsible for recognizing the PHSRN synergy domain. ${ }^{8,}{ }^{10} a_{5} \beta_{1}$ integrin is important for wound healing, ${ }^{13}$ vascular development, and angiogenesis. ${ }^{14,15}$ During early angiogenesis, $a_{5} \beta_{1}$ integrin is highly expressed on cell surfaces; whereas, other integrins appear to be important for vessel maturation. ${ }^{16}$ Pathological angiogenesis is the rapid growth of tumor-associated blood vessels. These vessels overexpress fibronectin, which contains the binding site for $a_{5} \beta_{1}$ integrin. ${ }^{17}$ Antibodies against $\alpha_{5} \beta_{1}$ integrin have been shown to inhibit angiogenesis in tumors. ${ }^{18} a_{5} \beta_{1}$ integrin has also proven to be vital in developmental angiogenesis. Genetically engineered embryonic mice lacking $a_{5}$ integrin show severe defects in vascular development and show signs of neural crest apoptosis. ${ }^{9,19}$ Studies have shown that $a_{5} \beta_{1}$ integrin aids 
angiogenesis by stimulating endothelial cell proliferation, ${ }^{20}$ promoting endothelial cell migration, ${ }^{21}$ enhancing lumen formation, ${ }^{22}$ and mediating vascular patterning. ${ }^{23}$

\subsection{Cell Adhesion to Engineered Scaffolds}

The scaffold is the structural component of the engineered tissue and provides a mechanism for cell adhesion. Most mammalian cells require anchorage to a substrate. ${ }^{24}$ Binding a soluble protein or peptide sequence is not sufficient for cell viability. ${ }^{25}$ Interactions between a cell and the biomaterial scaffold can be specific or non-specific. Specific interactions are mediated through integrins or other cellular receptors binding to a ligand, which is a specific extracellular protein sequence or molecule that the receptor recognizes. Non-specific interactions include electrostatic interactions, van der Waals forces, and steric repulsions. To encourage cell adhesion in an engineered scaffold, most designs incorporate a bioactive molecule. Methods for immobilization of ligands include using a covalent linker molecule, soft lithography, or protein adsorption. ${ }^{26}$ Designs can either incorporate the entire bioactive molecule or only a certain domain, such as the RGD peptide.

Cellular adhesion to non-modified biomaterials is mediated through protein adsorption from the surrounding media to the biomaterial surface. Protein adsorption is largely dependent upon the topography and free surface energy, which is dependent upon electrostatic, hydrophobic, and van der Waals interactions. $^{26-30}$ Protein adsorption from surrounding media has a significant effect on biocompatibility as some adverse adsorption can trigger the foreign body reaction leading to biomaterial rejection. Many scaffold designs try to actively target cell interaction by including an ECM protein, which helps to regulate cell adhesion, proliferation, growth, differentiation, and migration. ${ }^{31-36}$ Several different methods for incorporating ECM protein moieties have been 
used such as: using a decellularized scaffold, binding whole ECM proteins to a biomaterial, using a protein fragment, and coupling ECM-mimicking peptides to the biomaterial.

Native ECM proteins are easily recognized by cells and have high binding affinities, but they are also macromolecules consisting of many different domains. This gives scaffold designers less control over the orientation of the protein and specificity. Proteins frequently contain secondary binding domains to other molecules, which may lead to undesired cell interactions. Whole proteins can be incorporated either by covalent immobilization or by adsorption. Immobilization chemistry is non-trivial because proteins contain a large number of reactive sites. Since the presentation of the protein cannot be easily controlled, the desired binding site may be obscured. Some researchers have designed protein fragments that contain special functional groups at one terminus to control the orientation of the bound protein fragment. ${ }^{37}$ The use of a fragment also reduces the number of secondary binding sites. Adsorption is another method to incorporate proteins into designs; however, adsorption can lead to a dynamic structural rearrangement or denaturation depending on the substrate, which can alter or destroy protein activity. ${ }^{3,27,28,38}$ Adsorbed proteins also can dissociate from the biomaterial, and once they dissociate they no longer provide biofunctionality to the scaffold. ${ }^{39}$

Peptides have high structural stability because they are much smaller and do not exhibit complex protein folding. Peptides can be engineered to have a high degree of specificity by choosing to present only one binding domain. Their limitation is that peptides do not have the exact same structure as the full protein resulting in lower adhesion than the native protein. ${ }^{40}$ Peptides do, however, allow a high degree of control over the presentation of the binding sequence and the ligand density, both of which are crucial aspects for scaffold 
design. Peptides have been incorporated into a variety of scaffolds including natural polymers, ${ }^{41}$ polyethylene glycol (PEG) hydrogels, ${ }^{42,}{ }^{43}$ and biodegradable copolymers. ${ }^{44-46}$ Peptides have also been used to increase the bioactivity of biomaterials by grafting them to a solid surface $e^{47,48}$ or by creating self-assembling monolayers. ${ }^{49-51}$ Many different biomimetic peptides have been identified by studying the binding domains of native proteins (Table 1-1). The most commonly used in tissue engineering include RGD, PHSRN, IKVAV, and YIGSR. PHSRN and RGD are discussed in further detail in Section 2.2.

Table 1-1: A few biomimetic peptide sequences, the proteins from which they are derived, and cell receptors that they bind

\begin{tabular}{|l|l|l|}
\hline \multicolumn{1}{|c|}{ Peptide Sequence } & \multicolumn{1}{|c|}{ Source Protein } & \multicolumn{1}{c|}{ Receptor } \\
\hline RGD & $\begin{array}{l}\text { Fibronectin, laminin, } \\
\text { collagen }\end{array}$ & Multiple integrins ${ }^{52,53}$ \\
\hline IKVAV & Laminin & $\begin{array}{l}\text { Laminin binding protein } \\
55\end{array}$ \\
\hline YIGSR & Laminin & $a_{1} \beta_{1} \& a_{3} \beta_{1}$ integrin $^{56}$ \\
\hline PHSRN & Fibronectin & $a_{5} \beta_{1}$ integrin $^{8}$ \\
\hline REDV & Fibronectin & $a_{4} \beta_{1}$ integrin $^{51}$ \\
\hline DGEA & Collagen I & $a_{2} \beta_{1}$ integrin $^{57}$ \\
\hline YGYYGDALR & Laminin & $a_{2} \beta_{1}$ integrin $^{58}$ \\
\hline FYFDLR & Collagen IV & $a_{2} \beta_{1}$ integrin $^{58}$ \\
\hline VAPG & Elastase & Elastase receptor \\
\hline KQAGDV & Fibrinogen & $\beta_{3}$ integrins $^{60}$ \\
\hline KRLDGS & Fibrinogen & $a_{M} \beta_{2}$ integrin $^{61}$ \\
\hline
\end{tabular}




\subsection{Other Scaffold Design Considerations}

In addition to requiring adhesion to a substrate for survival, mammalian cells are greatly impacted by the mechanical properties, surface chemistry, rate of degradation, surface patterning, and nanostructure of the scaffold. ${ }^{24}$ The scaffold should encourage cell proliferation, recruit nearby cells to migrate into the tissue, protect the cells from injury, allow for sufficient mass transport, degrade at the same rate that cells produce new tissue, and the degradation products should not be toxic to the cells. ${ }^{62}$ Thus, the most important scaffold design criteria are providing a mechanism for cell adhesion, mechanical properties, bulk structure and shape, porosity, degradation rate, and biocompatibility.

\subsubsection{Mechanical Properties}

Cells are constantly under tension. They pull on the ECM, and the ECM, being a large interconnected protein network, resists deformation and pulls back. Cell tension is maintained by the motor protein, myosin II, found on actin filaments. ${ }^{3}$ Integrin complexes called focal adhesions evolve from smaller focal complexes when an external force is applied to the cell. ${ }^{63}$ In fact, focal adhesion assembly, growth, and maintenance requires mechanotransduction. ${ }^{64} \mathrm{~A}$ decrease in myosin II tension on the cell's cytoskeleton triggers a linear decrease in the focal adhesion size. ${ }^{65}$ Lo et. al. showed that fibroblasts grown on stiffer surfaces have larger focal adhesions than fibroblasts grown on softer surfaces. ${ }^{66}$ Integrin binding to free binding peptides does not lead to focal adhesion formation since the cells are not capable of sustaining tension. ${ }^{25}$ Shear stress has been shown to promote focal adhesion assembly in endothelial cells indicating that the assembly is mediated by external force exerted on the cells. ${ }^{67}$ 
Many studies have examined the effect of the mechanical properties of engineered tissue on the cells. ${ }^{64-66,68,69}$ Mechanical properties can be adjusted by introducing chemical cross-linkers to the polymer scaffold. ${ }^{68}$ Biomaterial surfaces made of the same polymer with different elastic moduli can induce cells to take different differentiation paths. ${ }^{68}$ Cells have been found to migrate from soft to stiffer surfaces, possibly due to a tension gradient in the cytoskeleton created by the substrate's stiffness gradient. ${ }^{66,69}$ Engineered tissues designs should try to mimic the native tissue's mechanical properties. Neural tissue has an elasticity of approximately $0.1 \mathrm{kPa}$, muscle is $\sim 10 \mathrm{kPa}$, and bone is $\sim 100 \mathrm{kPa}^{68}$ The elasticity of the native tissue can be obtained by traditional stress versus strain measurements, but cells view their world on a microscopic level. The mechanical properties of a designed tissue need to consider length and time scales relevant to cells. ${ }^{24}$ Mapping the elastic modulus at the cellular level reveals distinct elastic microenvironments for different types of cells found in the same tissue. ${ }^{24}$

\subsubsection{Scaffold Degradation}

The goal of many tissue-engineering scaffolds is to encourage the cells to actually regenerate the original tissue and create their own scaffold. Thus, the engineered tissue should degrade as the cells to recreate their own matrix. Faster degradation has shown to promote better bone tissue development by encouraging differentiation of stem cells into osteoblasts, ${ }^{70}$ and it also decreased the tissue development time required for a PEG hydrogel. ${ }^{71}$ Mechanisms for biodegradation include: hydrolytic degradation, cell-mediated degradation, or enzymatic degradation. Natural ECM proteins are degraded by proteolysis, which is a type of enzymatic degradation. Proteases secreted by the cells recognize specific sequences in ECM proteins and cleave peptide backbone. Endocytosis is another type of cell-mediated degradation where 
cells engulf extracellular objects. A number of biodegradable polymers, which undergo hydrolytic degradation, have been used in tissue engineering such as poly(lactic acid), poly(glycolic acid), and polycaprolactone. ${ }^{26}$ In order to control the rate of degradation, copolymers are made with different ratios of the homopolymer, which will affect the rate of hydrolysis, ${ }^{72}$ or proteolytic sequences can be chemically incorporated into the scaffold.

\subsubsection{Encouraging Angiogenesis and Cell Migration}

One of the difficulties in tissue engineering is the scale-up required for human implants. ${ }^{26}$ Engineered tissues that perform well in mice do not perform as well in humans because humans are so much larger and require larger tissues. The primary roadblock is the matter of diffusion of nutrients and oxygen to the cells in the center of the tissue. The human body does not rely significantly on diffusion since the penetration length of diffusing molecules in many tissues is limited to $150-200 \mu \mathrm{m}$ from the supplying blood vessel. ${ }^{73-75}$ Transport of oxygen and nutrients is mainly accomplished by the circulatory system. Angiogenesis is the development of new blood vessels from pre-existing blood vessels, and endothelial cell migration is an essential element of angiogenesis. Engineered tissues need to promote angiogenesis to form a vasculature for the tissue. Many tissue designs contain growth factors such as vascular endothelial growth factor (VEGF) to induce angiogenesis. ${ }^{76}$ Another approach is to include adhesion molecules that target integrins involved in angiogenesis.

\subsection{Peptide-Amphiphiles}

An amphiphile is a molecule that is comprised of both hydrophilic and hydrophobic components, and they have been shown to self-assemble into a variety of nanostructures in solution including micelles, vesicles, monolayers, and bilayers. ${ }^{77}$ The term "peptide-amphiphile" can be used to describe any of 
three classes of molecules, which are: 1) true-peptide-amphiphiles made up of only "natural" amino acid residues, 2) peptides which obtain their hydrophobic elements by incorporation of one or multiple long alkyl chains or lipopeptides, and 3) peptide-based block copolymers, which combine synthetic polymers with amino acid residues. ${ }^{78}$ The common traits among all three are that each contains both hydrophilic and hydrophobic parts, and at least part of the molecule is a peptide. Various research efforts are ongoing in all three areas, but this document focuses on the second class, which most researchers simply term "peptide-amphiphiles."

\subsubsection{Amphiphilic Structures}

Amphiphiles self-assemble in aqueous solutions in order to minimize unfavorable entropic interactions between their hydrophobic tails and the surrounding medium. Peptide-amphiphiles have been shown to self-assemble into a wide-variety of nanostructures including micelles, ${ }^{79}$ vesicles, ${ }^{80}$ nanofibers, ${ }^{81}$ monolayers, ${ }^{82}$ bilayers, ${ }^{82}$ nanotubes, ${ }^{83}$ and nanobelts. ${ }^{84}$ One of the most common examples of an amphiphile is soap. Soap molecules form micelles encapsulating the grease particles allowing the grease to dissolve in water and be washed away. Micelles are spheroids that form in aqueous solutions with the hydrophobic tail forming the core of the sphere, and the hydrophilic headgroup oriented outwards to interact with the surrounding solvent (Figure 1-4). Vesicles are also spherical but instead have a doublelayered wall of amphiphiles that encapsulates a hydrophilic core. Micelles are typically much smaller than vesicles. For simple surfactants, micelles have diameters on the order of $5 \mathrm{~nm}$; whereas, vesicles may be $20 \mathrm{~nm}$ to several microns in diameter. ${ }^{85}$ Micelles can also form into cylindrical shapes instead of spherical, and these aggregates are called cylindrical micelles or nanofibers. Monolayers are typically formed on surfaces, and consist of a single layer of the 
amphiphile. Bilayers also typically form on surfaces, but form a double-layer similar to the wall of a vesicle. Monolayers and bilayers can be created using many techniques including Langmuir-Blodgett deposition, chemical adsorption, and micro-contact printing. The molecular shape of the amphiphile has a significant influence on the self-assembly structure. Single hydrocarbon tails typically promote the formation of single-layer structures such as micelles and nanofibers; whereas, double hydrocarbon tails will lead to the formation of bilayers and vesicle structures.

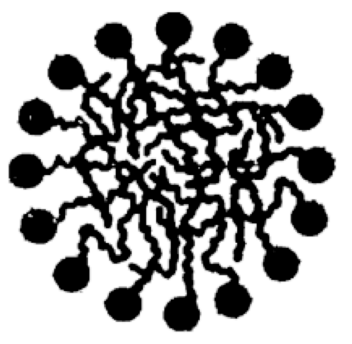

Micelle

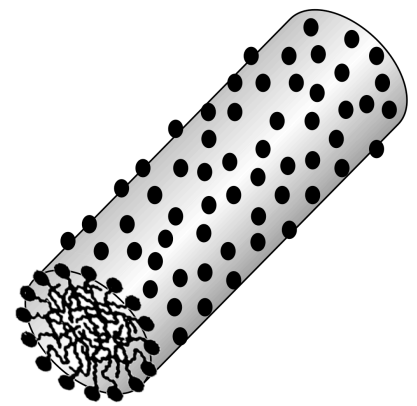

Cylindrical Micelle

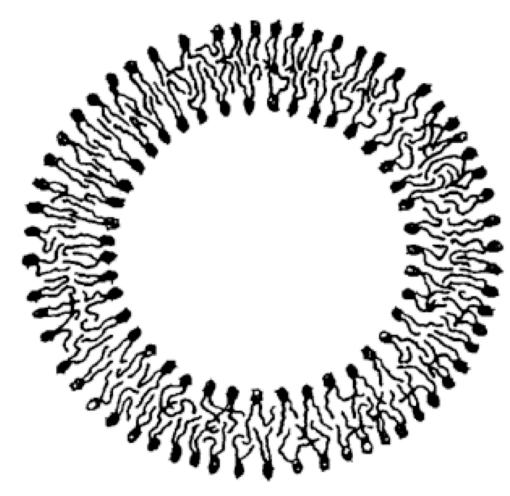

Vesicle

Figure 1-4: Diagram cutaway of a micelle, vesicle, cylindrical micelle, and bilayer. The hydrophobic chains adopt a disordered conformation in the aggregate cores, which are frequently described as liquid-like. (Adapted from Zana ${ }^{85}$ ) 
Peptide-amphiphiles have been widely studied as a biomaterial due to their ability both to self-assemble in solution and ability to interact with biological molecules through their peptide headgroup. The hydrophobic tail not only drives self-assembly, but it has also been shown to influence the secondary structure of the peptide headgroup. For example, collagen type I forms a triple helical structure due to a repeated amino acid sequence Gly-X-Y, where $X$ is frequently proline and $Y$ is a proline-derivative, hydroxyproline. ${ }^{3}$ Research has shown that a collagen mimetic peptide containing a repeated Gly-Pro-Hydroxyproline motif will form a triple helical structure, and the stability of the structure is greatly enhanced by the addition of hydrocarbon tails. ${ }^{86-88}$ The activity of proteins is strongly correlated to the molecular structure, and the ability to control the secondary structure of peptides will allow more effective biomimetic design. The tail can also provide a method for immobilization of peptide-amphiphiles to a surface, and this technique is commonly used in the formation of selfassembled monolayers (SAMs). ${ }^{49,51,89}$ Some researchers have designed a tail with cross-linkable functional groups to lock self-assembled structures into shape..$^{90}$

\subsubsection{Applications for Peptide-Amphiphiles in the Biomedical Field}

Self-assembled peptide-amphiphiles are useful in a variety of biomedical applications including drug delivery, tissue engineering, biomaterial modification, and biosensing. ${ }^{78}$ Vesicles made from lipids, termed liposomes, are widely used for drug delivery applications. Peptide-amphiphiles can be incorporated into liposomes to add bioactive functionality to liposomes. The peptides can interact with the specific receptors and help ensure that the drug is delivered to the target tissue. ${ }^{91}$

One of the most challenging issues in biomaterial design is modifying a surface to control the interactions of cells with the surface. ${ }^{92}$ By controlling specific 
interactions between cells and the surface, the material's biocompatibility can be improved and enhance the function and lifetime of the material. Monolayers and bilayers of peptide-amphiphiles have applications in biosensing, ${ }^{93}$ bioanalytic devices, ${ }^{94}$ and medical implants. ${ }^{95}$ When forming monolayer and bilayer structures of peptide-amphiphiles, researchers can dilute the peptideamphiphiles with molecules such as polyethylene glycol (PEG) to allow more accessibility between cell receptors and the peptide headgroups. ${ }^{49}, 86,96-99$ Introduction of these diluting molecules into the monolayer or bilayer has shown evidence of pattern formation on the surface of the monolayer, which may be useful in biomaterial design that requires surface patterning. ${ }^{99}$ Another example is the use of peptide-amphiphiles to encapsulate carbon nanotubes. ${ }^{100}$ Carbon nanotubes are highly hydrophobic, which imparts low biocompatibility. Any covalently-attached functional group incorporated to increase biocompatibility would affect the electrical properties of the carbon nanotubes. Peptideamphiphiles are able to self-assemble around the carbon nanotubes changing the surface properties without covalent attachment to the carbon nanotubes thus making them biocompatible without compromising their electrical properties. $^{100}$

Peptide-amphiphiles that have a bulky headgroup and a single hydrocarbon tail have an overall conical molecular shape and tend to form nanofibers. Nanofibers show promise as a useful nanostructure for tissue engineering. ${ }^{101}$ Peptide-amphiphile nanofibers can be induced to form self-supporting gels at sufficiently high amphiphile concentrations and when the electrostatic repulsions are overcome by changing the $\mathrm{pH}^{101}$ adding divalent cations, ${ }^{102}$ or by mixing oppositely-charged peptide-amphiphiles. ${ }^{103}$ They have been used as templates to orient hydroxyapatite mineralization, ${ }^{81}$ as scaffolds for cell encapsulation, ${ }^{102}$ and as MRI contrast agents. ${ }^{104}$ Nanofibers are covered in greater detail in Chapter 3. 


\subsection{Organization of Thesis}

The goal of this work is to design a fibronectin mimetic peptide that would selfassemble into nanofibers and form a gel. We focus on mimicking fibronectin because it is one of the primary cell adhesion proteins found in the extracellular matrix. Also, fibronectin binds $a_{5} \beta_{1}$ integrin, which is a vital receptor during wound healing and angiogenesis, both of which are essential in tissue engineering. We approached this task first by designing fibronectin mimetic peptide-amphiphiles for use on $2 \mathrm{D}$ substrates and then expanding the work to 3D scaffold design using peptide-amphiphile nanofibers.

Chapter 2 focuses on the design of fibronectin mimetic peptides to interact with the $a_{5} \beta_{1}$ integrin. Several different fibronectin mimetic peptides were designed to determine the best sequence for cell adhesion. First the synthesis and purification of peptide-amphiphiles is described. Then the different peptideamphiphiles are examined with Langmuir-Blodgett techniques and atomic force microscopy.

Once the best fibronectin mimetic peptide was determined, the challenge was to introduce the peptide into a 3D construct. Peptide-amphiphiles containing a single hydrocarbon tail have been shown to form nanofibers and self-supporting gels, which may be used as scaffolds to support cell growth. Chapter 3 examines peptide-amphiphile nanofibers, and details the design of a fibronectin mimetic peptide-amphiphile that will form a nanofibers and gel for applications in tissue engineering.

Chapter 4 describes the synthesis and purification of the peptide-amphiphile designed for nanofiber formation. This peptide-amphiphile was examined with a lipophilic dye to determine the critical micelle concentration. Cryo-transmission electron microscopy and small angle-neutron scattering were used to determine 
that the peptide-amphiphile did form nanofibers in solution, and rheology measurements showed that at higher concentrations the peptide-amphiphile would form a gel that may be used as a scaffold for tissue engineering.

Chapter 5 provides a summary of the research. 


\section{Chapter 2 Fibronectin Mimetic Peptides}

\subsection{Chapter Overview}

Biomaterials frequently need to encourage cell adhesion, and many designs include a protein or peptide that will interact with cell adhesion proteins. This chapter describes one strategy incorporating one of the most popular biomimetic peptides, RGD, with PHSRN, the synergy-binding domain of fibronectin. Several peptide headgroups were designed to determine the effect of different amino acid sequences separating the binding domains from each other and from the hydrocarbon tail. These fibronectin mimetic peptides were attached to dialkyl tails to make a peptide-amphiphiles. The Langmuir-Blodgett technique was used to transfer the peptide-amphiphiles to mica substrates for atomic force microscopy studies.

\subsection{Biomimetic Peptides RGD and PHSRN}

Fibronectin, as discussed in Section 1.1.1, is one of the most important adhesion proteins in the extracellular matrix. One tactic to encourage cell adhesion is to design a peptide sequence that mimics the adhesion domain of fibronectin. The most common peptide sequence used to encourage cell adhesion is RGD. The RGD adhesion sequence was identified by examining peptide fragments of the cell adhesion domain of fibronectin. ${ }^{52}$ The primary binding domain of fibronectin was identified in the hexapeptide GRGDSP, and RGDS was concluded to be the minimum sequence required for binding. ${ }^{105}$ RGDX has since been found in many other extracellular matrix proteins, where $\mathrm{X}$ may be $\mathrm{S}, \mathrm{V}, \mathrm{T}$, or $\mathrm{A} .{ }^{105}$ The RGD binding domain is very sensitive to slight amino acid variations. The replacement of aspartic acid with glutamic acid $(R G D \rightarrow R G E)$ results in a 100 times reduction in activity. ${ }^{7}$ RGD is recognized 


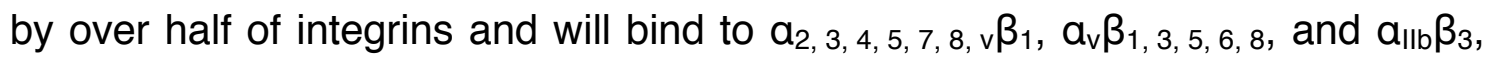
although $a_{2,3,4,7} \beta_{1}$ integrins only bind $R G D$ weakly or under certain conditions. $^{53}$ The spatial conformation of RGD seems to affect its specificity. Looped conformations of RGD tend to be preferred by $a_{v} \beta_{3}$ and $a_{3} \beta_{1}$ integrins; whereas, $a_{5} \beta_{1}$ integrin prefers linear RGD. ${ }^{106}$

While RGD can encourage cell adhesion, the affinity of the tripeptide is relatively low in comparison to the native proteins. The affinity of the hexapeptide GRGDSP binding domain found in fibronectin is approximately 1000 less than that of fibronectin. ${ }^{7}$ The synergy domain in fibronectin, PHSRN, has been shown to increase cell adhesion 100-fold, and binding is specific to the $a_{5} \beta_{1}$ integrin. ${ }^{107,} 108$ This has led researchers to attempt to improve cell adhesion by incorporating both PHSRN and RGD domains into their scaffold designs. Some designs mix the two peptides together on the same surface and have demonstrated that incorporation of both PHSRN and RGD leads to increased $a_{5} \beta_{1}$ integrin binding. ${ }^{97,} 98$ In order to ensure both PHSRN and RGD are able to fit in the integrin-binding pocket, the two sequences need to be available in the proper spatial context and relative orientation. Controlling the presentation of two separate peptides is not an easy task, thus some of the most promising designs are peptides that contain PHSRN and RGD on the same peptide chain.

Most fibronectin mimetic peptide designs contain a flexible linker between the PHSRN and RGD domains in order to mimic the 30-40 $\AA$ distance ${ }^{109}$ between the two domains found in fibronectin. One design attempted to connect the binding domains without a linker (PHSRNRGDS), but it adhered fewer corneal epithelial cells than the two peptides used alone, which indicates that a linker between the two domains is essential. ${ }^{110}$ A poly-glycine linker is frequently chosen because glycine is the smallest amino acid, is the most flexible, ${ }^{111}$ and 
encourages a random peptide conformation due to its slight hydrophobicity. ${ }^{112}$ Kim et. al. showed that for short-term adhesion of osteoblasts, a $G_{6}$ linker shows superior cell attachment over $G_{3}, G_{9}$, and $G_{12}$ linkers. ${ }^{113}$ However, another study shows that a $G_{13}$ linker provides better attachment for macrophages than a $G_{6}$ linker, and that the $G_{6}$ linker actually enhanced the foreign body immune response by encouraging the formation of foreign body giant cells. ${ }^{114,} 115$ The heightened immune response could lead to chronic inflammation and implant rejection. A fully extended $G_{6}$ linker is only $\sim 22 \AA$ long, which is much shorter than the separation found in native fibronectin and may not be long enough to bind $a_{5} \beta_{1}$ integrin properly. Another study has attempted to use a polyethylene glycol (PEG) linker between the PHSRN and RGD domains, and the peptide hybrid did help baby hamster kidney cells spread more than RGD and PHSRN did alone but did not perform nearly as well as native fibronectin. ${ }^{116}$ The PEG linker had an average molecular weight of $3500 \mathrm{Da}$, which fully-extended would be $\sim 300 \AA$ 跑. The PEG linker is not likely to be completely extended in solution; it is likely to be in a coiled state. The root-mean-square end-to-end distance of a $3500 \mathrm{Da}$ PEG melt is $\sim 50 \AA .{ }^{117}$ This indicates that the PEG linker may be too long and may not easily allow the two binding domains to both interact with the integrin-binding site. Some studies have compared the effect of the relative orientation of the PHSRN and RGD binding domains, and they have found that peptide mimetics connecting the domains in the order PHSRN-RGD is more active than mimetics that use the orientation RGD-PHSRN. ${ }^{115}$

\subsection{Design of PR_b}

The previously mentioned studies focused mostly on linking the PHSRN and RGD domains by mimicking the distance between them, but other factors may also be important in accurately mimicking the native fibronectin. Mardilovich 
and Kokkoli designed a new peptide to mimic both the length and the hydrophilicity/hydrophobicity of the amino acids found between the PHSRN and RGD domains. ${ }^{99}$ Analysis of the primary structure of fibronectin indicates that the ratio of hydrophobic to hydrophilic amino acids linking the two binding sites is nearly $1: 1$. In order to preserve the flexible nature of the linker region and also mimic the hydrophilicity/hydrophobicity, a peptide with a linker of alternating serines and glycines was prepared (PR_a, PHSRN(SG) ${ }_{4}$ RGDSP) and coupled to a double-tailed hydrocarbon tail forming a peptide-amphiphile. Of all the amino acids, glycine has the smallest hydrophobic side chain and serine has the smallest hydrophilic side chain. Serine and glycine are also the two most flexible amino acids. ${ }^{111}$ Table 2-1 details the different peptide sequences and the naming convention used in this work.

Table 2-1: Names and peptide sequences used in this study

\begin{tabular}{|l|l|}
\hline \multicolumn{1}{|c|}{ Name } & \multicolumn{1}{c|}{ Peptide Sequence } \\
\hline PR_a & PHSRN(SG) $)_{4}$ RGDSP \\
\hline PR_b & KSSPHSRN(SG) ${ }_{5}$ RGDSP \\
\hline PR_c & KSSPHSRN(G) ${ }_{10}$ RGDSP \\
\hline PR_d & KSSPHSRN(S) ${ }_{10}$ RGDSP \\
\hline PR_e & PHSRN(SG) $)_{5} R G D S P$ \\
\hline PR_f & KSSSSSPHSRN(SG) $)_{5}$ RGDSP \\
\hline
\end{tabular}

The tail was attached to the N-terminal end of the peptide because the chemistry is easier than attaching the tail to the $\mathrm{C}$-terminal end. Also, previous studies show that the PHSRN-RGD conformation is more effective, and putting the tail on the $\mathrm{N}$-terminal end ensures that the primary domain is on the outside and allows easier access to integrin binding. The amphiphile was deposited on 
a hydrophobized mica substrate for atomic force microscopy (AFM) adhesion studies. $a_{5} \beta_{1}$ integrin immobilized on the AFM tip showed that the integrins adhered to the PR_a surface with approximately the same affinity as they bound to a surface coated with GRGDSP peptide-amphiphile. ${ }^{99}$

The $(\mathrm{SG})_{4}$ linker has a theoretical length of $29.6 \AA$, assuming $3.7 \AA$ per amino acid, ${ }^{98,118}$ which may be too short to allow for optimal integrin binding. A new peptide-amphiphile, PR_b (KSSPHSRN(SG) $)_{5}$ RDSP), was designed to have an increased linker length of $37 \AA$ and a KSS spacer to extend the PHSRN domain from the hydrophobic tails ${ }^{119}$ (Figure 2-1). PR_b coated substrates were found to adhere human umbilical vein endothelial cells (HUVEC) equally as well as a fibronectin-coated substrate and performed significantly better than PR_a and GRGDSP. ${ }^{119}$ Integrin-blocking assays show that HUVEC adhesion to PR_b is mediated by $a_{5} \beta_{1}$ integrin. ${ }^{119}$

\section{Spacer Linker}

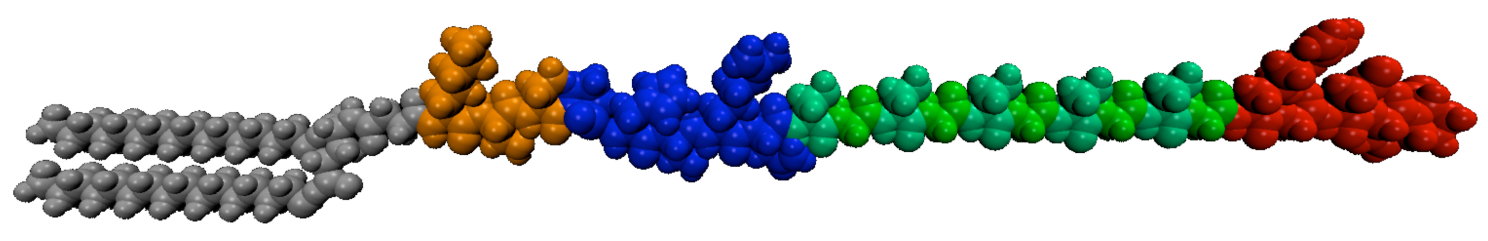

\section{Dialkyl Tail - KSSPHSRNSGSGSGSGSGRGDSP}

Figure 2-1: PR_b fibronectin mimetic peptide-amphiphile structure. A dialkyl $C_{16}$ tail is coupled to a KSS spacer, PHSRN synergy-binding domain, $(\mathrm{SG})_{5}$ linker, and RGDSP primary binding domain.

The previous study indicates that PR_b is an excellent fibronectin mimetic peptide. The next step was to determine whether the hydrophilicity/hydrophobicity of the serine/glycine linker, the KSS spacer, or both had an impact on the efficacy of PR_b. Several more peptides were designed to be tested with AFM and cell adhesion studies. PR_c and PR_d 
tested the effect of linker hydrophilicity and contained $G_{10}$ and $S_{10}$ linkers, respectively. PR_e and PR_f probed the effect of the spacer. PR_e, like PR_a, contained no spacer, and PR_f contained an elongated KSSSSS spacer. The peptides were coupled to dialkyl tails and deposited on hydrophobized mica substrates using the Langmuir-Blodgett technique for further study with AFM and cell adhesion studies.

\subsection{Peptide-Amphiphile Synthesis}

Peptide-amphiphiles were synthesized using solid-phase peptide synthesis. Solid-phase peptide synthesis has a distinct advantage over solution phase chemistry because the synthesis is carried out with the peptide covalentlyattached to small beads, which allows for easy separation of the product from reactants by filtration. The peptide synthesis was performed by the Peptide Synthesis Facility at the University of Minnesota and carried out in step-wise fashion in which only one amino acid was added to the peptide during each coupling reaction. After the peptide synthesis was complete, the peptide was coupled to a dialkyl hydrocarbon tail. Tails were synthesized via a two-step synthesis. The peptide-amphiphile was cleaved from the bead and purified as described in detail below.

\subsubsection{Synthesis of Dialkyl Tail}

The hydrophobic tail of the peptide-amphiphile was composed of two hydrocarbon chains linked by glutamic acid and coupled to a $C_{2}$ spacer. The synthesis was performed as described by Berndt et. al. ${ }^{82}$ The reaction scheme is shown in Figure 2-2. In the first step, hexadecanol was coupled to glutamic acid by acid-catalyzed condensation with para-toluenesulfonic acid monohydrate $(\mathrm{TsOH})$ in toluene. The reaction proceeded at $120-135^{\circ} \mathrm{C}$ until completion. Toluene was removed by rotary evaporator and acetone was 
added to the product. The acetone solution was warmed until the solids dissolved and allowed to cool in the fridge overnight. The $\mathrm{TsOH}$ salt of the dialkyl ester crystallized in the cooled acetone and was filtered from the solution. Dissolution in acetone and recrystallization was repeated several times to purify the dialkyl ester prior to the addition of the $\mathrm{C}_{2}$ spacer.

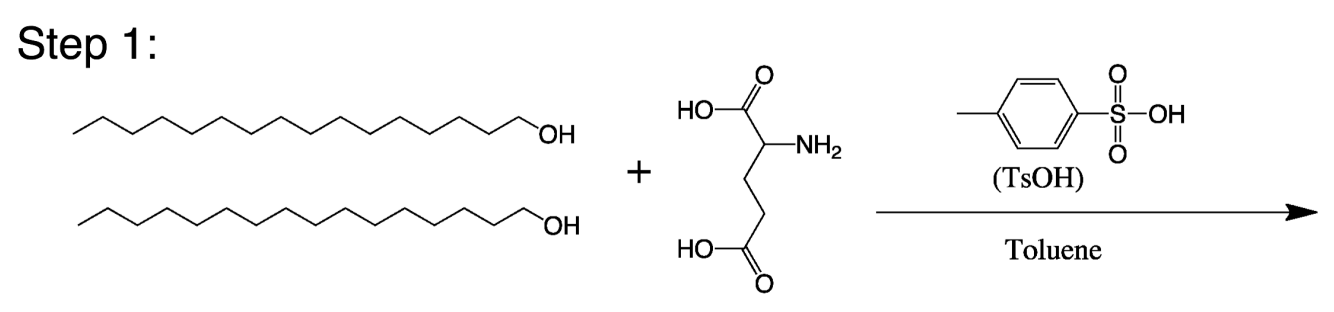

\section{Step 2:}
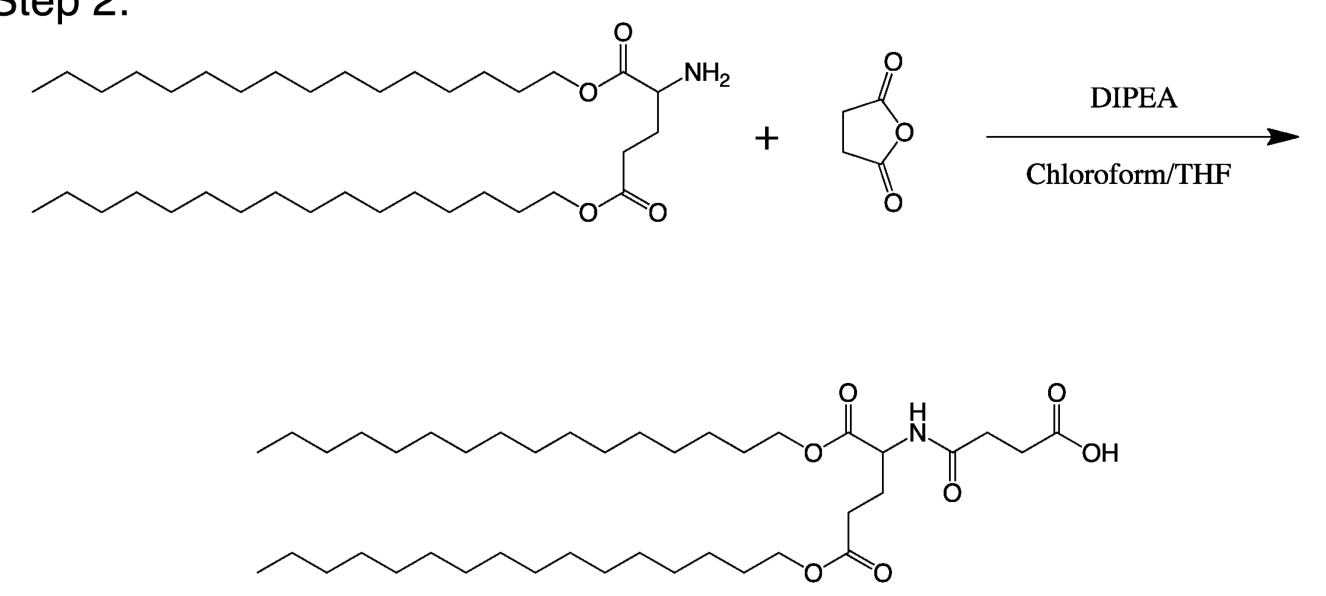

\section{Dialkyl Tail}

Figure 2-2: Dialkyl tail synthesis. Step 1 shows the coupling of hexadecanol tails to glutamic acid by $\mathrm{TsOH}$ acid-catalyzed condensation. Step 2 shows the coupling of the free amino group to succinic anhydride to form the completed dialkyl tail.

In the second step of the synthesis, the free amino group was coupled to succinic anhydride in an equimolar solution of chloroform and tetrahydrofuran (THF) in the presence of diisopropylethylamine (DIPEA) at $40-50^{\circ} \mathrm{C}$. The reaction was allowed to proceed for 2 hours. The solvent was removed by 
rotary evaporation. The product was redissolved in ethyl acetate, cooled in the refrigerator until crystallization, and dried in a vacuum oven. The structure was verified using proton nuclear magnetic resonance (NMR) spectroscopy by other members of the Kokkoli group.

\subsubsection{Solid Phase Peptide-Amphiphile Synthesis}

The peptide headgroup synthesis was performed using standard fluorenylmethoxycarbonyl (Fmoc) solid peptide synthesis by the Oligonucleotide \& Peptide Synthesis Facility at the University of Minnesota. The fibronectinmimetic peptide-amphiphiles used in this study were all prepared as peptide amides, which means that the C-terminus of the peptide headgroup was an amide instead of the conventional acid group. All figures in this section reflect the use of peptide amides. In Fmoc solid phase peptide synthesis amino acids to be added to the peptide are protected on their $\mathrm{N}$-terminus by the base-labile Fmoc-protecting group. This prevents more than one amino acid from coupling to the peptide at each coupling step. The side chains of amino acids are also protected by various acid-labile groups to prevent the unwanted coupling to a side chain. The side chain protecting groups are removed during the cleavage step at the end of the synthesis. The Fmoc-protecting group was removed from the synthesized peptide chain in a solution of $20 \%$ piperidine in dimethylformamide (DMF). The beads were deprotected for 20 minutes with mild agitation, rinsed, and the deprotection step was repeated to ensure complete deprotection.

Peptide-amphiphiles are created by attaching a hydrocarbon tail to the hydrophilic peptide headgroup. The synthesized dialkyl tail used in this study contained a carboxylic acid end group, which was coupled to the amino group on the N-terminus of the peptide using the same chemistry as the amino acid addition (Figure 2-3). The prepared hydrocarbon tails were coupled to the 
growing peptide chain using three times excess of the Fmoc-protected amino acid, three times excess $O$-(benzotriazole-1-yl)- $N, N, N^{\prime}, N^{\prime \prime}$-tetramethyluronium hexofluorophosphate (HBTU), and six times excess DIPEA in DMF. HBTU binds the $\mathrm{COOH}$ end of the amino acid and helps it react with the free primary amine on the end of the peptide chain. The coupling reaction was allowed to proceed at room temperature under mild agitation for 2-4 hours. If the solution formed a gel, dichloromethane (DCM) was added to aid the solubility of the tails. A few beads were removed for testing by the Kaiser test to ensure complete coupling. The Kaiser test uses solutions of ninhydrin, phenol, and potassium cyanide to test for the presence of free primary amines. ${ }^{120}$ A positive test indicates that the coupling was not complete, and the reaction was repeated. If the Kaiser test reveals the beads contain no free primary amines, coupling was complete. If proline, which has a secondary terminal amine, is the amino acid on the $\mathrm{N}$-terminus (as is the case for PR_a and PR_e), the chloranil test was used. ${ }^{121}$ 

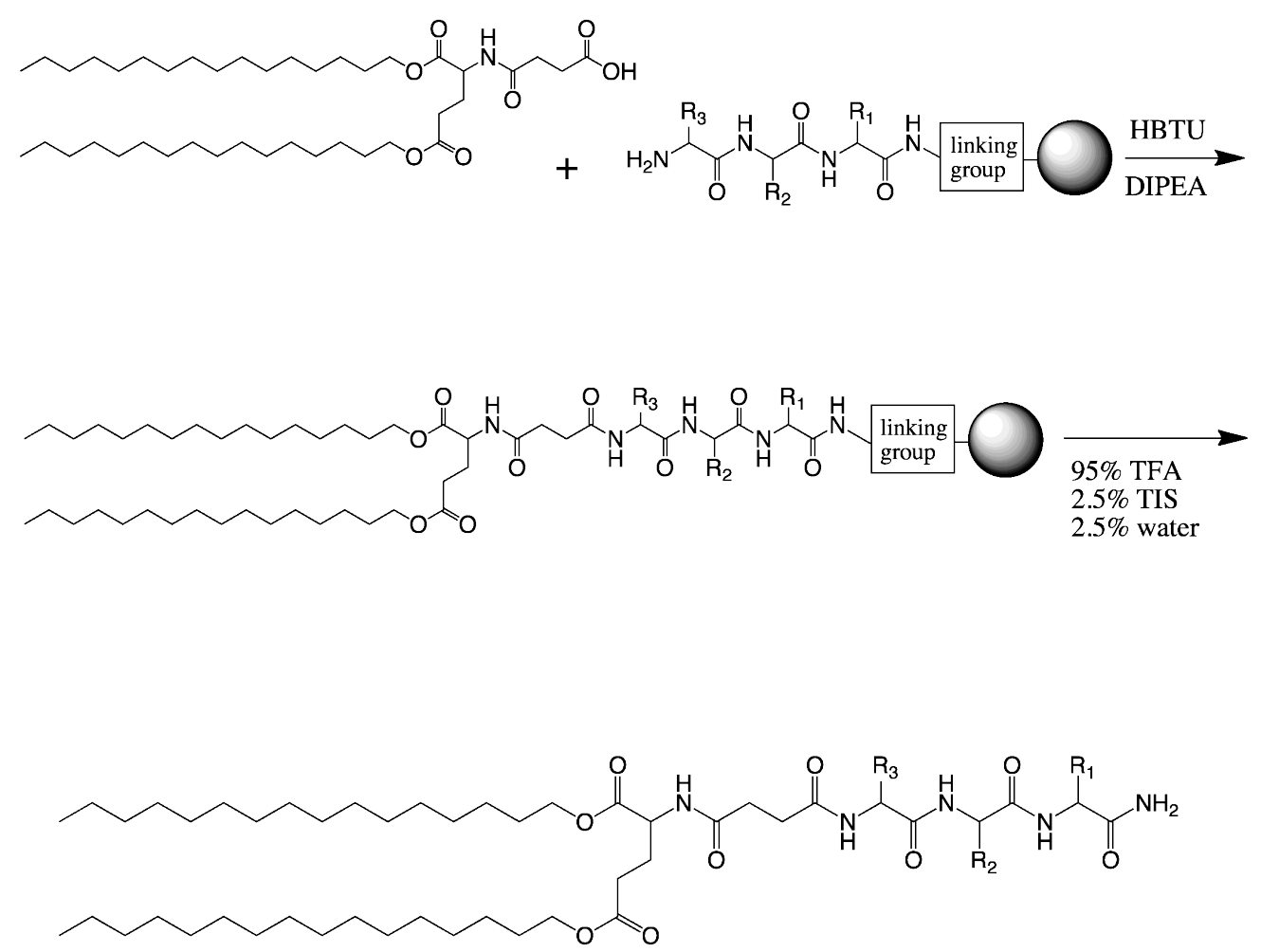

Figure 2-3: Coupling hydrocarbon tail and cleaving peptide-amphiphile from resin bead. First, the hydrocarbon tail is coupled in a similar mechanism as amino acid coupling. Peptide shown is an example tripeptide with side groups $R_{1}, R_{2}$, and $R_{3}$. In the second step, cleavage from the bead occurs in a solution with a high concentration of trifluoroacetic acid (TFA).

\subsubsection{Cleaving from the Solid Support}

Peptides and peptide-amphiphiles are cleaved from the bead in a strong solution of trifluoroacetic acid (TFA) (Figure 2-3). TFA both cleaves the peptideamphiphile from the bead and removes the protecting groups on the amino acid side chains. The fibronectin mimetic peptide-amphiphiles were grown on a PAL resin, which results in a C-terminal amide after cleavage. The peptideamphiphiles were cleaved in a solution of $95 \%$ TFA, $2.5 \%$ triisopropylsilane (TIS), and $2.5 \%$ water for 2 hours.

After cleaving the fibronectin mimetic peptide-amphiphiles from the solid support, the solution was filtered to remove the spent beads from the 
amphiphile solution. The beads were rinsed with excess TFA to maximize product recovery. The TFA-product solution was concentrated using a rotary evaporator. Next, 10-20 times excess of cold diethyl ether was added to precipitate the peptide-amphiphile while leaving impurities in solution. The ether mixture was centrifuged at $4000 \mathrm{rpm}$ for 5 minutes, the pellet was redissolved in a small amount of DMF, and the precipitation process was repeated. The resulting crude peptide-amphiphile was dried under an air stream, redissolved in water, and lyophilized.

\subsubsection{Purification of Crude Product}

The crude peptide-amphiphile was purified using preparative reversed-phase high-pressure liquid chromatography (HPLC). HPLC is a form of column chromatography that is frequently used in biochemistry laboratories to separate, identify, and quantify compounds. ${ }^{122}$ A mobile phase carries the sample through the column containing a stationary phase, which retains the different compounds in the sample according to their chemical makeup resulting in the separation of the different compounds. Reversed-phase HPLC columns have a hydrophobic stationary phase, and the mobile phase is typically a mixture of water and an organic solvent such as acetonitrile $(\mathrm{ACN})$, methanol $(\mathrm{MeOH})$, or isopropanol (IPA). Molecules are retained in the column by hydrophobic interactions with the stationary phase. Stationary phases are typically made of silica particles, which have been modified with an alkyl group. Typical alkyl groups are $\mathrm{C}_{18} \mathrm{H}_{37}, \mathrm{C}_{8} \mathrm{H}_{17}$, and $\mathrm{C}_{4} \mathrm{H}_{9}$. Longer alkyl chains increase the retention time of non-polar molecules. Retention time also varies with the composition of the mobile phase. Increasing the organic component of the mobile phase results in a shorter retention time of the hydrophobic components by reducing the hydrophobic interactions between the stationary phase and the analytes. 
All peptide-amphiphiles used in this study were purified using a mobile phase gradient of $0.1 \%$ TFA in water (mobile phase A) and $90 \%$ ACN: $10 \%$ IPA (mobile phase B). The gradient used is shown in Table 2-2. The gradient starts at $30 \%$ of the organic phase $(\mathrm{B})$ and increased at a rate of $1 \% \mathrm{~B} / \mathrm{min}$ for 60 minutes. Then the gradient was run isocratically for 10 minutes to ensure all of the strong hydrophobic molecules elute, and then the column was restored to its starting mobile phase to prepare for another run. The stationary phase was a $C_{4}$ column and was chosen for the peptide-amphiphile purification to reduce the retention time because the double tail is sufficiently hydrophobic that it interacts strongly with columns modified with longer alkyl groups.

Table 2-2: HPLC gradient used in purification of dialkyl peptide-amphiphiles. Mobile phase $B$ is 90:10 ACN:IPA.

\begin{tabular}{|c|c|}
\hline Time (min) & \% B \\
\hline 0 & 30 \\
\hline 60 & 90 \\
\hline 70 & 90 \\
\hline 71 & 30 \\
\hline 85 & 30 \\
\hline
\end{tabular}

The synthesis and purification of the fibronectin mimetic peptide-amphiphiles was verified by matrix-assisted laser desorption/ionization time of flight (MALDI) mass spectroscopy. MALDI is a soft ionization technique, which allows for the analysis of large biomolecules that would be fragmented by ionization used in conventional mass spectroscopy. A matrix is combined with the sample to absorb the laser light and assist with the vaporization and ionization of the sample. 
Samples were analyzed using a Bruker Reflex III system. For this study, a saturated solution of a-cyano-4-hydroxycinnamic acid matrix was prepared in $1: 1$ mixture of $A C N$ and water and $0.1 \%$ TFA. Samples for MALDI analysis were prepared mixing $2 \mu \mathrm{L}$ of sample with $10 \mu \mathrm{L}$ of saturated matrix solution. One $\mu \mathrm{L}$ of the sample-matrix mixture was spotted onto the MALDI plate and allowed to dry completely. MALDI spectra were obtained using the reflectron mode. An example spectrum of purified PR_b peptide-amphiphile is shown in Figure 2-4. The $x$-axis is the mass-to-charge ratio $(\mathrm{m} / \mathrm{z})$. The theoretical mass of PR_b peptide-amphiphile is $2821.4 \mathrm{Da}$, so the $[\mathrm{M}+\mathrm{H}]^{+}$peak is expected at 2822.4 Da. The MALDI spectrum's primary peak is $2823.0 \mathrm{Da}$, which indicates that the synthesis was successful.

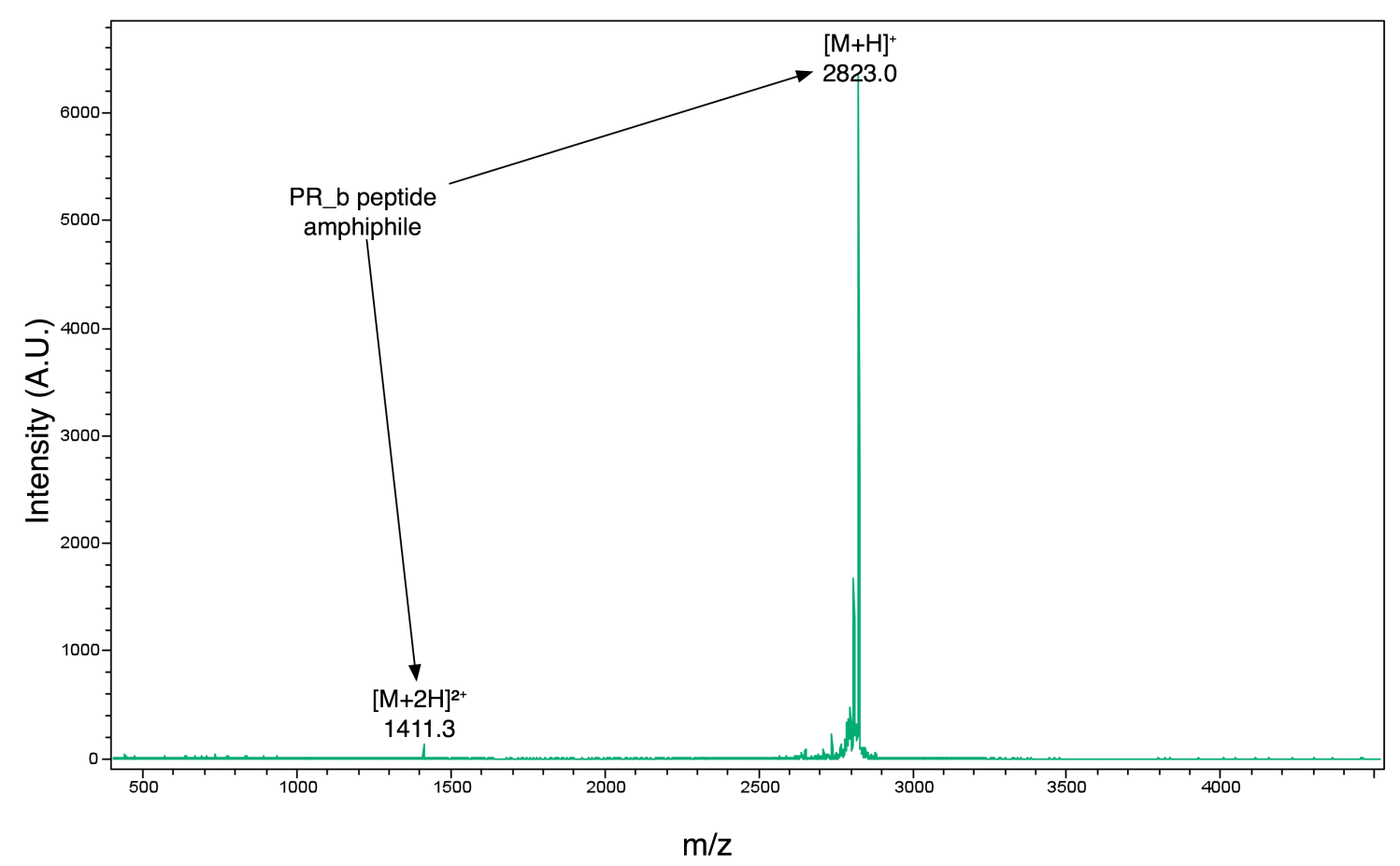

Figure 2-4: MALDI spectrum of purified PR_b peptide-amphiphile. $\mathrm{m} / \mathrm{z}$ is the mass-to-charge ratio read by the detector. The spectrum shows both $a+1$ and +2 charge state for PR_b and verifies the PR_b peptide-amphiphile synthesis. 
MALDI was performed on all the major HPLC peaks to identify the peak that contained pure peptide-amphiphile. The peak was collected, and the solution was dried under air, resolvated in Milli- $Q$ water, and lyophilized. The purified peptide-amphiphile was stored at $-20^{\circ} \mathrm{C}$ until needed.

\subsection{Langmuir Blodgett Technique}

The Langmuir-Blodgett (LB) technique is a method of preparing thin films on a substrate. Thin films formed by lipophilic and amphiphilic molecules at air-liquid interfaces have been known since the 1700's. In 1774, Benjamin Franklin described pouring a teaspoon of oil on a pond and watching as the film covered an area of about half an acre. ${ }^{123}$ Over a hundred years later in 1890, Lord Rayleigh repeated Franklin's experiment and calculated that the resulting thin film was $2 \mathrm{~nm}$ thick and hypothesized that the film was the thickness of one molecule. $^{124}$ In 1891, a German woman, Agnes Pockles, developed a rudimentary surface balance by floating a button in her kitchen sink to measure the surface tension difference as a function of surface area available to different oils. $^{125,126}$ Irving Langmuir studied fatty acid, ester, and alcohol monolayers floating on water in the 1910's and 1920's. ${ }^{127}$ He designed the device now called a Langmuir trough to spread his molecules on the liquid interface. He confirmed that the molecules formed films one molecule thick. He also determined that in order to minimize free energy, amphiphiles self-assemble on the air-liquid interface with their hydrophilic heads in the water and their hydrophobic tails in the air. Katherine Blodgett furthered Langmuir's techniques by detailing how to transfer sequential monolayers onto substrates. ${ }^{125,} 128$

In any liquid, the molecules experience an attractive force from the surrounding molecules. In the bulk of the liquid, these forces are balanced in all directions due to the isotropic nature of the surroundings. However, at the interface the molecules are not surrounded on all sides by other liquid molecules, leading to 
a force imbalance at the air-liquid interface and an overall net attractive force toward the bulk causing the air-liquid interface to spontaneously minimize its area. $^{125,129}$ This excess free energy at the surface is frequently described as surface tension. Polar solvents have strong intermolecular interactions, which results in high surface tensions. The surface tension of pure water is $72.8 \mathrm{mN} / \mathrm{m}$ at room temperature and is the highest surface tension of the nonmetallic liquids. The high surface tension makes water a very good subphase for Langmuir film experiments. ${ }^{130}$

A typical Langmuir-Blodgett apparatus has a trough in which a subphase, usually water, is poured (Figure 2-5). The trough is made of a hydrophobic substance such as Teflon. Two hydrophilic barriers rest on top of the trough in contact with the subphase and are used to compress the surface of the subphase to constrict the interfacial area available to the monolayer. Amphiphilic molecules can be spread on the water surface in between the two barriers by first dissolving the molecules in a volatile hydrophobic solvent and then gently spreading drops of the solution on the water surface. The volatile solvent will evaporate and the amphiphilic molecules will be left behind as a monolayer. When a monolayer is spread on a water surface, the surface tension decreases.

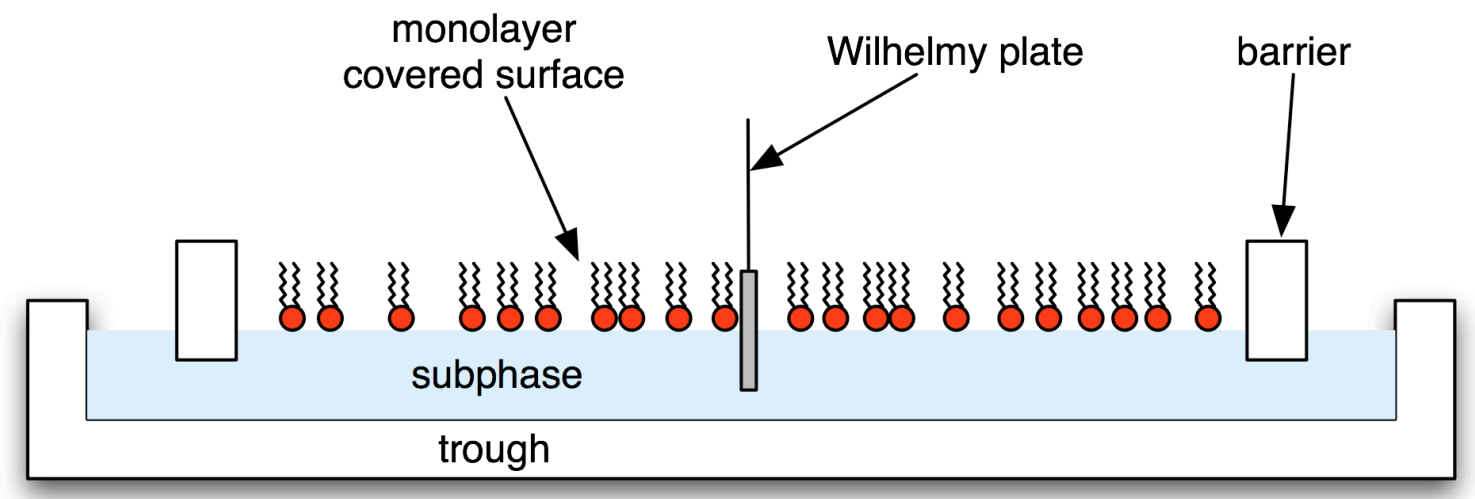

Figure 2-5: Langmuir-Blodgett trough schematic 
Surface tension changes are measured by a very thin piece of platinum, called a Wilhelmy plate, that is partially immersed in the water. The surface pressure is monitored by measuring the changes in weight of the plate according to Equation 2-1. ${ }^{125,129}$ The force, $F$, on a plate of dimensions $l_{p}, w_{p}$, and $t_{p}$ immersed to a depth of $h_{l}$, is dependent upon the density of the plate, $\rho_{p}$, the density of the liquid, $\rho_{l}$, the acceleration due to gravity, $g$, the liquid surface tension, $\gamma$, and the contact angle of the liquid and plate, $\theta$.

Equation 2-1: Force balance on Wilhelmy plate

$F=\rho_{p} g l_{p} w_{p} t_{p}+2 \gamma\left(t_{p} w_{p}\right)(\cos \theta)-\rho_{l} g t_{p} w_{p} h_{l}$

For a very thin plate, $w_{p} \gg t_{p}$, that is wetted completely $\left(\theta=0^{\circ}\right)$ the equation reduces to Equation 2-2. The surface pressure, $\Pi$, can be calculated directly from the change in weight $(\Delta F)$ of the Wilhelmy plate.

Equation 2-2: Surface pressure as a function of the change in weight of the plate and the width of the plate

$\Pi=\frac{\Delta F}{2 w_{p}}$

\subsubsection{Langmuir Monolayer Isotherms}

Monolayer properties are determined by measuring the surface pressure as a function of the water-surface area available to the monolayer. Figure 2-6 shows a surface pressure-area isotherm of an idealized monolayer. Isotherms are obtained by measuring the surface pressure of a monolayer while compressing the film at a constant rate. Figure 2-6 shows several distinct regions corresponding to different phases of the monolayer. In the "gas phase," the molecules have a large area available to them allowing them to spread out, and they do not interact much. When the monolayer is compressed, the monolayer undergoes a transition to the "liquid expanded" phase where the molecules start 
to interact more. This transition is usually marked by a plateau on the isotherm, indicating a coexistence of the gas and liquid expanded phases. As the molecules begin to interact in the liquid expanded phase, the hydrocarbon tails start to "lift off" the air-water interface. ${ }^{131}$ The film and hydrocarbon chains in the liquid expanded phase are mostly disordered. ${ }^{132}$ Upon further compression, the monolayer enters a "liquid condensed" phase; wherein, the hydrocarbon chains are highly extended, and the film exhibits some long-range packing order. With further compression, the film enters a "solid" phase. The packing in the solid phase is very tight similar to packing seen in crystals. Compression beyond the solid phase leads to monolayer collapse where the monolayer becomes unstable and the amphiphiles start to pack on top of each other in a $3 \mathrm{D}$ structure. The collapse appears as either a horizontal plateau or a rapid decrease in the surface pressure. Depending on the length of the hydrophobic region and the chemical nature of the headgroup, the isotherm can exhibit some or all of these phases, and it can have transitions between the phases or no transitions at all. ${ }^{125,133}$ 


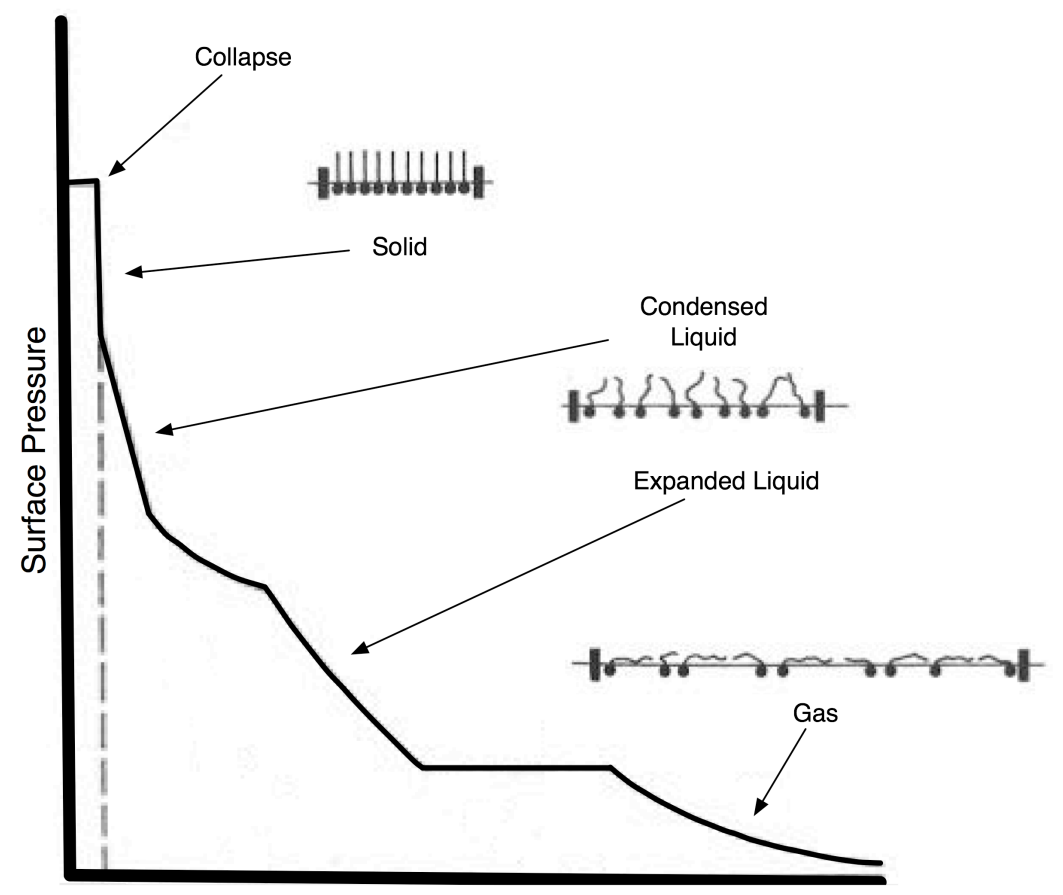

Surface Area available to monolayer

Figure 2-6: Example pressure-area isotherm for a typical surfactant with labeled domains. The side figures depict the morphological changes in the amphiphile as the molecules are compressed.

(Reproduced from KSV LB Manual) ${ }^{134}$

\subsubsection{LB Isotherms of Fibronectin Mimetic Peptide-Amphiphiles}

Peptide-amphiphiles were dissolved in chloroform at a concentration of $1 \mathrm{mg} / \mathrm{mL}$. The solutions were stored in the fridge and warmed to room temperature prior to use. If the solution was cloudy, it was heated in a $50^{\circ} \mathrm{C}$ water bath until it was clear. Isotherms of the fibronectin mimetic peptideamphiphiles were obtained using a KSV 5000 LB system (KSV Instruments, Helsinki, Finland). The trough was cleaned prior to use with chloroform and methanol to remove any contamination. The hydrophilic barriers were cleaned with methanol or ethanol. Next, the subphase, autoclaved Milli-Q water was added to the trough, and the surface was cleaned by vacuum suction. The system cleanliness was determined by compressing the barriers completely and monitoring the surface pressure. The water surface was determined to be free 
of contamination if the change in surface pressure was less than $0.2 \mathrm{mN} / \mathrm{m}$. Approximately $100 \mu \mathrm{L}$ of $1 \mathrm{mg} / \mathrm{mL}$ peptide-amphiphile solution was spread on the air-water interface. The chloroform was allowed to evaporate for 10 minutes. The barriers were compressed at a rate of change relative to the remaining area, 5 area\%/min.

Pressure-surface area isotherms were measured to examine the different peptide-amphiphile intermolecular interactions (Figure 2-7). The collapse pressure for all the molecules occurred between $61-71 \mathrm{mN} / \mathrm{m}$. At the collapse point, the monolayer becomes unstable, and the molecules begin forming threedimensional multilayers. PR_a, PR_b, PR_c, and PR_e all exhibit similar behaviors in that they all have similar gas and liquid expanded phases and then undergo a transition between $35-45 \mathrm{mN} / \mathrm{m}$ to the liquid condensed phase. The transition is most evident in PR_a and PR_e, but slight humps can be seen in the isotherms for PR_b and PR_c. PR_f does exhibit a phase transition but at a significantly lower surface pressure of $18.5 \mathrm{mN} / \mathrm{m}$. PR_d behaves much differently than the other peptide-amphiphiles and does not display a secondary phase transition. At low molecular areas, PR_d and PR_f show a similar isotherm, namely, they both do not start exerting high surface pressures until the molecules are pressed much closer together. This is likely due to the greater number of serine residues in the PR_d and PR_f peptides. PR_d has 10 serines between the PHSRN and RGD domains, and PR_f has 5 serines in a row before the PHSRN domain. Serine has a small hydroxyl side chain capable of forming hydrogen bonds. Interactions between the peptide-amphiphiles through hydrogen bonds may encourage close association between the molecules. In PR_d, this results in the lack of the secondary transition, and in PR_f, the secondary transition occurs at a much lower surface pressure. 


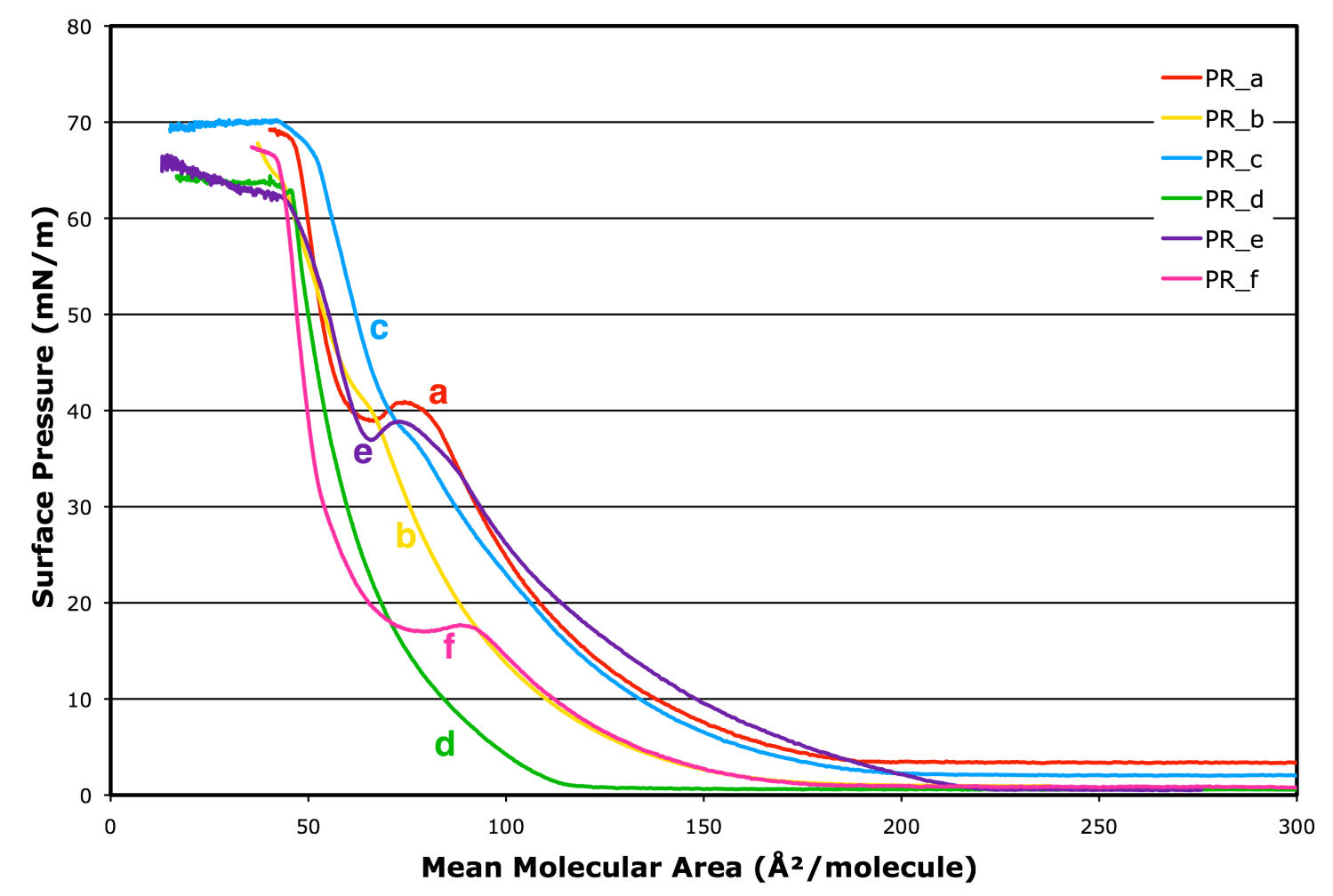

Figure 2-7: $L B$ isotherms of PR_a-PR_f

\subsubsection{Langmuir-Blodgett Deposition}

For further studies, the monolayer can be transferred onto a surface, by dipping a substrate through the air-water interface, creating a Langmuir-Blodgett (LB) film $^{125}$ (Figure 2-8). To build up a multilayer of amphiphiles, the substrate can be dipped many times. The substrate is oriented vertically so the surfaces that are to be coated are perpendicular to the air-water interface. A sample pulled upwards from the liquid to the air will result in a deposited layer of the hydrophilic headgroups oriented toward the substrate and the tails oriented outwards. A sample lowered from the air to the liquid will create a deposited layer with the tails oriented toward the substrate and the headgroups oriented outwards. When the substrate is hydrophilic, the first layer should be deposited so the hydrophilic heads adsorb to the substrate. When the substrate is 
hydrophobic, the first layer is transferred so that the tails are oriented toward the substrate. Mica is commonly used as a substrate because it can be cleaved to create an atomically flat surface. The most common type of LB film deposition is type Y; wherein, a substrate is first pulled up through an LB film, and a second layer is deposited on a downstroke so that the hydrophilic headgroups are oriented outwards. ${ }^{125}$ This creates a basic bilayer structure, and is illustrated in Figure 2-8.

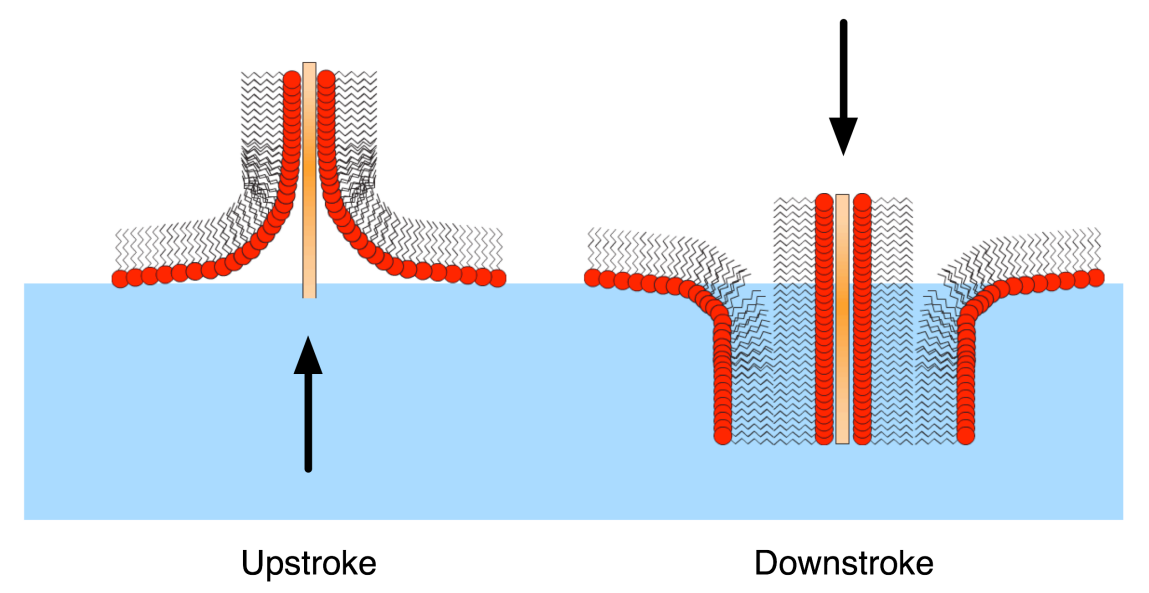

Figure 2-8: Type Y LB deposition. On the upstroke, molecules are deposited with hydrophilic heads toward the substrate. On the downstroke, the tails face the substrate and a bilayers structure is formed.

Deposition is usually performed in the "solid" or "liquid-condensed" phases where the surface pressure is high enough to ensure a uniform, homogenous transfer. A feedback control loop monitors the surface pressure during deposition and adjusts the barriers to ensure a constant surface pressure. The quality of the deposited layers is characterized with a parameter called the transfer ratio, $\tau$. The transfer ratio is defined as the ratio between the decrease in monolayer area on the air-liquid interface, $A_{l}$, and the area of the substrate, $A_{s}$ (Equation 2-3). The decrease in monolayer area is measured by the distance that the barriers have to move to maintain a constant surface pressure. The ideal transfer ratio is $\tau=1$. 
Equation 2-3: Transfer ratio for supported LB films

$\tau=\frac{A_{1}}{A_{s}}$

In this work we deposited peptide-amphiphile bilayers on mica substrates for AFM studies. The first layer was 1,2-disteraroyl-sn-glycero-3phosphoethanolamine (DSPE) (Avanti Polar Lipids, Inc) deposited on an upstroke, and the second layer was the respective fibronectin mimetic peptideamphiphile deposited on a downstroke. Peptide-amphiphiles were dissolved $\sim 1 \mathrm{mg} / \mathrm{mL}$ in chloroform solutions. DSPE was dissolved in chloroform at a concentration of $1.5 \mathrm{mg} / \mathrm{mL}$. A Langmuir-Blodgett (KSV Instruments, Helsinki, Finland) was used to deposit peptide-amphiphile bilayers on $7.5 \mathrm{~mm}$ mica disks. DSPE was deposited first oriented with hydrophobic tails facing away from the mica substrate. Next, a fibronectin mimetic amphiphile was deposited so that the hydrophilic heads were facing outwards. All depositions were performed with a surface pressure of $41 \mathrm{mN} / \mathrm{m}$, which is below the collapse pressure for all molecules, and at a speed of $1 \mathrm{~mm} / \mathrm{min}$. After peptideamphiphile deposition, the substrates were not exposed to air and were stored underwater for 1-2 days until AFM measurements were performed and were not exposed to air.

One difficulty encountered during deposition of the monolayer onto the DSPEcoated mica substrate was that the transfer ratio of PR_d was consistently low (Figure 2-9). The ideal transfer ratio is 1 . All the other peptide-amphiphiles had transfer ratios of $0.8-1.3$, indicating minimal disturbances during transfer; whereas, PR_d had a transfer ratio of 0.4. The low transfer ratio for PR_d might be due to the extensive hydrogen bonding between the serine residues, and instead of the monolayer coating the mica substrate, the PR_d molecules 
preferred to interact with each other and may allow the disk to slip through the monolayer without transferring the amphiphile.

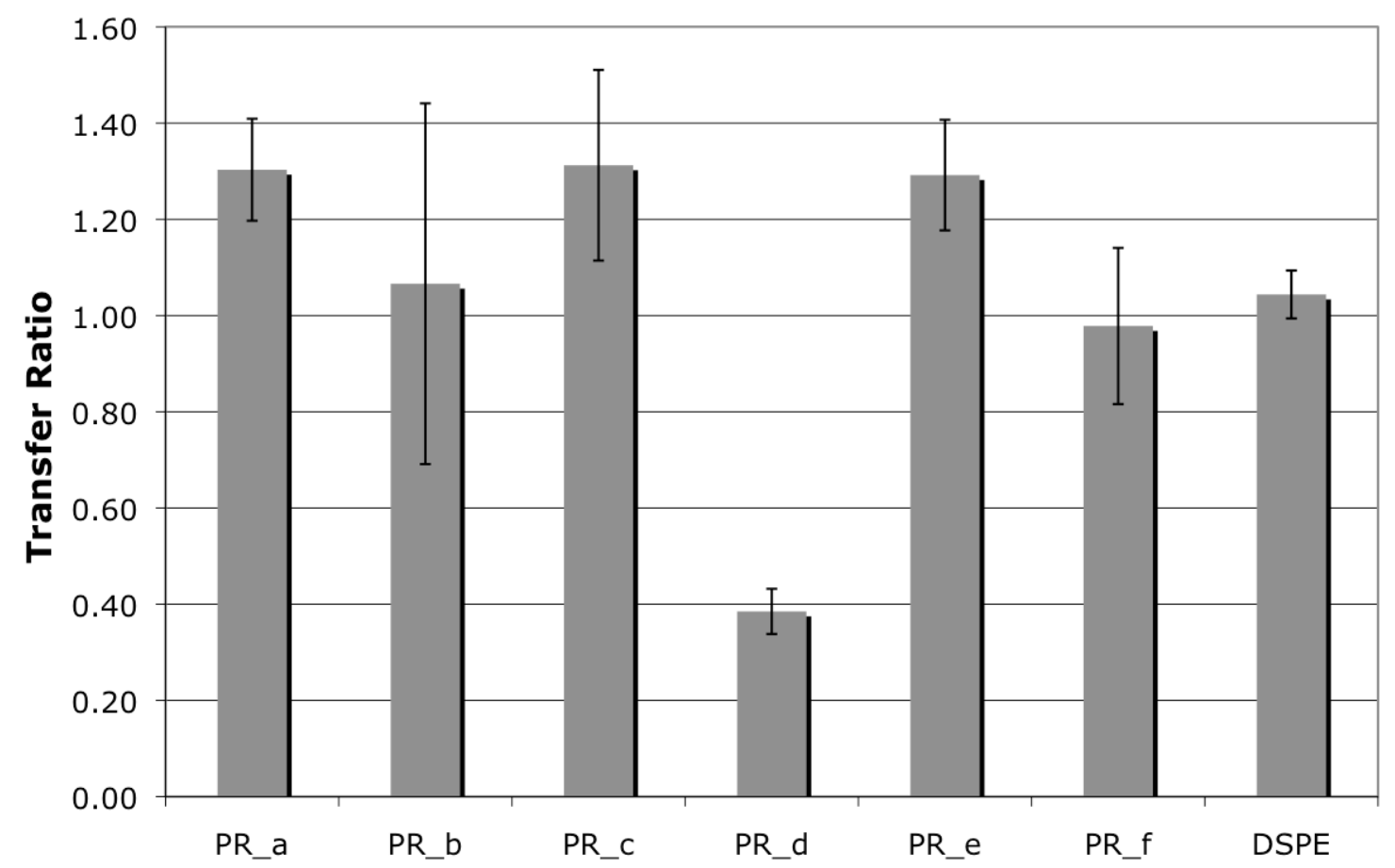

Figure 2-9: Average transfer ratios for the peptide-amphiphiles and DSPE lipid. PR_d has a particularly low transfer ratio possibly owing to the fact that it has ten serine residues, which form strong intermolecular hydrogen bonds and may prevent coating onto the mica substrate.

\subsection{Atomic Force Microscopy}

Atomic force microscopy (AFM) is a relatively old technique in the rapidly advancing field of nanotechnology. First developed in 1986 by G. Binning, the AFM is part of the large family of scanning probe microscopy (SPM). ${ }^{135,136}$ SPM is a type of microscopy that uses a physical probe to image a sample. AFM was developed to overcome limitations of scanning tunneling microscopy (STM), which requires a conductive sample. ${ }^{137}$ AFM uses a laser to measure the deflection of a tip on the end of a cantilever as the tip makes contact with a surface. The tip is raster-scanned over the surface to obtain an image. AFM 
has been used in a wide range of applications due to flexibility in sample choice. Samples can be examined in air or liquid allowing for samples to be imaged in their native environment. ${ }^{138,139}$ Originally, only hard samples could be scanned, but improvements in cantilever design and the development of tapping mode imaging has allowed very soft samples to be imaged such as living cells. ${ }^{136,140-142}$ AFM is capable of obtaining resolution on the nanometer scale allowing imaging of biomolecules such as DNA, ${ }^{143}$ proteins, ${ }^{144,}{ }^{145}$ and phospholipids. ${ }^{146,147}$ In addition to imaging, the physical contact between the probe and the sample allows for mechanical, chemical, and bioactivity properties to be analyzed. ${ }^{142}$

\subsubsection{Atomic Force Microscopy Fundamentals}

AFM utilizes a very small sharp tip on the end of a very flexible cantilever to probe the sample (Figure 2-10). The laser is reflected off the back of a cantilever onto a photodiode. ${ }^{148}$ The photodiode is divided into quadrants and is able to detect when the laser spot moves and thus can detect the deflection of the cantilever. In imaging mode, piezoelectric crystals move the sample in the $x-y$ plane under the tip in a raster fashion. A feedback control loop controls the sample-cantilever distance using piezoelectric crystals as the tip scans over the sample features. The AFM tip is deflected from the surface by a variety of forces. These forces include the long-range electrostatic and van der Waals forces and short-range repulsive forces. 


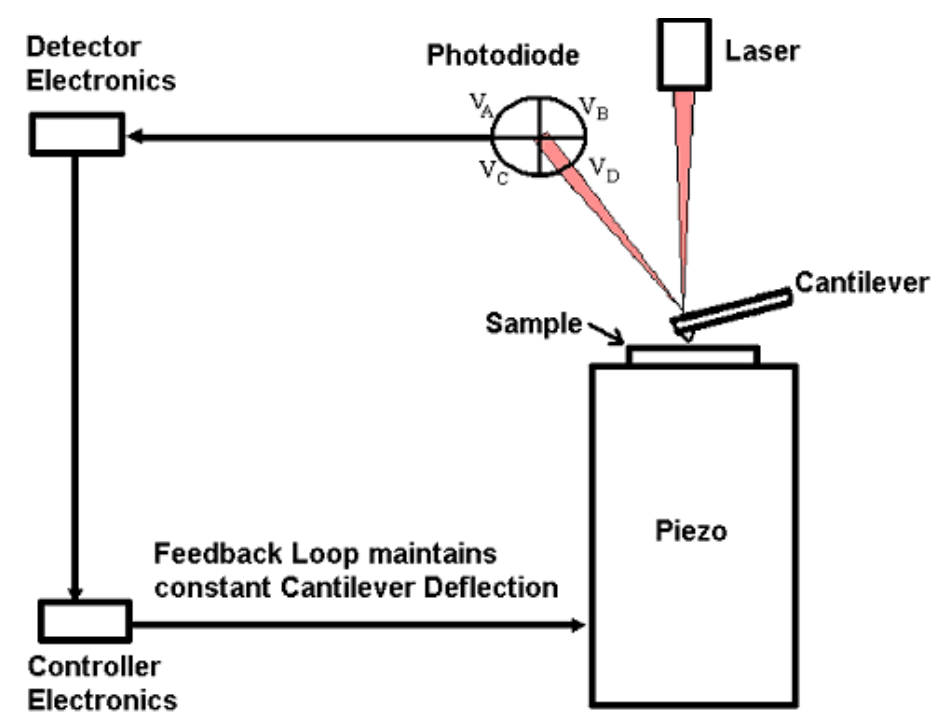

Figure 2-10: AFM schematic

AFM can be performed in many different operational modes. The most common modes are imaging and force. The imaging mode can be further divided into contact and tapping modes. In contact mode imaging, the tipsample interaction is maintained in the repulsive force regime, and the cantilever is dragged across the sample surface. The feedback control loop attempts to maintain a constant deflection, and the height of the sample is mapped by the vertical piezoelectric crystal position. The force exerted on the sample is directly proportional to the cantilever deflection by the cantilever spring constant. In order to image very soft samples using contact mode, a very soft cantilever with a low spring constant must be used. Contact imaging mode is capable of producing very high-resolution images especially when a slow scan rate is used. Friction between the tip and sample can result in torsion of the cantilever and give rise to a lateral difference in the photodiode signal. The lateral signal can indicate different chemical domains especially when using a chemically modified tip. 
The downside of contact mode is that lateral interactions give rise to dragging forces, which can damage delicate samples. As a result, biological samples are frequently imaged in tapping or intermittent-contact mode. In tapping mode, a small piezoelectric crystal is used to oscillate the cantilever near its resonance frequency. When the cantilever is brought near the surface, the amplitude of the vibration decreases due to the interactions of the tip with the surface. The tip can stay in the attractive regime for true non-contact imaging or it can cross into the repulsive regime and make some contact with the surface for intermittent-contact imaging. In tapping mode, the feedback control loop tries to maintain a constant amplitude. The phase shift between the driving oscillation and the cantilever oscillation is monitored to produce a phase image and can show variations in surface properties such as composition, adhesion, elasticity, and friction. ${ }^{149-151}$

In force mode, the AFM nanoindents the surface of a sample and obtains a force curve of distance moved by the piezoelectric crystal versus deflection of the cantilever as the tip approaches and retracts from the surface. This mode is very useful for determining the mechanical properties of the sample and adhesion forces between the tip and surface. As previously mentioned the deflection of the cantilever, $\Delta d$, is directly proportional to the indentation force, $F$, by Hooke's law, $F=k \Delta d$, where $\mathrm{k}$ is the cantilever spring constant. Hertzian mechanics can be applied to the approach curve to extract the sample elasticity. ${ }^{152-154}$ The cantilever tip can be functionalized with adhesion molecules and the binding between a ligand and receptor seen in the retract curve can be characterized by the force required to break the bond. ${ }^{99,155,156}$ 


\subsubsection{AFM Imaging of Fibronectin Mimetic Peptide-Amphiphile Langmuir Blodgett Supported Bilayers}

LB surfaces were prepared on mica substrates as described in Section 2.5.3. The prepared surfaces were stored in Milli- $Q$ water for up to two days before AFM imaging. Tapping mode images were obtained using a Digital Instruments (Veeco) Nanoscope III AFM fitted with a fluid cell and a J-type scanner. The surfaces were positioned in the fluid cell without exposing the imaging surface to the air. AFM images of the surfaces were obtained in tapping mode using the NPS-series short fat cantilever with silicon nitride tip. (Veeco Probes, Camarillo, CA). The cantilever was vibrated at $69.7 \mathrm{kHz}$, which was $10 \%$ below the peak resonant frequency. The fluid cell was filled with a $1 \mathrm{mM} \mathrm{MnCl}_{2}$ solution to be consistent with receptor-ligand binding experiments performed in parallel by other group members. Images were recorded at scan sizes of $10 \mathrm{x}$ $10 \mu \mathrm{m}$ and $30 \times 30 \mu \mathrm{m}$. The scan rate was $1.5 \mathrm{~Hz}$ for PR_a and $2 \mathrm{~Hz}$ for the rest of the peptide-amphiphiles. First or second order flattening was applied to all images to remove image artifacts such as scan lines and surface tilt. 

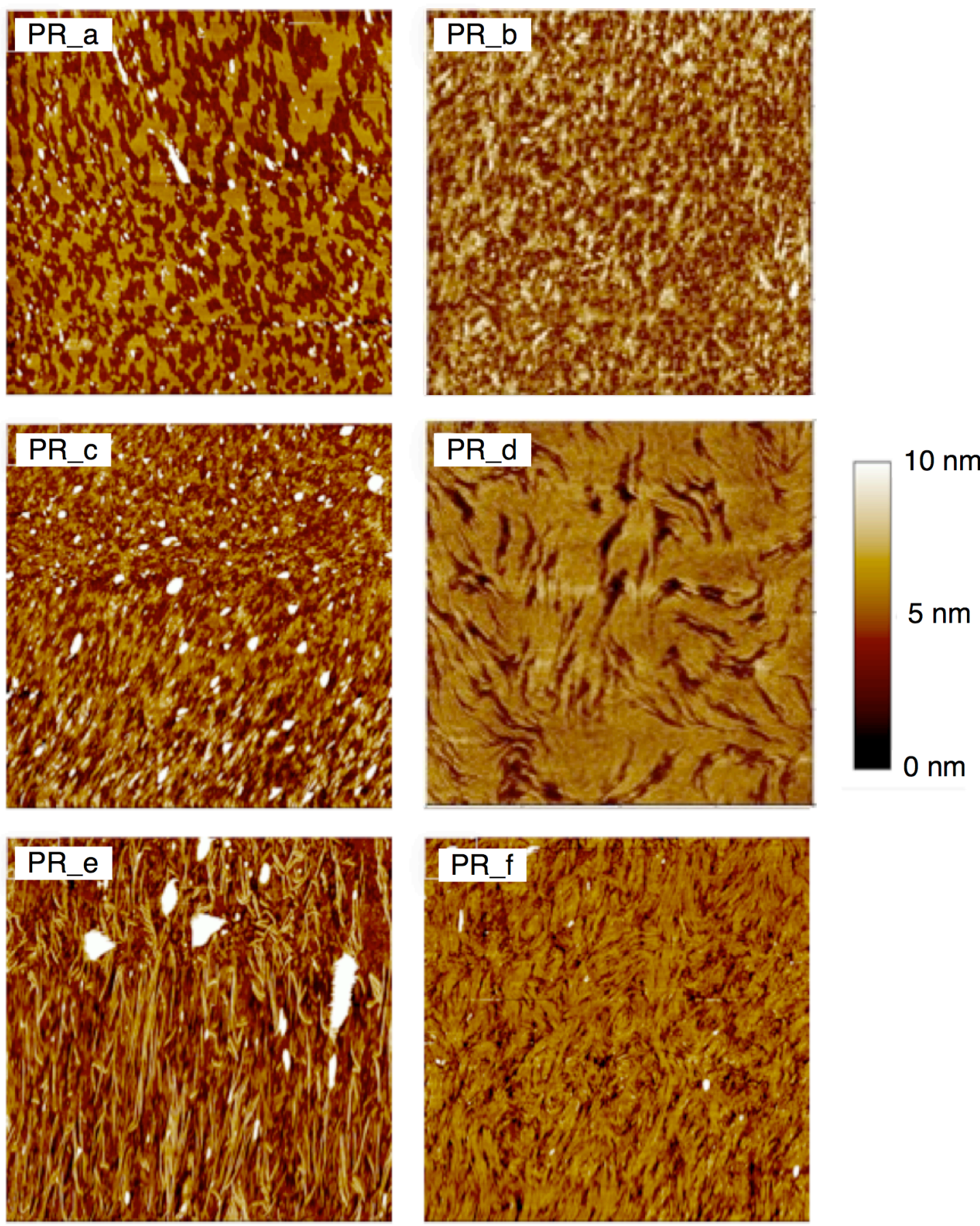

Figure 2-11: AFM tapping mode height images of the peptide-amphiphile bilayers 
AFM height images of the peptide-amphiphile monolayers are shown in Figure 2-11. These images are similar to previous images of the PR_a. ${ }^{119}$ The image of PR_c shows some stretching effects due to the fluid cell O-ring on the cantilever. When imaging using a fluid cell, occasionally the O-ring, which keeps a watertight seal, will prevent the piezoelectric crystal from moving the surface precisely resulting in some stretching artifact in the image. PR_a, PR_b, PR_c, PR_d, and PR_f show evidence of shallow holes or divots in the membrane. Cross-sectional analysis shows that these are approximately $3 \mathrm{~nm}$ deep (Table 2-3).

Table 2-3: Depth of divot features in the AFM images of the fibronectin mimetic peptide-amphiphile films. The theoretical peptide lengths are calculated assuming that the peptide assumes a random secondary structure and that the length of each amino acid is $0.37 \mathrm{~nm} .{ }^{98,} 118{ }^{*}$ Circular dichroism spectroscopy of PR_d indicates that its secondary structure contains some a-helix and $\beta$-sheet folding, and the theoretical length of PR_d is dependent upon the relative amounts of each structure. ${ }^{157}$ The reported error is the standard deviation.

\begin{tabular}{|c|c|c|}
\hline Name & Depth of Divots $(\mathbf{n m})$ & $\begin{array}{c}\text { Theoretical Peptide } \\
\text { Headgroup Length } \mathbf{( n m})\end{array}$ \\
\hline PR_a & $2.83 \pm 0.39$ & 6.66 \\
\hline PR_b & $3.10 \pm 0.37$ & 8.51 \\
\hline PR_c & $3.13 \pm 0.36$ & 8.51 \\
\hline PR_d & $3.23 \pm 0.57$ & $3.45-8.05^{\star}$ \\
& $7.09 \pm 1.13$ & 7.40 \\
\hline PR_e & & 9.62 \\
\hline PR_f & $3.33 \pm 0.67$ & \\
\hline
\end{tabular}

Circular dichroism (CD) spectroscopy confirms that PR_a, PR_b, PR_c, PR_e, and $P R \_f$ all exhibit a random secondary structure, ${ }^{157}$ so the theoretical peptide headgroup length can be calculated assuming $0.37 \mathrm{~nm}$ per amino acid. ${ }^{98,} 118$ 
The hydrophobic tail is approximately $3.0 \mathrm{~nm}$ long fully extended, calculated assuming a C-C bond length of $1.54 \AA$ and bond angle of $109^{\circ}$. The depth of the divots seen in the surface of $P R \_a, P R \_b, P R \_c$, and $P R \_f$ is not as deep as the length of the peptide-amphiphile. This suggests that the divots are more likely to be peptide-amphiphiles with the peptide headgroups bent or lying down as opposed to missing sections of peptide-amphiphile. The bend could be a result of the proline residue in the PHSRN domain, which has a constrained structure. If the holes were defects in the Langmuir-Blodgett film where no peptide-amphiphile was transferred, the depth of the hole would be closer to $10 \mathrm{~nm}$ deep. PR_d has evidence of two depths in the divots. The first depth of $3.23 \pm 0.57 \mathrm{~nm}$ is similar to depths seen in PR_a, PR_b, PR_c, and PR_f. The second depth of $7.09 \pm 1.13 \mathrm{~nm}$ is closer to the theoretical length of the peptideamphiphile and may be evidence of defects in the bilayers. The low transfer ratio of $P R \_d$ supports the hypothesis that $P R \_d$ may have areas of missing peptide-amphiphile. PR_e seems to have some fibril contamination on the surface. Underneath the fibrils, cross-sectional analysis shows some of the same approximately $3 \mathrm{~nm}$ deep holes as the other peptide-amphiphile surfaces, but the image is not clear enough to draw distinct conclusions. PR_d and PR_f show interesting swirl patterns. The cause of the patterns on the PR_d and PR_f surfaces is unknown.

\subsection{Cell and Integrin Adhesion to PR_a - PR_f Langmuir- Blodgett Supported Surfaces}

In parallel with the above work, Jennifer Craig plated HUVEC cells on the fibronectin mimetic peptide-amphiphile surfaces and examined the cell adhesion at 1,4 , and 12 hours. ${ }^{157}$ Two experiments were performed, one comparing the effect of the hydrophilicity/hydrophobicity of the linker region and another comparing the effect of the length of the spacer. PR_b outperformed 
the other surfaces, especially after 12 hours, indicating that hydrophilicity/hydrophobicity of the $(S G)_{5}$ linker is more favorable for HUVEC adhesion than $G_{10}$ and $S_{10} .{ }^{157}$ The effect of the spacer was less pronounced than the effect of the linker. PR_e performed nearly as well as PR_b, but PR_f did not adhere cells as well as PR_b and PR_e. ${ }^{157}$ The KSS spacer of PR_b seems to be the best length for the spacer for HUVECs adhering to a supported bilayer.

Anastasia Mardilovich examined the binding strength between the fibronectin mimetic peptide-amphiphile Langmuir-Blodgett films and $\alpha_{5} \beta_{1}$ integrin using an AFM in force mode. ${ }^{157}$ PR_b showed a much greater adhesion to the $a_{5} \beta_{1}$ integrin-functionalized cantilever tips than any of the other fibronectin mimetic peptide-amphiphiles. ${ }^{157}$ This indicates that PR_b may have a higher degree of specificity for the $a_{5} \beta_{1}$ integrin and validates the hypothesis that using an (SG) linker can increase cell adhesion to the fibronectin mimetic peptide. ${ }^{157}$

\subsection{Conclusion}

One of the main challenges in biomaterial and tissue engineering design is encouraging cell adhesion. The most commonly used method is the incorporation of biomimetic peptide sequences that interact with cell surface receptors. This chapter described the design of fibronectin mimetic peptideamphiphiles that contained the primary binding domain, RGD, and the synergybinding domain, PHSRN, found in fibronectin. Cells recognize PHSRN and RGD using the cell surface receptor called $a_{5} \beta_{1}$ integrin. Previously, a fibronectin peptide-amphiphile, PR_b was designed with a linker between the two binding domains that mimicked both the separation length found in the native fibronectin and the hydrophilicity/hydrophobicity of the amino acids found between the two domains. This work compared the $(S G)_{5}$ linker of PR_b with sequences that had all-glycine or all-serine linkers. PR_b also contained a 
spacer to extend the PHSRN domain from the hydrophobic tails, and the effect of the spacer length was compared with sequences that contained no spacer and a longer spacer than PR_b.

The peptides were synthesized using standard Fmoc solid-phase peptide synthesis, coupled to a dialkyl tail, and purified with HPLC. The LangmuirBlodgett technique was used to transfer the peptide-amphiphiles to a mica substrate for AFM experiments. Langmuir isotherms of the peptide-amphiphiles revealed that peptide-amphiphiles with a large number of serine residues had a high degree of intermolecular interaction. This led to a poor transfer of PR_d to the mica substrate. AFM images showed that the mica surfaces were well covered with the exception of PR_d. The surfaces all exhibited "divots" that are likely areas were the headgroups were bent or partially laying down. These areas may provide $a_{5} \beta_{1}$ integrins better access to the PHSRN domain. Parallel experiments conducted by other members of the Kokkoli lab group showed that PR_b was the superior sequence for $a_{5} \beta_{1}$ integrin binding as measured by functionalized AFM tips and for HUVEC cell binding.

$P R \_b$ has shown to be an excellent fibronectin mimetic sequence in $2 D$ experiments. However, most of cellular interactions in the body require a 3D environment to properly mimic native tissues. The next chapters build upon the experience gained from the design of $\mathrm{PR} \_\mathrm{b}$ and incorporate the fibronectin mimetic sequence into 3D scaffolds for tissue engineering applications. 


\section{Chapter 3 Peptide-Amphiphile Nanofibers}

\subsection{Chapter Overview}

The next goal of this research project was to use the fibronectin mimetic peptide sequence in a scaffold for tissue engineering applications. Peptide-amphiphiles with single tails have been shown to self-assemble into nanofibers and even form gels, which may be used, for example, as a scaffold for wound repair. This chapter examines peptide-amphiphile nanofiber structures as previously studied, then outlines the design of a modified fibronectin mimetic peptideamphiphile designed to form nanofibers in solution.

\subsection{Nanofibers}

Peptide-amphiphiles have been shown to assemble into a wide variety of shapes. Cylindrical micelles, also called nanofibers, have been shown to form gels under certain conditions, which may be used as scaffolds for tissue engineering applications. One of the first examples of peptide-amphiphile nanofibers was described by Hartgerink et. al. in 2001, and these nanofibers were designed to encourage oriented hydroxyapatite deposition for bone tissue engineering. ${ }^{81}$ The researchers coupled a palmitic acid $\left(C_{16}\right)$ to a hydrophilic peptide headgroup, and they discovered that by altering the $\mathrm{pH}$, the peptideamphiphile could self-assemble into cylindrical micelles and form a gel. As previously mentioned, one of the advantages of peptide-amphiphiles is the biomimetic design flexibility granted by the peptide headgroup. In addition to encouraging hydroxyapatite deposition, ${ }^{81,158}$ other peptide sequences have been incorporated into peptide-amphiphiles to encourage the binding of heparin to promote angiogenesis, ${ }^{159-161}$ and some peptide-amphiphile nanofibers contain cell adhesion domains to encourage cell growth and 
proliferation. ${ }^{101-103,162}$ Cells can be encapsulated in peptide-amphiphile gels, and TEM images show evidence that cells internalize the peptideamphiphiles, ${ }^{102}$ which may be a method of passive degradation. Active degradation of peptide-amphiphile gels can be achieved by incorporating a proteolytic sequence into the peptide headgroup. ${ }^{163}$ Dental stem cells encapsulated in peptide-amphiphile gels containing a matrix metallopeptidase 2 (MMP2) peptide sequence were able to form either hard or soft dental tissue depending on the stem cell line introduced. ${ }^{164}$ A peptide-amphiphile bearing the laminin mimetic peptide, IKVAV, was able to encourage the selective differentiation of neural progenitor cells into neurons, as opposed to astrocytes, and showed neurite growth after only one day in culture. ${ }^{162}$ This peptideamphiphile was injected in a mouse with a spinal cord injury. The peptideamphiphile gel reduced apoptotic cells at the injury site and reduced glial scar formation. This allowed for a greater axonal growth even after the degradation of the peptide-amphiphile. ${ }^{165}$ In addition to gel formation, peptide-amphiphile nanofibers have been used as coatings for carbon nanotubes ${ }^{100}$ and polyglycolic acid (PGA) scaffolds ${ }^{166}$, and they are able to be patterned on surfaces using techniques such as dip-pen patterning ${ }^{167}$ and sonicationassisted solution embossing. ${ }^{168}$

\subsubsection{Mechanisms of Self-Assembly}

In order to induce self-assembly of peptide-amphiphiles into nanofibers, researchers have employed a variety of methods to counteract the charge of the peptide headgroup. The first studies altered the $\mathrm{pH}$ of the peptideamphiphile solution to near the peptide-amphiphile's isoelectric point. ${ }^{81}$ At the isoelectric point the researchers noticed the formation of nanofibers. When the $\mathrm{pH}$ of the solution was changed so that the peptide-amphiphile became charged again, the nanofibers disassembled. Some nanofibers were designed 
containing cysteine residues, which could be cross-linked stabilizing the nanofibers with disulfide cross-links, and after cross-linking, the nanofibers were stable to $\mathrm{pH}$ changes. ${ }^{101}$ Most biological systems are sensitive to drastic changes in $\mathrm{pH}$, so another method was devised to counteract the charge by adding counterions to the peptide-amphiphile solution. ${ }^{101,102,169}$ Most of the studies focused on adding divalent cations to negatively charged peptideamphiphiles. The mechanical properties of the gel were able to be tuned by changing the cation concentration ${ }^{169}$ or by changing the type of cation used. ${ }^{102,169}$ Assembly by divalent cations is particularly attractive for biological systems because cell media and interstitial fluid both contain a sufficient cation concentration to encourage self-assembly. Another published method to counteract the peptide-amphiphile charge was to mix a negatively-charged peptide-amphiphile with a positively-charged peptide-amphiphile. The oppositely-charged peptide-amphiphiles counteracted each other's repulsive charge and coassembled into nanofibers. ${ }^{103,170}$ A few studies have described including a photolabile side group to the peptide-amphiphile, which prevents self-assembly until after photoinitiation. ${ }^{171,172}$ This offers exact control over the timing of the self-assembly of the peptide-amphiphiles.

\subsubsection{Nanofiber Structure}

Surfactant self-assembly is a widely studied area of colloids. The selfassembled shape is dependent upon the molecular shape of the surfactant. ${ }^{77}$ In general, single-tailed surfactants form micellar structures and double-tailed surfactants form bilayers structures. The theoretical shape can be determined by calculating the critical packing number. Truncated cones, which have a single hydrocarbon tail and a small headgroup cross section have a critical packing number between $1 / 3$ and 1/2 should self-assemble into cylindrical micelles. ${ }^{77}$ Peptide-amphiphiles have a very large headgroup, yet they 
preferentially form nanofibers after self-assembly seemingly contradictory to surfactant theory. Also, some have argued that all surfactants with a conical shape, even truncated ones, should form spherical micelles in the absence of attractive charges in the headgroup. ${ }^{173}$ Molecular dynamic simulations suggest that electrostatic interactions found in the headgroups of peptide-amphiphiles may help the formation of cylindrical micelles. ${ }^{174}$ Peptide headgroups usually contain both positively- and negatively- charged groups and may exhibit attractive electrostatic interactions; whereas, most simple surfactants are not zwitterionic and are only capable of repulsive electrostatic interactions. In addition to simple cylindrical micelle nanofibers, peptide-amphiphiles have also been shown to form other nanofiber shapes such as twisted ribbons ${ }^{78}$ and helices. ${ }^{175,176}$

The basic structure of a peptide-amphiphile nanofiber is that of a cylindrical micelle (Figure 3-1). The hydrophobic tails are oriented toward the interior of the fiber, and the hydrophilic peptide headgroup forms the outer shell of the nanofiber, which was verified in several transmission electron microscopy (TEM) studies showing cross-sections of nanofibers, which show that uranyl acetate only stains the exterior portion of the nanofiber and not the interior. ${ }^{81,} 102$ Uranyl acetate binds to acid groups found in the peptide headgroup. Fluorescence experiments looking at the fluorescent signature of tryptophan showed that the peptide headgroup is highly hydrated along the entire length of the peptide even very close to the core. ${ }^{177}$ Even though the shell is highly hydrated, diffusion in the innermost parts of the shell is much slower than the outermost layer. ${ }^{177}$ Similar experiments using pyrene showed that significantly less water is found inside the hydrophobic core of the nanofiber. ${ }^{177}$ 


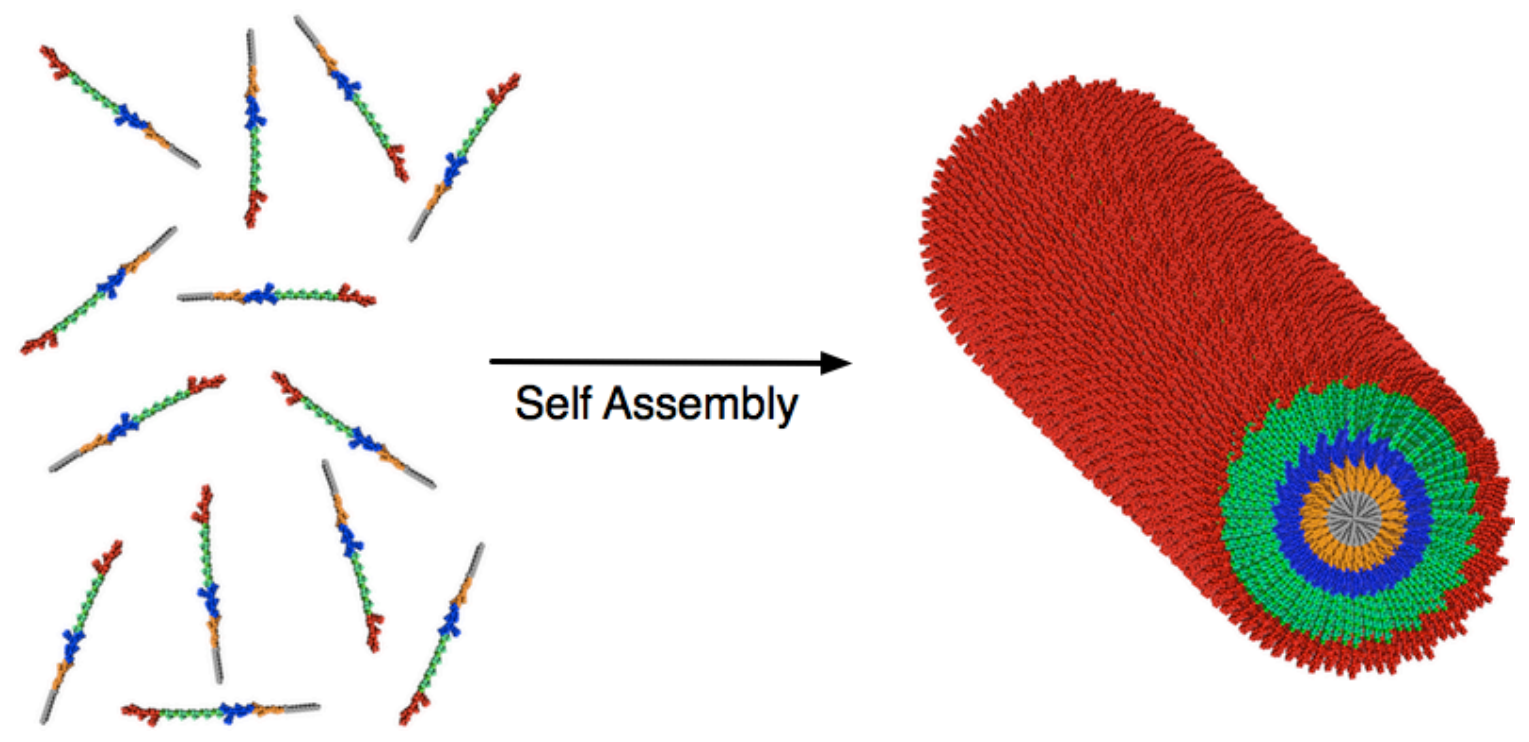

Figure 3-1: Schematic of self-assembly of peptide-amphiphile molecules into a nanofiber. The hydrocarbon tails form the interior of the nanofiber and the peptide headgroup forms the exterior shell.

The diameters of the nanofibers have been measured by TEM and have been reported to be between 7-11 $\mathrm{nm}$ for hydrocarbon tails sixteen carbons long and peptide headgroups 9-13 amino acids long. ${ }^{103,171,178-180}$ Assuming, a fullyextended tail conformation (1.54 $\AA \mathrm{C}-\mathrm{C}$ bond lengths and $109^{\circ}$ bond angle) and an unordered headgroup structure (3.7 ̊/amino acid), ${ }^{98,}{ }^{118}$ the theoretical lengths of these peptide-amphiphiles is $5.3-6.8 \mathrm{~nm}$ leading to theoretical nanofiber diameters of approximately $10-14 \mathrm{~nm}$. A hydrocarbon tail with a sixteen-carbon $\left(\mathrm{C}_{16}\right)$ backbone is the most commonly used length in nanofiberforming peptide-amphiphiles. One study showed that hydrocarbon tails shorter than $\mathrm{C}_{12}$ do not allow for the proper secondary structure to form in the peptide headgroup; therefore, the peptide-amphiphiles do not form stable nanofibers. ${ }^{78}$ The minimum length of the hydrocarbon tail is dependent upon the peptide headgroup composition, and another study shows that tail lengths shorter than $\mathrm{C}_{10}$ were unable to form gels. ${ }^{101}$ 


\subsubsection{Peptide Headgroup Secondary Structure}

Proteins fold into a wide variety of spatial conformations driven by electrostatic, $\mathrm{H}$-bond, and hydrophobic interactions between their constitutive amino acids. Short peptides are capable of forming a variety of secondary structures such as $a$-helices and $\beta$-sheets, and the secondary structure of the peptides can be determined through spectroscopy techniques. Fourier transform infrared spectroscopy (FT-IR) studies of coassemblies of peptide-amphiphiles forming nanofibers indicated strong hydrogen bonding in the $\mathrm{N}-\mathrm{H}$ stretch and showed significant signs of parallel $\beta$-sheet formation. ${ }^{103}$ This evidence was corroborated in many circular dichroism (CD) spectroscopy experiments, which showed that many peptide-amphiphiles exhibit a $\beta$-sheet secondary structure upon self-assembly into nanofibers. ${ }^{78,170,178-180}$

To determine the effect of the secondary structure on the peptide-amphiphile self-assembly, Paramonov et. al. synthesized a series of peptide-amphiphiles containing $\mathrm{N}$-methylglycines (sarcosine) in place of glycine residues. ${ }^{181} \mathrm{~N}$ methylglycine contains a methyl group attached to the nitrogen instead of a hydrogen atom, which effectively prevents hydrogen bonding with neighboring peptide headgroups by eliminating the hydrogen donor. They found that if a Nmethylglycine was located in the four amino acids closest to the hydrocarbon tail, the peptide-amphiphile was unable to form a gel. If $\mathrm{N}$-methylglycine replaced the $5^{\text {th }}$ amino acid, the peptide-amphiphile was able to only form a weak gel. If only one substitution of $\mathrm{N}$-methylglycine for a glycine was made in the first four amino acids, the peptide-amphiphiles still retained the capability to form fibers, but they were still unable to gel. With more than one substitution, the peptide-amphiphile was unable to form fibers. They also synthesized a series of peptide-amphiphiles containing an alanine residue replacing a glycine and showed by difference CD spectroscopy that an alanine residue located 
within the first four amino acids is in a $\beta$-sheet conformation, and after the first four amino acids the alanine participates in a polyproline type II structure. ${ }^{181}$ This study provided strong evidence that the $\beta$-sheet structure within the first 4-5 amino acids is crucial for nanofiber formation and disrupting the hydrogen bonding in the $\beta$-sheet will prevent nanofiber formation and gelation. Further evidence that the $\beta$-sheet stabilizes the nanofibers is that peptide-amphiphiles that showed $\beta$-sheet structure prior to inducing self-assembly gelled more quickly than peptide-amphiphiles showing a unordered structure prior to assembly. ${ }^{182}$ Also, peptide-amphiphiles that have more $\beta$-sheet characteristics have more highly ordered hydrophobic cores, which imparts greater stability to the nanofiber. ${ }^{183}$

Polarization modulation-infrared reflection-adsorption spectroscopy ${ }^{183}$ and infrared (IR) spectroscopy measurements performed at a grazing angle ${ }^{181}$ showed that the $\beta$-sheet forms along the longitudinal axis of the nanofiber. Some measurements have indicated that the $\beta$-sheet may have a slight twist along the longitudinal axis ${ }^{181,183}$ as seen in amyloid fibers ${ }^{184}$ and $\beta$-sheetforming peptide fibrils. ${ }^{185}$ Monte Carlo simulations of peptide-amphiphiles showed $\beta$-sheet formation along the longitudinal axis, and the simulations suggested that the $\beta$-sheet secondary structure leads to the cylindrical shape of the nanofibers. ${ }^{186}$

In addition to the $\beta$-sheet secondary structure, some peptide-amphiphiles have shown a little $a$-helical content in CD spectra. ${ }^{179}, 180$ Peptide-amphiphiles containing a greater glycine content tended to show more a-helix. ${ }^{180} \mathrm{CD}$ and IR spectra of other peptide-amphiphiles have shown evidence of polyproline type II structures in addition to $\beta$-sheet secondary structure. ${ }^{181}$ 


\subsection{PR_g Design}

Our goal was to make a fibronectin mimetic peptide-amphiphile capable of forming gels for tissue engineering by expanding upon the PR_b project and targeting $\alpha_{5} \beta_{1}$ integrin with PHSRN and RGD in a three-dimensional scaffold. One peptide-amphiphile nanofiber study designed a fibronectin mimetic peptideamphiphile with a single tail containing both PHSRN and RGD. The two domains formed two arms of a branched peptide-amphiphile, but the sequence did not adhere cells any better than a simple RGD sequence. ${ }^{166}$ We designed a peptide-amphiphile incorporating the lessons learned studying PR_a - PR_f with a single hydrocarbon tail. While PR_b's peptide headgroup has been shown to be very effective in both drug delivery and cell culture experiments, the sequence is not designed for nanofiber formation, and we decided to approach the design from the bottom up. The new peptide-amphiphile was designed with the peptide sequence GGGSSPHSRN(SG) ${ }_{5}$ RGDSP and a single $\mathrm{C}_{16}$ hydrocarbon tail and was called PR_g (Figure 3-2). The design considerations are discussed below.

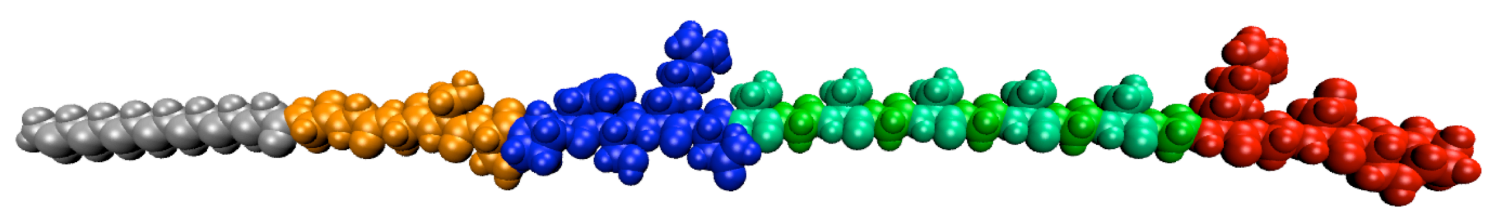

\section{$\mathrm{C}_{16}$ Tail GGgSSPHSRNGSGSGSGSRGDSP}

Figure 3-2: PR_g fibronectin mimetic peptide-amphiphile structure. The amphiphile has a palmitoyl tail coupled to a GGGSS spacer, PHSRN synergy-binding domain, (SG) $)_{5}$ linker, and RGDSP primary binding domain. 


\subsubsection{PR_g Binding Domains, Linker Region, and Tail}

As discussed in Chapter 2, RGD has been identified as the primary binding domain for $a_{5} \beta_{1}$ integrin to fibronectin, and PHSRN is the synergy-binding domain. Studies with fibronectin mimetic peptides have suggested that peptide mimetics that contain PHSRN on the $\mathrm{N}$-terminal side of RGD perform better than if the RGD domain is located before the PHSRN. ${ }^{115}$ Fibronectin mimetic peptides also perform better when the two domains are coupled by a flexible linker that accurately mimics the $30-40 \AA$ distance ${ }^{109}$ between the two binding domains. ${ }^{114,115,119}$ PR_g was designed to have the same flexible (SG) $)_{5}$ linker as PR_b to mimic the distance between the PHSRN and RGD domains. Comparisons between PR_b, PR_c, and PR_d showed that the alternating hydrophobic/hydrophilic amino acid of the $(S G)_{5}$ linker favored HUVEC attachment. ${ }^{157}$ As with all the previously tested peptide-amphiphiles, PR_g contains a C-terminal amide to prevent electrostatic repulsions between the negatively charged cell surface and the C-terminus peptide-amphiphile. To encourage cylindrical micelle formation as opposed to bilayers structures, a single hexadecanoic acid hydrocarbon tail was coupled to the $\mathrm{N}$-terminus of the peptide headgroup. Hexadecanoic acid was chosen because it is the most commonly used in the literature and is long enough to promote stable nanofiber formation. ${ }^{78,101}$

\subsubsection{PR_g Spacer Design}

The only difference between the PR_g and PR_b headgroups is the spacer between the hydrocarbon tail and the PHSRN domain. In order to form stable nanofibers, the peptide headgroup must allow $\beta$-sheet formation in the first 4-5 amino acids. Each amino acid has a preference for a specific secondary structure, and this conformational preference is called its propensity. The first propensity scales were developed by examining the secondary structures of 
proteins and peptides and also by analyzing the appearance frequency the constitutive amino acids. ${ }^{187,} 188$ Amino acids with bulky side chains such as valine, isoleucine, threonine, phenylalanine, tyrosine, and tryptophan were found to occur in $\beta$-sheet secondary structures. ${ }^{187}$ The hydrophobic interactions between the side groups are thought to stabilize the $\beta$-sheet formation. ${ }^{188-191}$ Alanine, leucine, methionine, histidine, lysine, arginine, glutamine, and glutamic acid tend to be found in a-helical structures. ${ }^{187,192}$ Amino acids with short, polar side chains such as serine, aspartic acid, and asparagine along with the "special" amino acids proline and glycine tend to form turns and bends. ${ }^{187,} 192$ Proline is a spatially constrained amino acid due to the nature of its side chain and tends to initiate the turns. ${ }^{192}$ A very low propensity indicates that an amino acid is not suited to forming a certain secondary structure. Aspartic acid, asparagine, glutamic acid, glutamine, lysine, and proline have been identified as amino acids that will prevent the formation of $\beta$-sheets in proteins. ${ }^{187,} 192$ Serine also shows a low occurrence in $\beta$-sheets in native proteins, but substitution studies have shown that serine favors $\beta$-sheet formation. ${ }^{188}$

PR_b contains a KSS spacer followed by the PHSRN domain. This sequence has two amino acids in the first four amino acids (lysine and proline), which prevent $\beta$-sheet formation and might prevent a single-tailed PR_b peptideamphiphile from forming nanofibers. The lysine residue could be removed from the spacer, but the proline residue is part of the synergy-binding domain and cannot be eliminated. So more space was needed between the hydrophobic tail and the PHSRN. Thus, a new spacer was designed to be 5 amino acids long pushing the proline to the sixth residue. The amino acids that form $\beta$ sheets in native proteins are highly hydrophobic amino acids and are not optimal for nanofiber formation though because the peptide headgroup needs to maintain an overall hydrophilic character, and the bulky side chains may prevent cylindrical micelle self-assembly. Also, increasing the overall 
hydrophobicity may decrease the peptide-amphiphile's water solubility. While $\beta$-sheet formation is thought to be stabilized by the hydrophobic side groups, the hydrophobic tail of the peptide-amphiphile may provide enough stabilization to allow the other amino acids to form $\beta$-sheets. Other studies have designed nanofiber-forming peptide-amphiphiles containing the first four amino acids GGGG, ${ }^{181}$ AAAA,${ }^{101}$ CCCC,${ }^{81}$ GTAG, ${ }^{163}$ LLLK, ${ }^{179}$ and VVVV. ${ }^{170}$ Since lysine has been identified as a $\beta$-sheet breaker, ${ }^{187}$ the residue was removed from the spacer and replaced by three glycine residues. The decision to remove the lysine instead of adding a spacer to push the lysine further from the hydrophobic tail was also motivated by the increasing length of the peptide headgroup. PR_g is 25 amino acids long, which is five amino acids longer than the longest nanofiber-forming peptide-amphiphiles reported in the literature. ${ }^{170}$ Glycine and serine are very flexible residues that are indifferent to the formation of $\beta$-sheets and are expected to allow $\beta$-sheet formation in nanofibers.

\subsubsection{PR_g Charge}

PR_g has a theoretical charge of +1 at neutral pH (Figure 3-3). PR_b has a theoretical charge of +2 at neutral $\mathrm{pH}$. Most nanofiber-forming peptideamphiphiles are designed with a negative charge, but positively-charged peptide-amphiphiles have been shown to self-assemble into nanofibers with the addition of $\mathrm{KCl}$ solution ${ }^{102}$ or co-assembly with negatively-charged peptideamphiphiles. Studies have indicated that a lower charge may lead to higher nanofiber stability. ${ }^{170}$ 


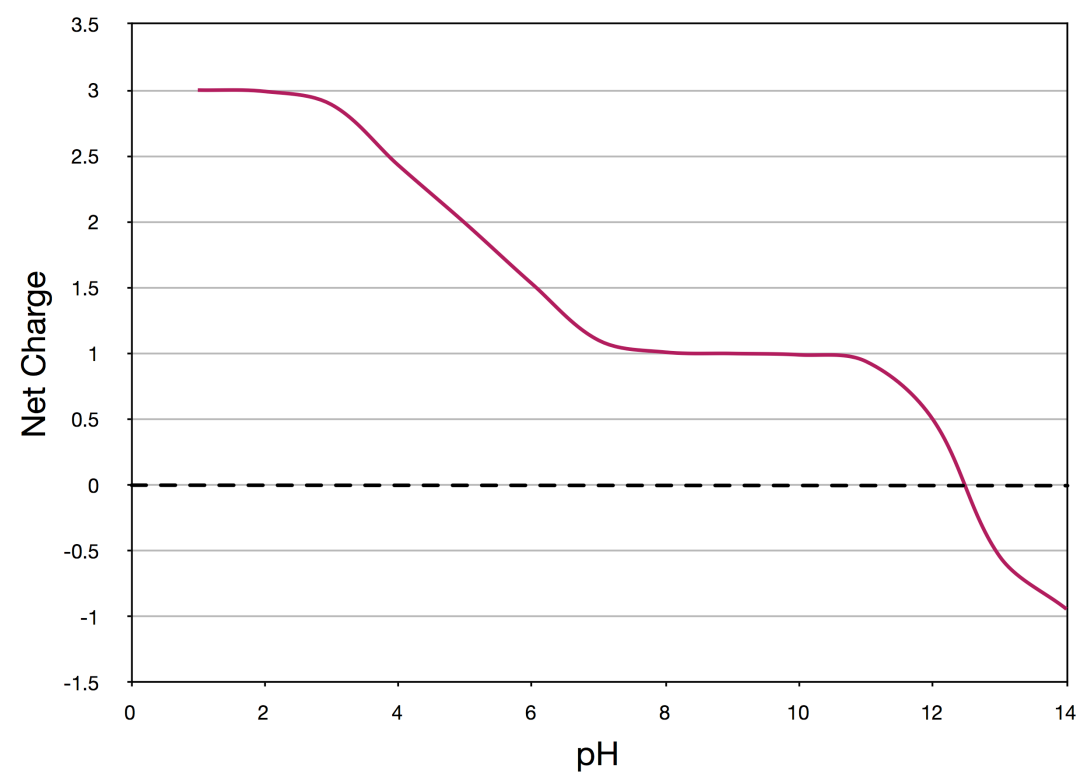

Figure 3-3: Theoretical charge of PR_g at various $\mathrm{pH}$. Net charge is calculated based on the pKa values for the constitutive amino acids. ${ }^{193}$ 


\section{Chapter 4 Synthesis \& Characterization of PR_g}

\subsection{Chapter Overview}

This chapter describes the characterization of the designed PR_g peptideamphiphile. The peptide-amphiphile was synthesized via solid phase peptide chemistry and purified with HPLC. The peptide-amphiphile self-assembled structure was analyzed with cryo-transmission electron microscopy and smallangle neutron scattering and was found to form nanofibers in solution. Circular dichroism spectroscopy was employed to examine the secondary structure of the peptide and peptide-amphiphile. The peptide-amphiphile appeared to form a self-supporting gel at higher concentrations, and the gel mechanics were investigated with oscillating plate rheometry.

\subsection{PR_g Synthesis}

An Fmoc-protected PR_g peptide was synthesized by the University of Minnesota's Oligonucleotide and Peptide Synthesis Facility using standard Fmoc solid phase peptide chemistry. Peptides were synthesized on a PAL resin, which results in a C-terminal amide after cleavage. All synthesis chemicals were obtained from Sigma-Aldrich with the exception of HBTU, which was obtained from EMD Biosciences and DIPEA, which was obtained from Fischer Scientific. Water was obtained from a Milli-Q water system and was purified to a resistivity of $18.2 \mathrm{M} \Omega \cdot \mathrm{cm}$.

As stated before, the structure of the hydrocarbon tail has an impact on the selfassembled structure of the peptide-amphiphile. In order to induce the PR_g peptide-amphiphile to form cylindrical micelles, a single $\mathrm{C}_{16}$ hydrocarbon tail was attached to the peptide headgroup. Hexadecanoic acid contains a 
carboxylic acid end group that allows the same coupling chemistry to be used as peptide synthesis resulting in the formation of an amide bond between the peptide and tail (Figure 4-1). First, the Fmoc protecting group was removed in a $20 \%$ solution of piperidine in DMF. The presence of a free $\mathrm{N}$-terminus was verified by a Kaiser test. The tail was coupled to the peptide on the beads in a solution of three times excess hexadecanoic acid, three times excess HBTU, and six times excess DIPEA dissolved in DMF. The tail coupling was allowed to proceed for 4.5 hours. The addition of the tail takes longer than amino acid coupling because more DMF is required to dissolve the hydrocarbon tail, and the tail is much larger than an amino acid and more time was allowed to let the tail diffuse into the beads to react with the peptide's N-terminus.
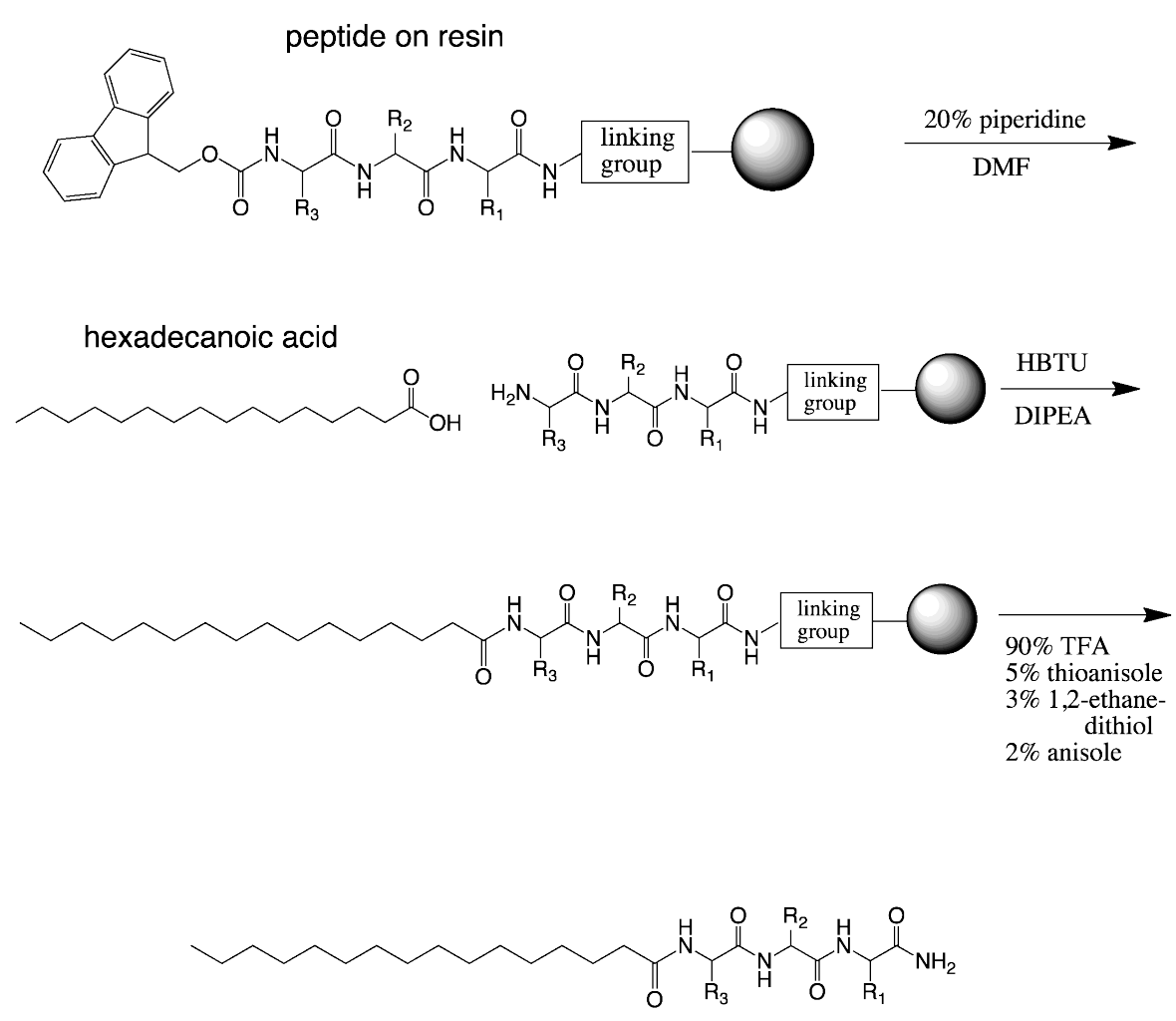

Figure 4-1: Single-tail peptide-amphiphile tail coupling and cleavage from PAL resin bead. First remove Fmoc protecting group. Then couple hexadecanoic acid tail to the peptide on the bead. Last the peptide-amphiphile is cleaved from the bead. Peptide shown is an example tripeptide with side groups $R_{1}, R_{2}$, and $R_{3}$. 
Since PR_g has a large number of side-chain protecting groups, a cleavage solution containing several scavenging agents was used. PR_g was cleaved in $90 \%$ TFA, $5 \%$ thioanisole, $3 \% 1,2$-ethanedithiol, and $2 \%$ anisole. The cleavage reaction was carried out for 4 hours. The cleavage solution was filtered under vacuum to remove the resin beads, and the beads were washed with excess TFA to extract as much product as possible. The TFA solution was rotoevaporated until the product started to precipitate. Ten to twenty times excess cold diethyl ether was added to the solution to precipitate the peptideamphiphile out of solution. The resulting slurry was filtered using a fine sintered glass filter under vacuum. The precipitate was rinsed with excess cold diethyl ether and dissolved in Milli-Q water. The crude product was dried under an air stream and mild heating, dissolved in Milli-Q water again and lyophilized prior to purification.

\subsection{PR_g Purification}

PR_g peptide-amphiphile was purified by reversed phase HPLC as discussed in Section 2.4.4. PR_g purification used a mobile phase gradient of either water and acetonitrile or water and methanol. Acetonitrile provides excellent separation of the peptide-amphiphile from the cleavage reaction impurities, but in 2008-2009 the chemical industry suffered a global shortage of acetonitrile due to the failing automobile industry, Hurricane lke, and the shutdown of an acetonitrile production plants in Beijing for the Olympics. The HPLC method used for PR_g purification had to be adjusted from one using acetonitrile to one using methanol. The stationary phase was a Waters XTerra Prep MS $\mathrm{C}_{18}$ column ( $5 \mu \mathrm{m}$ beads, $125 \AA$ pore size, $10 \times 150 \mathrm{~mm}$ ). A stationary phase with $\mathrm{C}_{18}$ was chosen to increase the retention time of PR_g peptide-amphiphile to enhance separation. As stated in Chapter 2, peptide-amphiphiles with dialkyl tails were separated on a $\mathrm{C}_{4}$ column because of the strong hydrophobic 
interactions between the tails and the stationary phase. Contrarily, PR_g peptide-amphiphile has a single hydrocarbon tail and is significantly less hydrophobic than the dialkyl peptide-amphiphiles. So, a longer alkyl group on the stationary phase was needed to achieve sufficient purification.

The first mobile phase gradient that was developed used $0.1 \%$ TFA in water and $0.1 \%$ TFA in acetonitrile (ACN). Initial runs were performed to determine at what organic concentration PR_g would elute. PR_g crude product was dissolved at a concentration of approximately $1 \mathrm{mg} / \mathrm{mL}$, and $5 \mathrm{~mL}$ were injected into the HPLC. The temperature of the column was maintained at $40^{\circ} \mathrm{C}$, and the solvent flow rate was $4 \mathrm{~mL} / \mathrm{min}$. The first test gradient ran $0 \%$ to $60 \% \mathrm{ACN}$ in 30 minute, which is a rate of change of $2 \% A C N$ per minute. This run indicated that PR_g eluted at approximately 50\% ACN. The gradient was adjusted to slow the rate of change of \%ACN and achieve better separation and the final gradient is shown in Table 4-1. The final gradient ran at $2 \% \mathrm{ACN} / \mathrm{min}$ for 20 minutes, then slowed to $0.5 \% \mathrm{ACN} / \mathrm{min}$ for the next 20 minutes, and then back to $2 \% \mathrm{ACN} / \mathrm{min}$ for the last 5 minutes. The last 10 minutes of the run flushed the column with the initial mobile phase to ready the system for the next run. PR_g eluted at approximately $42 \%$ ACN with this gradient.

Table 4-1: HPLC gradient for purification of PR_g with acetonitrile

\begin{tabular}{|c|c|}
\hline Time (min) & \% Acetonitrile \\
\hline 0 & 0 \\
\hline 20 & 40 \\
\hline 40 & 50 \\
\hline 45 & 60 \\
\hline 45.5 & 0 \\
\hline 55 & 0 \\
\hline
\end{tabular}


When the global shortage of ACN necessitated a change in organic solvent, the HPLC method needed to be reconfigured for purification for a new organic solvent. Methanol with $0.1 \%$ TFA $(\mathrm{MeOH})$ was chosen as the new solvent because it dissolves organic molecules readily, evaporates quickly, and did not require the high-pressure that isopropanol demanded on the system. The column temperature was kept at $40^{\circ} \mathrm{C}$, and the flow rate was $4 \mathrm{~mL} / \mathrm{min}$. Sample volume and concentration was also kept as for the acetonitrile runs. In order to develop a purification method for methanol, an initial run was performed from $0 \%$ to $100 \% \mathrm{MeOH}$ at a gradient of $2 \% \mathrm{MeOH} / \mathrm{min}$. PR_g eluted at approximately $73 \% \mathrm{MeOH}$. The method was adjusted to run a gradient quickly through the lower $\mathrm{MeOH}$ concentrations and slow down nearer to the PR_g peak. The final HPLC method is shown in Table 4-2. The gradient was run at $4 \% \mathrm{MeOH} / \mathrm{min}$ for 15 minutes, then the gradient slowed to $1 \% \mathrm{MeOH} / \mathrm{min}$ for 20 minutes, and then sped back to $4 \% \mathrm{MeOH} / \mathrm{min}$ for 5 minutes before returning to the initial solvent composition to prepare for another run. PR_g eluted at $23 \mathrm{~min}$, which equates to $68 \% \mathrm{MeOH}$.

Table 4-2: HPLC gradient for purification of PR_g with methanol

\begin{tabular}{|c|c|}
\hline Time (min) & \% Methanol \\
\hline 0 & 0 \\
\hline 15 & 60 \\
\hline 35 & 80 \\
\hline 40 & 100 \\
\hline 40.5 & 0 \\
\hline 50 & 0 \\
\hline
\end{tabular}

Figure 4-2 shows a chromatogram of a crude PR_g injection using the $0.1 \%$ TFA in $\mathrm{MeOH}$ as the organic mobile phase. Analysis of the area under the 
peaks shows that the crude solution was $\sim 64 \%$ PR_g. The PR_g peak was collected, dried under air, redissolved in Milli-Q water, and lyophilized. The lyophilized powder is frozen until needed. To verify sufficient purification, purified PR_g was run through the HPLC (Figure 4-3). Integration under the peaks on the chromatogram reveals that the purified product was $97.8 \%$ pure.

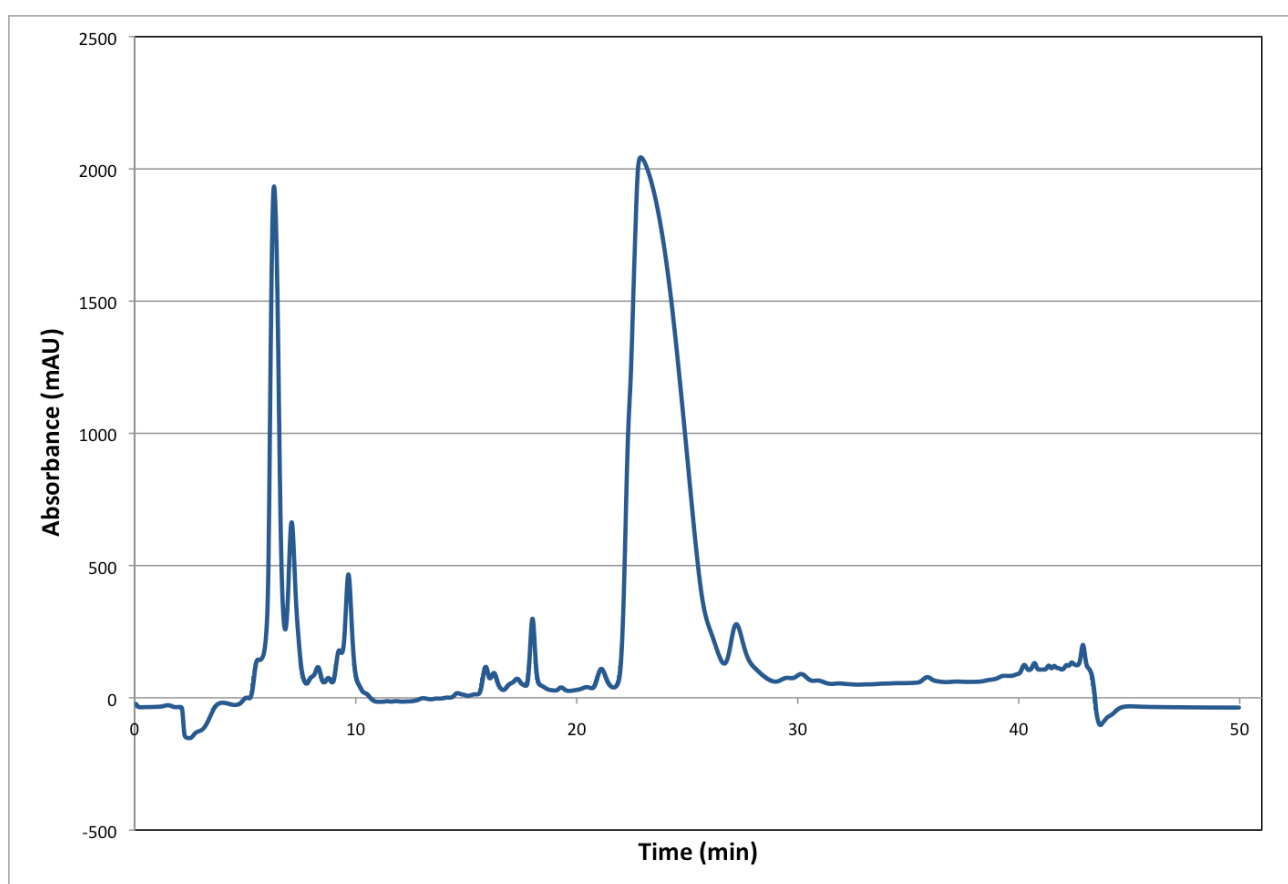

Figure 4-2: Crude PR_g HPLC chromatogram. PR_g elutes at 23 minutes. 


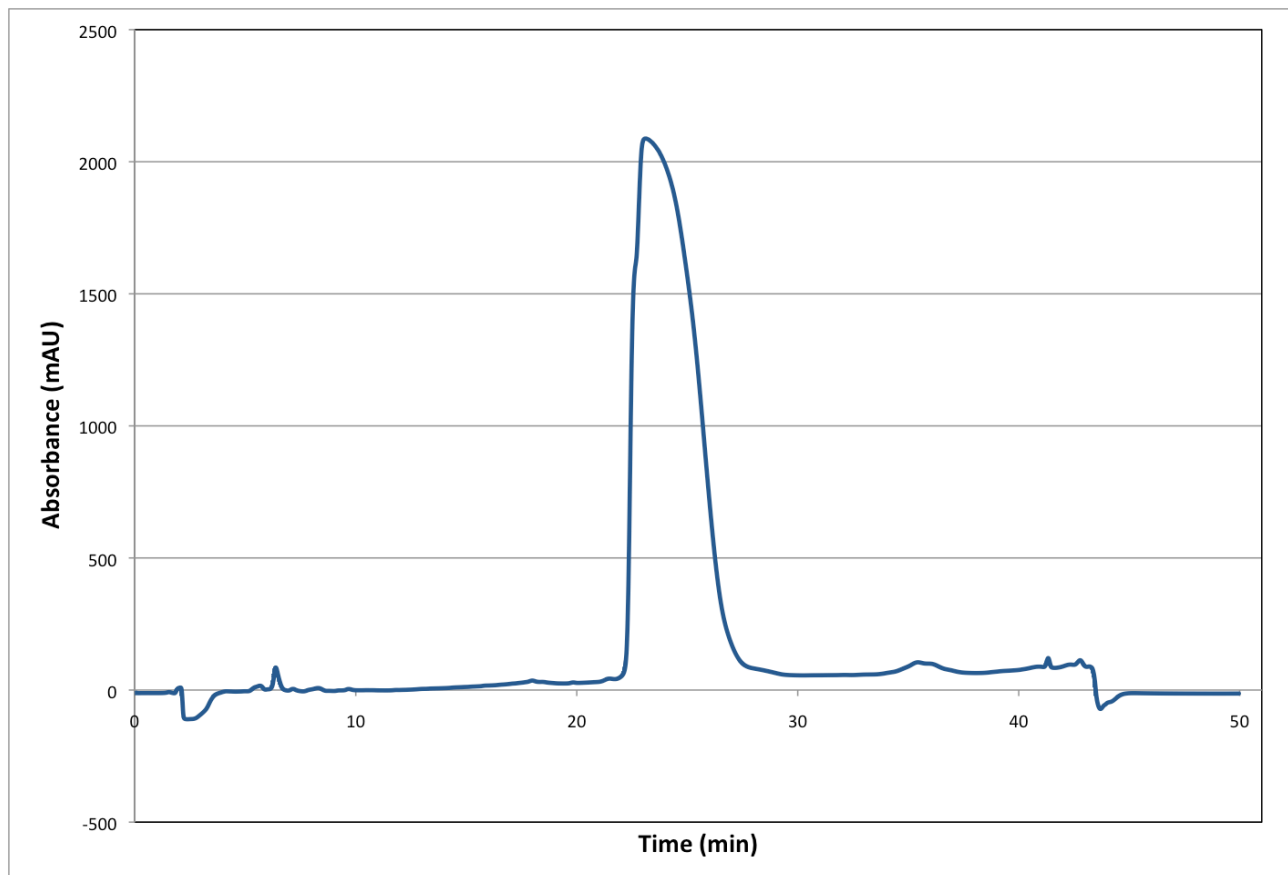

Figure 4-3: Pure PR_g HPLC chromatogram. Integration reveals the product is $97.8 \%$ pure.

\subsection{Molecular Characterization}

\subsubsection{Mass Spectroscopy}

PR_g peptide-amphiphile synthesis was verified using electrospray ionization mass spectroscopy (ESI-MS). ESI-MS is another "soft" mass spectroscopy technique and is used as an alternative to MALDI. MALDI requires that the sample is deposited on a solid substrate; whereas, ESI-MS ionizes a liquid sample for mass analysis. In ESI-MS, the sample is dissolved in a mixture of water and volatile organic solvent, such as methanol. Then it is injected into the system and the liquid stream is exposed to a high voltage producing a fine aerosol and introducing charges into the system. The aerosol droplets evaporate and collapse into smaller and smaller droplets, the charges attach to the analytes, and the charged analytes enter the gas phase. The charged particles then fly through the flight tube and hit the detector, which detects the mass-to-charge $(\mathrm{m} / \mathrm{z})$ ratio. The charge state of peaks on the ESI-MS spectrum 
is determined by examining the isotope peaks. If the isotope peaks differ by $1 \mathrm{~m} / \mathrm{z}$ unit, the charge is +1 . If the isotope peaks differ by $0.5 \mathrm{~m} / \mathrm{z}$ units, the charge is +2 .

PR_g samples were analyzed using a Bruker Biotof II reflectron ESI-MS. Samples were either taken directly from HPLC fractions or dissolved in $50 \%$ $\mathrm{MeOH}, 50 \%$ water solutions and injected into the ESI-MS via syringe pump.

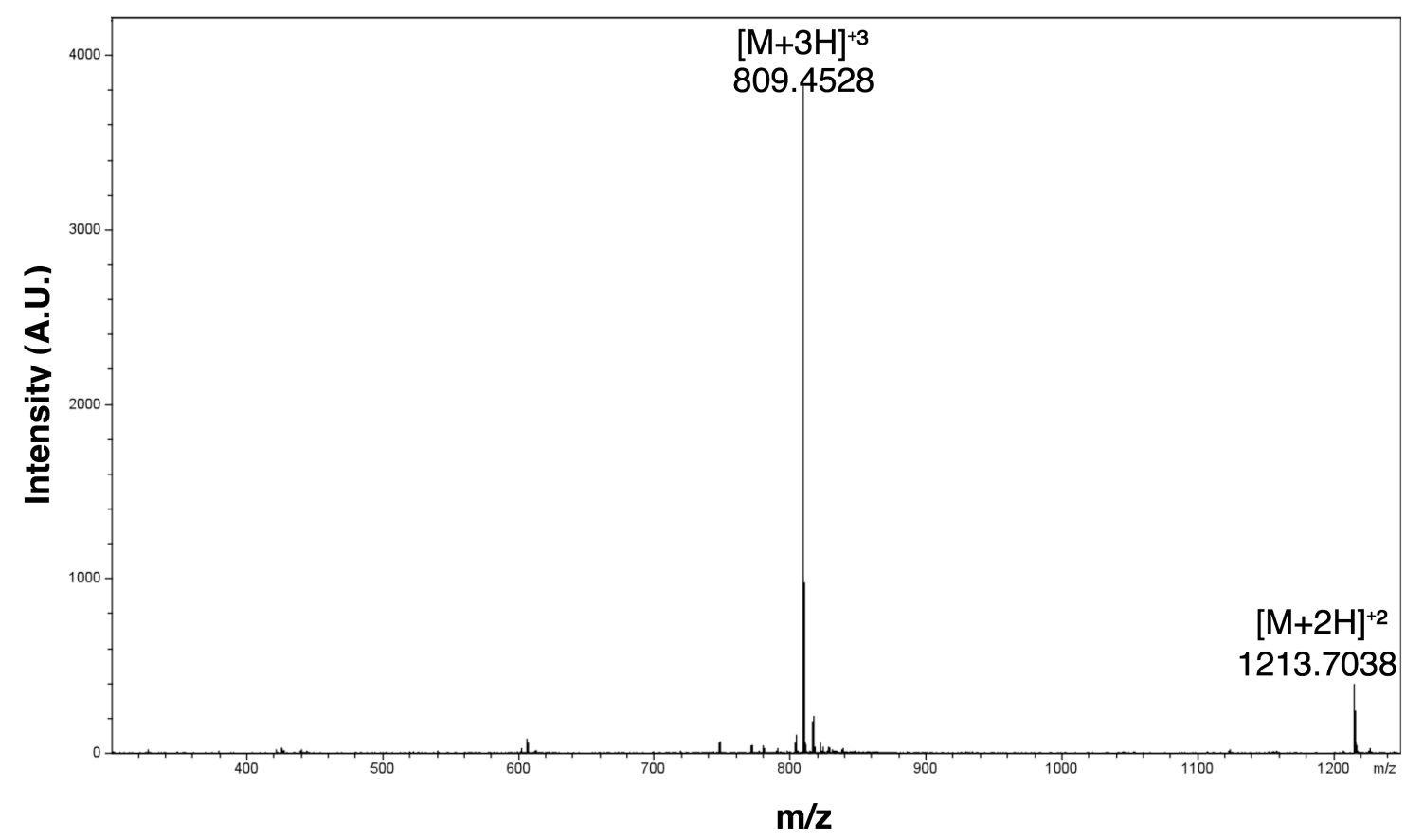

Figure 4-4: ESI spectrum of pure PR_g. The spectrum shows both $a+2$ and +3 charge state for PR_g.

The PR_g spectrum shows two major base peaks (Figure 4-4). The peak at 809.4528 has a charge state of +3 and the peak at 1213.7083 has a charge state of +2. These give experimental masses of 2425.36 and 2425.41, respectively. The theoretical mass of PR_g is $2425.5 \mathrm{Da}$, which falls within the experimental error of the mass spectrometer and confirms the synthesis of PR_g peptide-amphiphile. ESI-MS allows us to examine the presence of some impurities or PR_g derivatives that are found in the purified sample. 
Figure 4-5 takes a closer look at the $\mathrm{m} / \mathrm{z}$ region from $720-900$ and identifies the peaks. Hydrogen is the most common ion found in ESI-MS, but other cations can form adducts. PR_g's spectrum shows both sodium and potassium adducts. Solid phase peptide synthesis increases yields over solution phase chemistry, but the reactions do not always proceed to $100 \%$ completion. This results in some peptide-amphiphiles that may be missing an amino acid. Or conversely, an Fmoc-protecting group may fail to protect from a double addition resulting in an extra amino acid being added to the peptide chain. PR_g's ESIMS spectrum shows examples of both of these. Peaks are seen for PR_g missing a glycine, serine, and asparagine and having an extra glycine and serine. Two peaks not positively identified are PR_g-16 Da and PR_g-183 Da. The loss of $16 \mathrm{Da}$ could be due to a missing oxygen group or a missing $-\mathrm{NH}_{2}$ group. All of the PR_g derivative peaks are very small in comparison to the PR_g mass peak and represent a very small portion of the purified product. Single amino acid deletions or additions are difficult to separate by HPLC unless the amino acid is charged; thus, the purified PR_g contains peptide amphiphiles with missing or added asparagines, serines, or glycines. The pure product is approximately $80 \%$ PR_g, $6 \%$ sodium adduct, $5 \%$ potassium adduct, and $6 \%$ amino acid deletion or addition. 


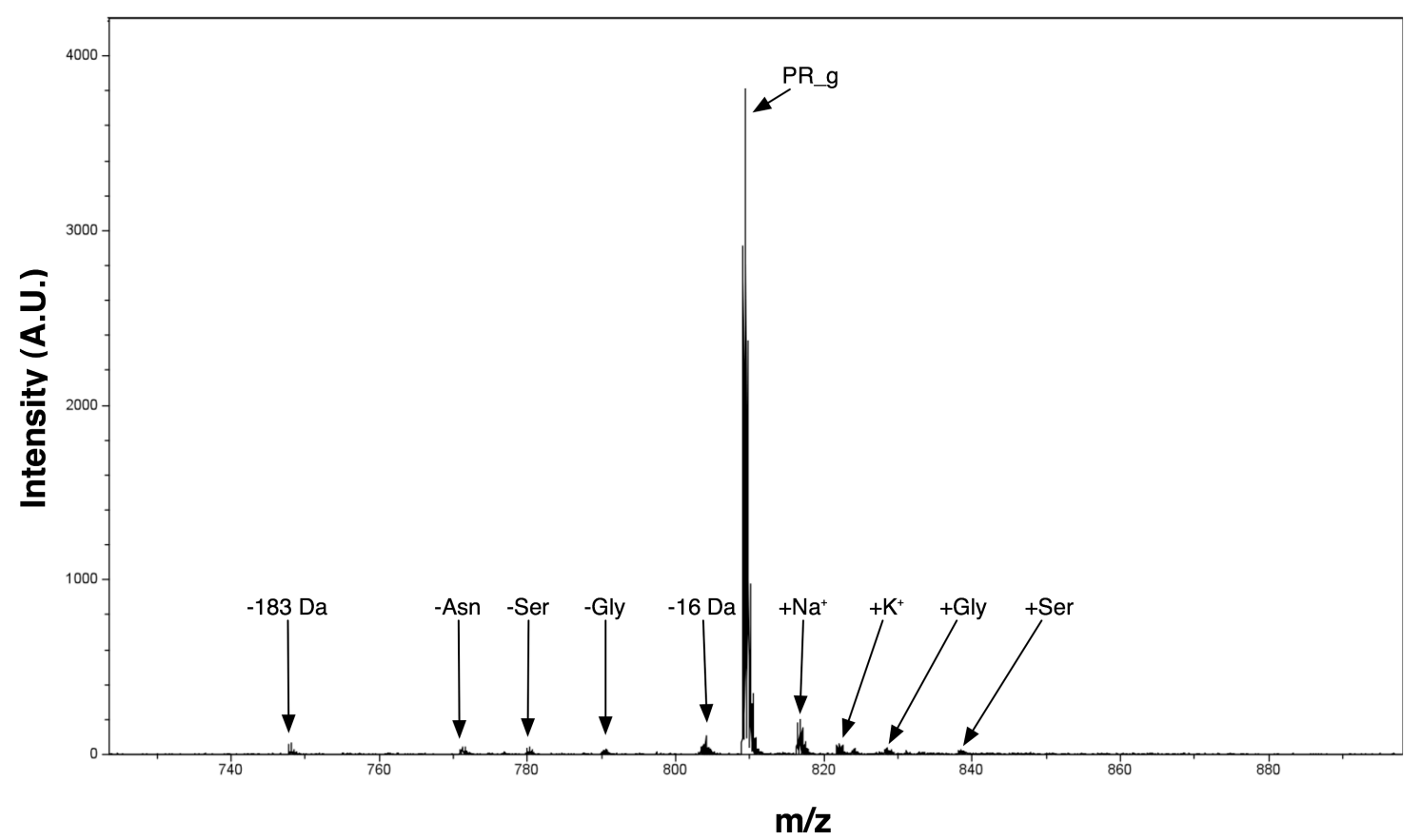

Figure 4-5: ESI spectrum of pure $P R \_g$ zoomed in on the region containing PR_g and PR_g derivatives

\subsubsection{Critical Micelle Concentration}

The critical micelle concentration (CMC) of an amphiphile is the lowest concentration at which the amphiphile forms micelles. Below this concentration the amphiphiles do not associate with each other and exist in solution as free molecules. Once the concentration is raised to the $\mathrm{CMC}$, the amphiphiles spontaneously self-assemble into micellar structures. Increasing the concentration above the CMC results in the creation of more micelles. Micellization induces a change in many solution properties including surface tension, osmotic pressure, and turbidity. ${ }^{194}$ The CMC of PR_g was determined in order to ensure that subsequent experiments characterizing the selfassembled nanofibers were performed at concentrations above the CMC.

One common way of determining the CMC is by the dye solubilization method, which introduces a lipophilic dye into an aqueous solution with the amphiphile. 
The lipophilic dye does not dissolve in the water and can only be solubilized when the amphiphile has formed micelles and the dye can be carried in the micelles' hydrophobic cores. The CMC of PR_g was determined using the lipophilic dye, Nile red. ${ }^{195}$ Nile red is highly fluorescent when it is dissolved in a lipid-rich environment, such as the inside of a micelle and is quenched when it is dissolved in water. Nile red was mixed with varying concentrations of PR_g peptide-amphiphile and the $\mathrm{CMC}$ was determined to be the concentration at which the dye exhibits its characteristic fluorescence.

A solution of $2.5 \mathrm{mg} / \mathrm{mL}$ Nile red in methanol was diluted to $200 \mu \mathrm{M}$ Nile red in $50 \mathrm{mM}$ Tris $-\mathrm{HCl}$ buffer. A total of $12.5 \mu \mathrm{L}$ of this prepared dye solution was added to $12.5 \mu \mathrm{L}$ of PR_g peptide-amphiphile dissolved in Milli-Q water. The resulting mixture was pipetted into a black-walled 96-well plate to make peptideamphiphile concentrations ranging from 0 to $500 \mu \mathrm{M}$. The well plate was wrapped in aluminum foil and left to equilibrate for 18 hours. The fluorescence was measured using a SpectraMAX GeminiXS plate reader (Molecular Devices) with an excitation of $550 \mathrm{~nm}$ and emission of $635 \mathrm{~nm}$.

Figure 4-6 shows the fluorescence intensity of Nile red in solution as a function of the PR_g amphiphile concentration. Above the CMC, the fluorescence intensity increases linearly with increasing PR_g concentration. A linear regression was fit to the data and is shown in the figure as the solid line. The CMC was determined to be the point at which the linear regression intersected the background fluorescent intensity. The background fluorescence intensity was calculated as the average intensity of the lowest prepared concentrations below the $\mathrm{CMC}$ and is indicated in the figure by the dotted line. The figure inset shows a zoomed in area of the intersection indicating the critical micelle concentration. The critical micelle concentration of PR_g was determined to be 
$38 \mu \mathrm{M}$, and all subsequent experiments were performed at a minimum concentration of $100 \mu \mathrm{M}$ to ensure PR_g amphiphile micellization.

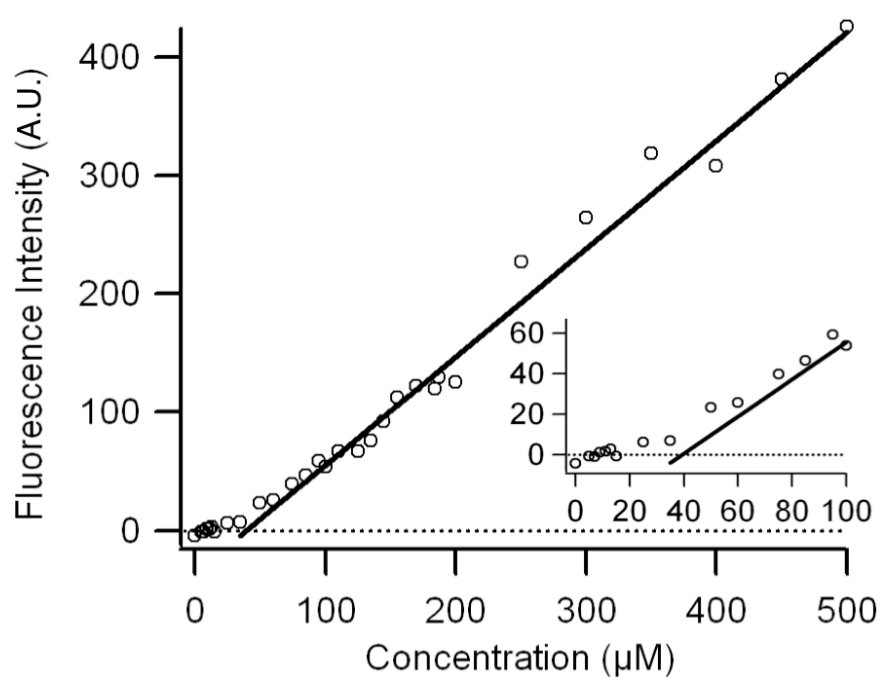

Figure 4-6: Critical micelle concentration of $P R \_g$ determined by solubilization of Nile red in $P R \_g$ micelles. Fluorescence intensity of Nile red dye solubilized in PR_g micelles versus PR_g amphiphile concentration. A linear regression fit (solid line) indicates a CMC of $38 \mu \mathrm{M}$. Background fluorescence was subtracted and is denoted by the dotted line.

\subsubsection{Zeta Potential}

Zeta potential is a physical property of colloidal suspensions. Charged particles in solution affect the distribution of ions in the surrounding solvent. ${ }^{196}$ Counterions tend to concentrate near the surface of the charged particles, and this leads to an electrical double layer surrounding the particles. Ions closest to the charged particle are strongly bound and form a layer called the Stern layer (Figure 4-7). Outside the Stern layer is a more diffuse region where ions are less firmly associated with the particle. If the charged particle were to move, some of the ions in the diffuse layer would move with the particle, and ions further away would stay in the bulk. The boundary between these two regions of the diffuse layer is called the hydrodynamic shear boundary. The zeta potential is defined as the potential at the hydrodynamic shear boundary. The 
magnitude of the zeta potential can be used as an indication of the colloidal system stability. In general, an absolute value of zeta potential greater than approximately $30 \mathrm{mV}$ indicates the system is stable and will not coagulate.

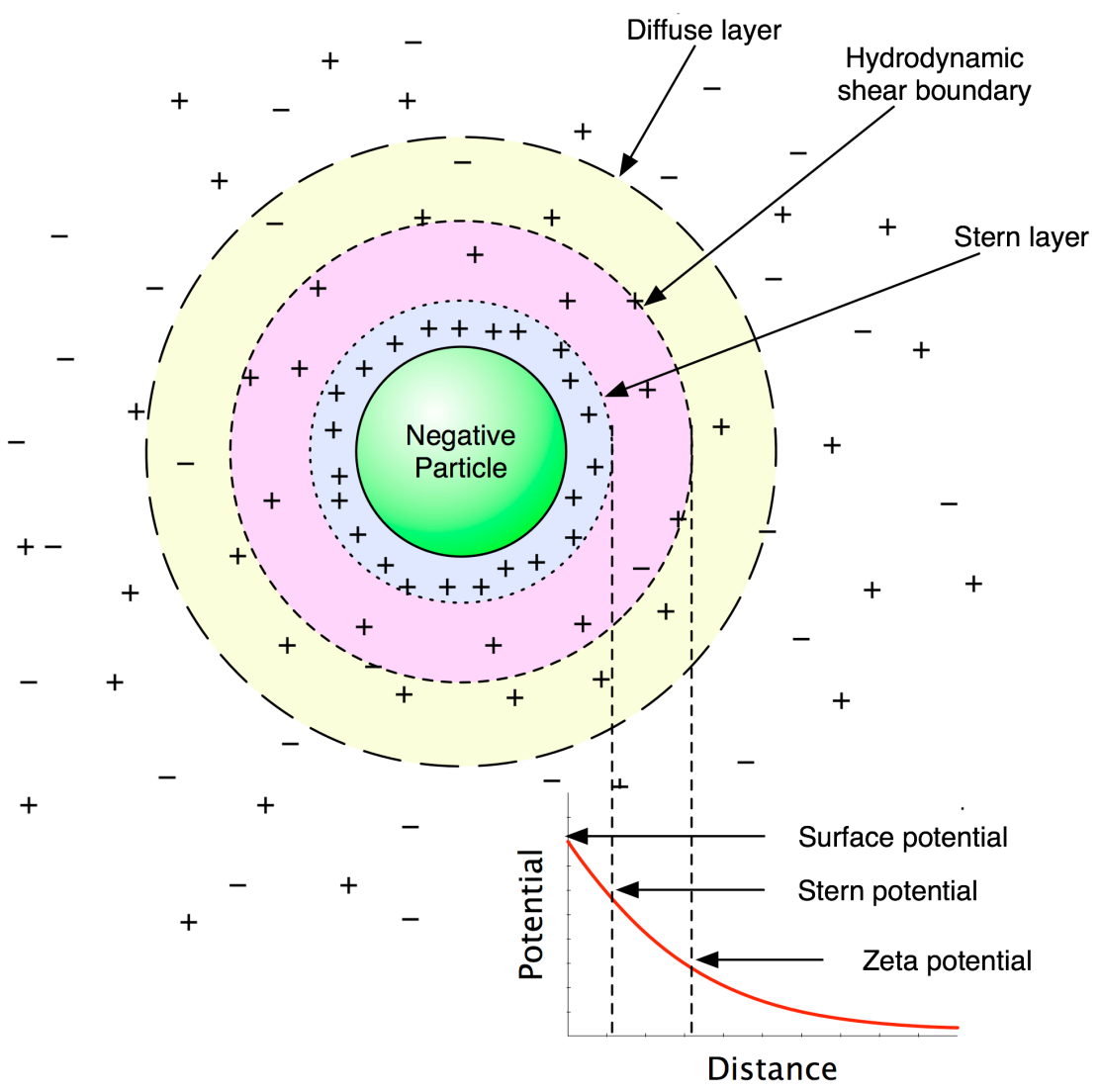

Figure 4-7: Arrangement of ions surrounding a negative particle. The zeta potential is the potential at the hydrodynamic shear boundary. (Adapted from Bioresearch Online ${ }^{197}$ )

PR_g is expected to have a net charge of approximately +1.10 at pH 7. PR_g contains two arginines each of which contributes $a+1$ charge, and the aspartic acid contributes a -1 charge. Histidine's imidazole side chain has a pKa of 6.04 , so in Milli-Q water, some of the histidines will be charged and some will not. ${ }^{193}$

The zeta potential of a $1 \mathrm{mM}$ PR_g peptide-amphiphile solution was measured on a ZetaPALS zeta potential analyzer from Brookhaven Instruments 
Corporation using a clear polystyrene cuvette with a $10 \mathrm{~mm}$ path length. The measurement was taken 20 minutes after the sample was mixed and again after the sample had aged for 48 hours. Data for each run were collected until the relative residual was less than 0.01 . The mobility of the nanofibers was measured and was converted to the zeta potential by the software using the Smoluchowski equation. ${ }^{194}$ The zeta potential was determined to be $38.8 \pm 1.8 \mathrm{mV}$ twenty minutes after preparing the sample, and the measured zeta potential was $42.9 \pm 1.8 \mathrm{mV}$ after the same sample had aged for 48 hours. This indicates that the PR_g peptide-amphiphile is positively charged in Milli-Q water and that its zeta potential does not change significantly with time. The measured zeta potential is consistent with values reported in the literature for similar molecules. ${ }^{182}$

\subsection{Nanofiber Structural Characterization}

\subsubsection{Circular Dichroism Spectroscopy}

Circular dichroism (CD) is a unique property of organic molecules that stems from the differential absorption of right- and left-handed circularly polarized light. ${ }^{198}$ Circularly polarized light is polarized similarly to linearly polarized light except that instead of maintaining one linear direction and possessing an oscillating magnitude, the magnitude of the light remains constant and the direction of the polarization rotates once per wavelength (Figure 4-8). The path of a photon looks like a helix as it travels through space. Left-handed helices are termed left-handed circularly polarized light, and right-handed helices are called right-handed circularly polarized light. Optically active samples absorb left-handed and right-handed circularly polarized light differently due to their asymmetric electrical fields. Achiral molecules possess symmetric electrical fields and do not interact differently with left- and right-handed circularly polarized light. CD spectrometers expose a sample to left- and right-handed 
circularly polarized light and the difference in absorption results in a CD signal. CD spectroscopy is frequently used to determine the secondary structure of organic molecules, such as peptides.

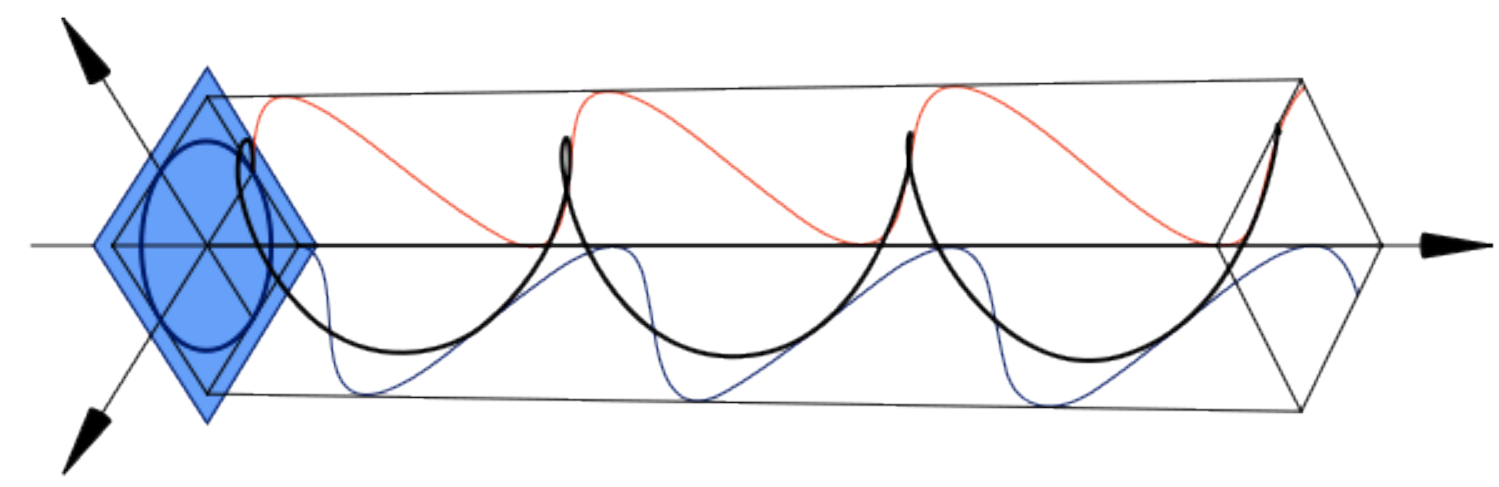

Figure 4-8: Circularly polarized light forms a helix in space as it travels. The light forms a circular pattern on an intersecting plane. (This image was reproduced from Wikipedia and has been released without restrictions into the public domain by its author. ${ }^{199}$ )

The primary optically active bonds in proteins are the peptide bonds in the backbone and any aromatic side chains. Secondary structures such as a-helices and $\beta$-sheets exhibit different orientations of the peptide bonds in the backbone; therefore, secondary structures exhibit characteristic CD spectra. Figure 4-9 gives examples of the CD spectra of peptides exhibiting common secondary structures. ${ }^{200}$ Proteins with a prominent a-helix domains exhibit CD spectra with a negative minimum at $222 \mathrm{~nm}$, a weaker negative minimum at $208 \mathrm{~nm}$, a positive maximum between 190 and $195 \mathrm{~nm}$, and a crossover from positive to negative below $172 \mathrm{~nm} .{ }^{201,} 202$ Proteins that are primarily $\beta$-sheets display a negative minimum between 210 and $220 \mathrm{~nm}$, a positive signal at $\sim 200 \mathrm{~nm}$, a crossover from positive to negative at $185 \mathrm{~nm}$, and a negative minimum around $170-180 \mathrm{~nm}^{201,203}$ 


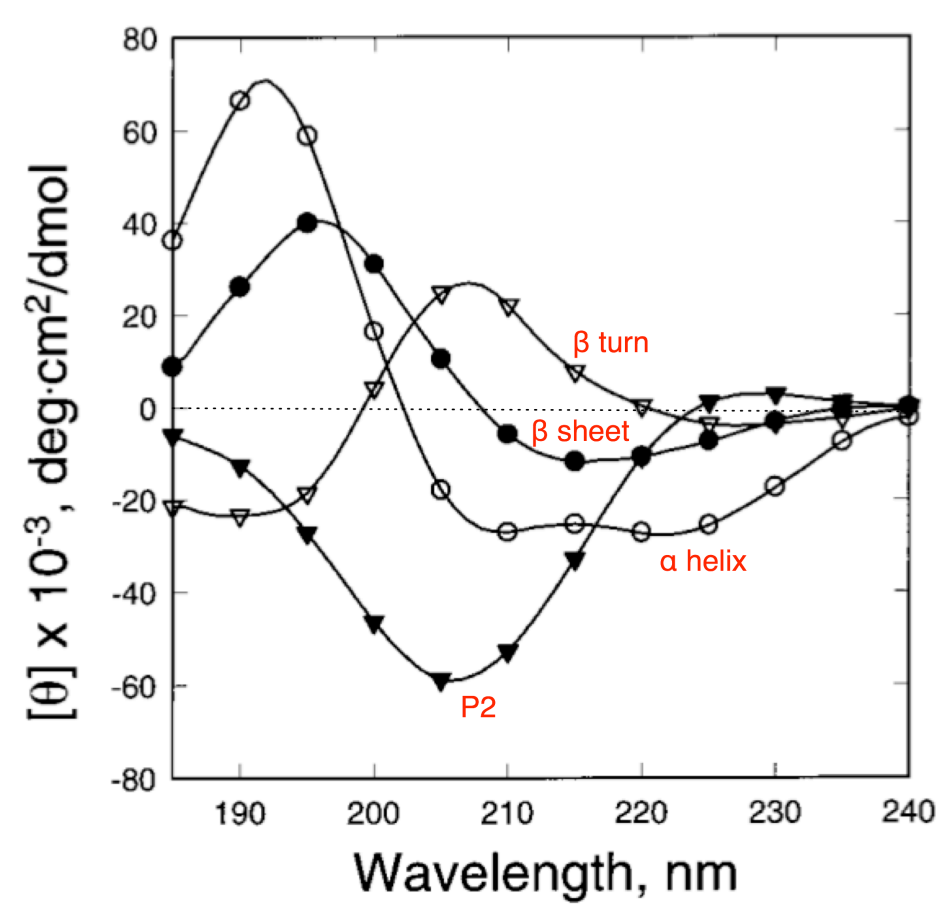

Figure 4-9: CD spectra of peptides exhibiting a-helix (O), $\beta$-sheet $(\bullet), \beta$-turn $(\nabla)$, and P2 conformations $(\nabla)$. (Adapted from reference ${ }^{200}$ )

Many proteins contain both motifs and are termed $\alpha-\beta$ proteins. Two specific types of $\alpha-\beta$ proteins are $\alpha+\beta$ proteins and $\alpha / \beta$ proteins. $\alpha+\beta$ proteins contain separate regions of $\alpha$-helix and $\beta$-sheet. Lysozyme is an example of this protein type ${ }^{204}$ (Figure $4-10$ ). The $\alpha / \beta$ proteins, such as leucine-rich-repeat proteins, contain alternating regions of $\alpha$-helix and $\beta$-sheet. ${ }^{205}$ Both types of $\alpha-\beta$ proteins display similar $C D$ spectra to the $a$-helix proteins in that they show negative minima at approximately 222 and $208 \mathrm{~nm}$ and a positive signal between 190 and $195 \mathrm{~nm}$, but in contrast to they show a crossover from positive to negative above $172 \mathrm{~nm}$. For $\alpha+\beta$ proteins, the minimum around $208 \mathrm{~nm}$ dominates the spectrum and the minimum at $222 \mathrm{~nm}$ is usually a very shallow minimum ${ }^{201}$ (Figure 4-10). 


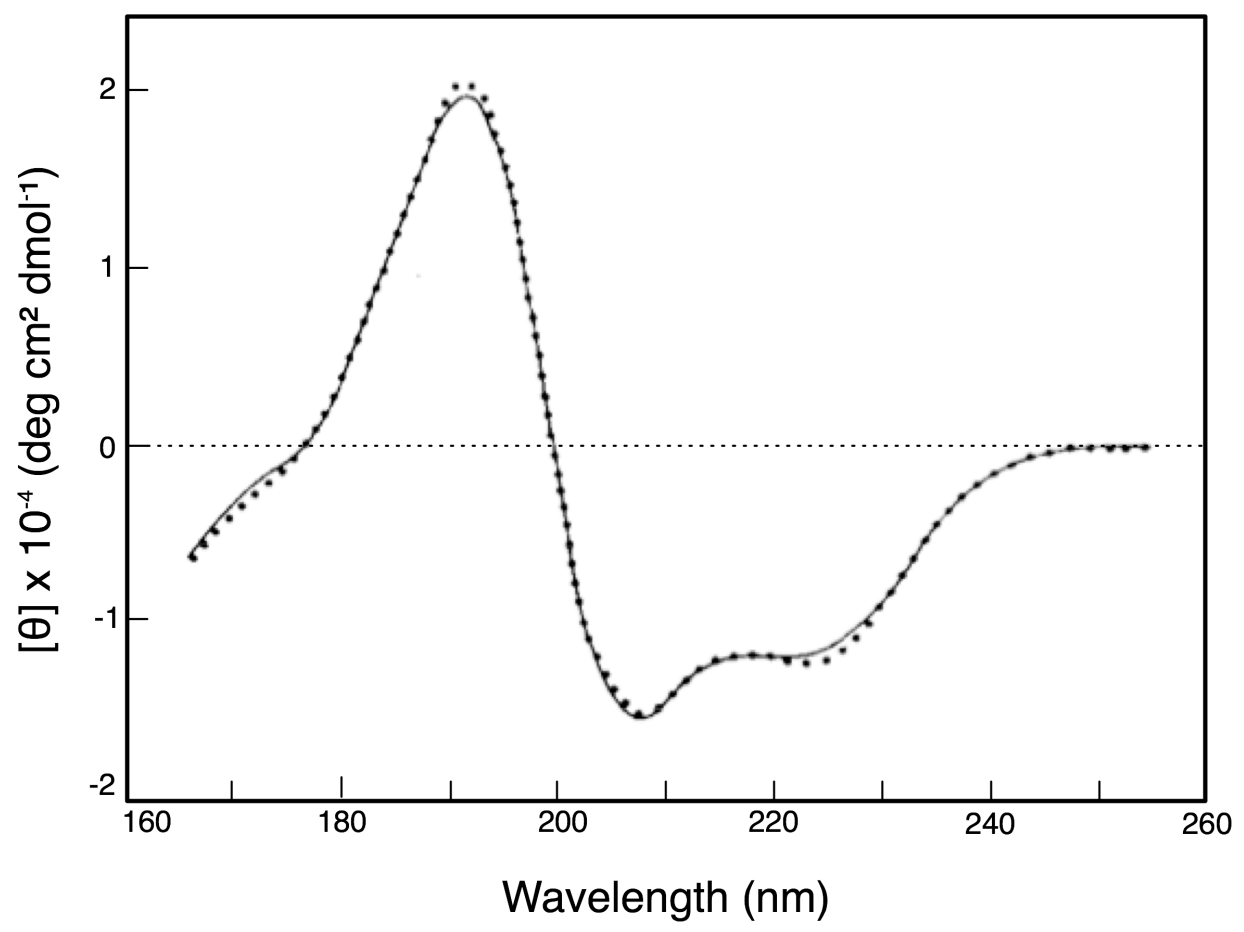

Figure 4-10: CD spectrum of egg white lysozyme. (Adapted from reference. ${ }^{204}$ ) Lysozyme is an example of an $\alpha+\beta$ protein.

Measurements were taken on a JASCO J815 CD spectrapolarimeter at room temperature. Samples were either prepared in Milli-Q water at $100 \mu \mathrm{M}$ or diluted to $100 \mu \mathrm{M}$ from a $1 \mathrm{mM}$ or $10 \mathrm{mM}$ stock solution. The spectra were obtained from 300 to $180 \mathrm{~nm}$ at a bandwidth of $10 \mathrm{~nm}$ and were the average of five individual scans. A background spectrum of Milli-Q water was subtracted from each spectrum. The raw CD signal, $\theta$, was recorded as ellipticity, which has units of mdeg and was corrected for concentration by converting to mean residue ellipticity, $\theta_{\text {MRE }}$ (Equation 4-1). ${ }^{198}$ By historical convention, mean residue ellipticity for proteins is reported in units of $\mathrm{deg} \cdot \mathrm{cm}^{2} / \mathrm{dmol}$ and is a function of the molar concentration, $c$, pathlength, I (reported in centimeters), and the number of amino acids, $N_{a a}$. 
Equation 4-1: Raw CD signal to molar ellipticity for proteins ${ }^{198}$

$\theta_{\text {MRE }}\left(\mathrm{deg} \cdot \mathrm{cm}^{2} / \mathrm{dmol}\right)=\frac{\theta(\mathrm{mdeg})}{10 \cdot c(\mathrm{M}) \cdot I(\mathrm{~cm}) \cdot N_{a a}}$

The secondary structures of PR_g peptide and PR_g peptide-amphiphile were examined with CD spectroscopy (Figure 4-11). Spectra are shown down to $185 \mathrm{~nm}$ below which the water stars to absorb strongly, and the data are no longer accurate. All measurements were performed at $100 \mu \mathrm{M}$. A stock solution of $1 \mathrm{mM}$ PR_g peptide (no hydrocarbon tail) was prepared and then diluted to $100 \mu \mathrm{M}$ immediately prior to measurement. The PR_g peptide shows a minimum at $196 \mathrm{~nm}$. The peptide's CD spectrum is not dependent on the age of the stock solution, and it shows the same spectra after aging the stock solution for 20 days (data not shown). The peptide shows no particular secondary structure and is classified as an unordered sequence. The peptideamphiphile, however, shows a more interesting structure, and the amphiphile's CD spectrum depended upon the age of the stock solution. The CD spectrum of a $100 \mu \mathrm{M}$ sample diluted from a $1 \mathrm{mM}$ stock solution, which was prepared immediately before the CD measurement is similar to the peptide's spectrum (Figure 4-11; $0 \mathrm{hr}$ ). However, when the $1 \mathrm{mM}$ stock solution was prepared and allowed to age for 6 hours before dilution to $100 \mu \mathrm{M}$ for measurement, the spectrum shows a slight shift. The signal at $219 \mathrm{~nm}$ is much stronger and the minimum has shifted right to $198 \mathrm{~nm}$. The stock solution was then allowed to age for 48 hours before dilution to $100 \mu \mathrm{M}$, and the peptide-amphiphile shows a spectrum with a minimum at $203 \mathrm{~nm}$ and a less prominent minimum at $220 \mathrm{~nm}$, resembling the spectrum of an $\alpha+\beta$ protein. Allowing the stock solution to age for 20 days had no further effect on the spectrum shift. The difference in the secondary structure between the peptide and peptide-amphiphile provides further evidence that the tail lends stability to the secondary structure as has been suggested in the literature. ${ }^{86-88}$ 


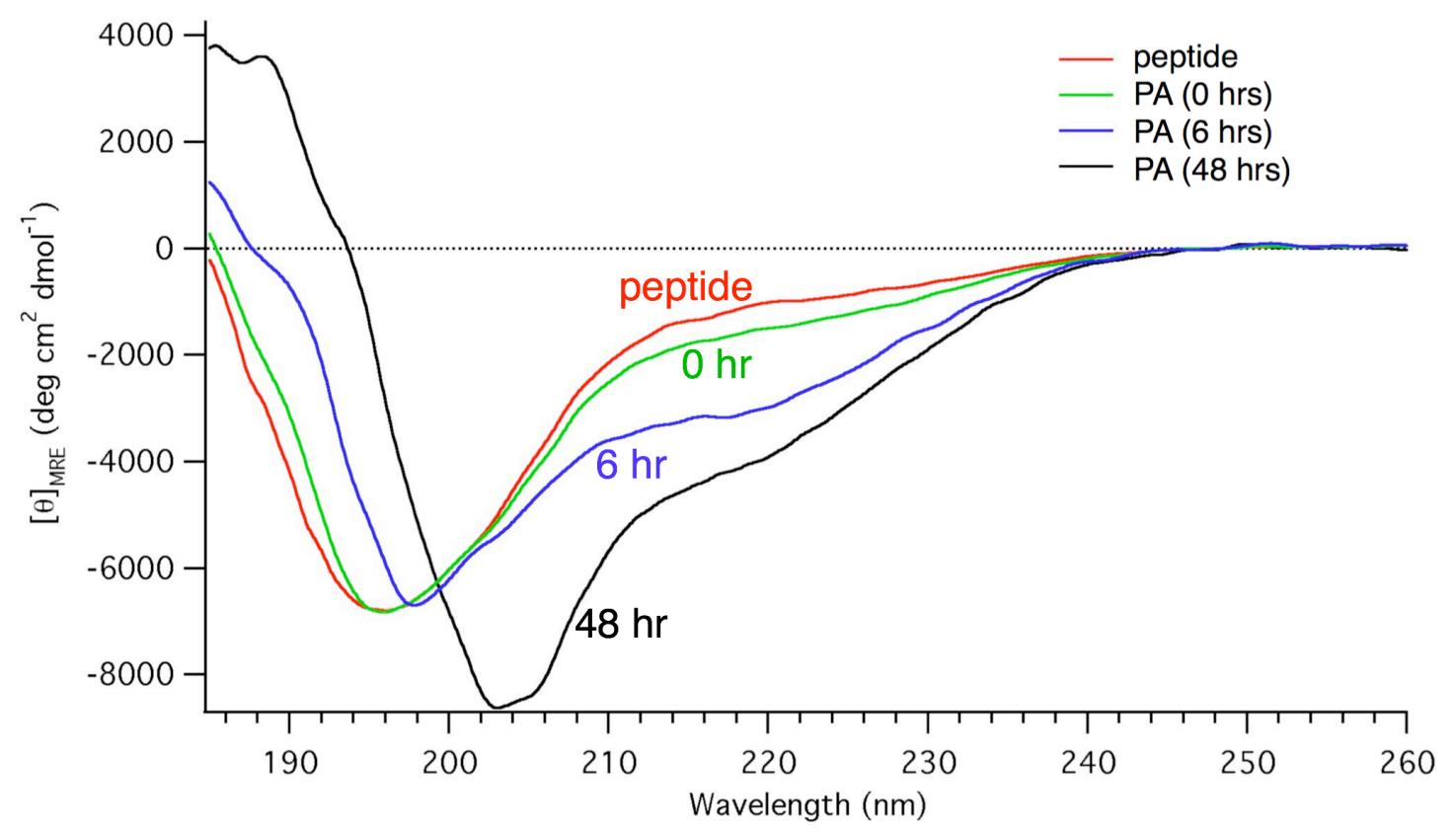

Figure 4-11: CD spectroscopy of PR_g peptide and peptide-amphiphile (PA). The peptide sample was diluted to $100 \mu \mathrm{M}$ from a $1 \mathrm{mM}$ stock solution and shows an unordered structure. A $1 \mathrm{mM}$ stock solution of the PA was prepared and was allowed to age for 0,6 , and 48 hours after which a PA sample was diluted to $100 \mu \mathrm{M}$. CD spectra were taken immediately after dilution. After $48 \mathrm{~h}$, the PA shows a spectrum that resembles more the spectrum of an $\alpha+\beta$ protein. The results are representative of three independent experiments performed on different days. Data are shown from a single experiment.

To test whether the CD spectrum was affected by the concentration of the stock solution, a $10 \mathrm{mM}$ PR_g amphiphile stock solution was prepared and diluted to $100 \mu \mathrm{M}$ at several time points for CD measurement (Figure 4-12). The solution exhibits the unordered secondary structure upon aging of the stock solution for $6 \mathrm{~min}$ and $1 \mathrm{hr}$ before dilution, but after $2 \mathrm{hr}$ the spectrum is similar to the spectrum observed when the $1 \mathrm{mM}$ stock solution has been allowed to age for $48 \mathrm{hrs}$. When the $10 \mathrm{mM}$ solution was allowed to age for $5 \mathrm{hrs}$ prior to dilution, the CD signal intensity strengthened. 


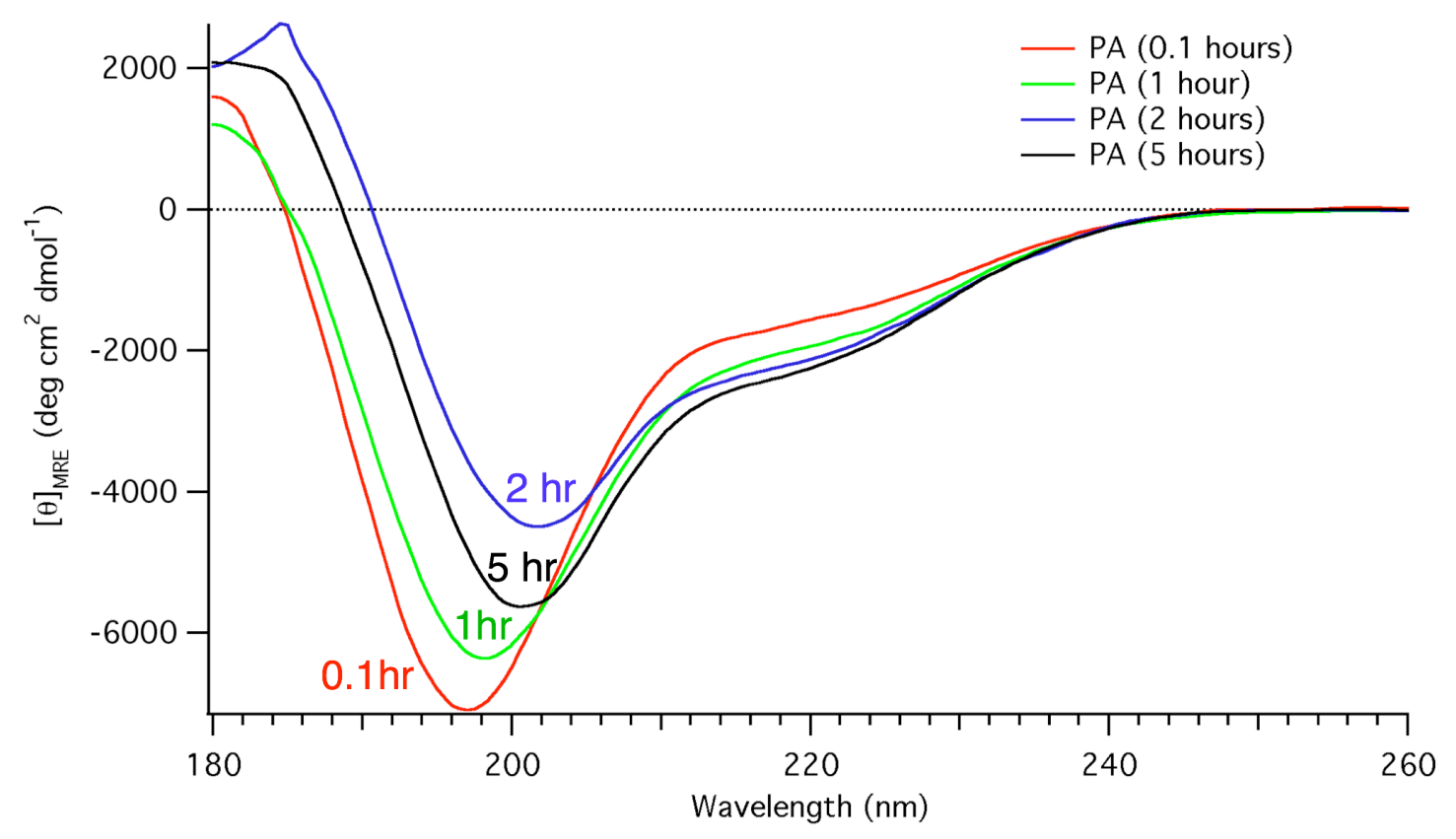

Figure 4-12: CD spectroscopy of a $100 \mu \mathrm{M}$ PR_g amphiphile solution diluted from a $10 \mathrm{mM}$ stock solution. Stock solution was diluted at $0.1,1,2$, and 5 hours after dissolution. At $0.1 \mathrm{hr}$, the CD spectrum shows an unordered secondary structure, and by $2 \mathrm{hr}$ the spectrum resembles that of an $\alpha+\beta$ protein. At $5 \mathrm{hr}$, the intensity of the signal has increased. The results are representative of three independent experiments performed on different days, but data are shown from a single experiment.

These CD spectroscopy results show that the CD spectra of the PR_g peptideamphiphile match the spectra of $\alpha+\beta$ proteins, indicating that the PR_g peptideamphiphile assembled structures contain separate regions of $\alpha$-helix and $\beta$ sheet. This differs from the CD spectra of most other nanofiber-forming peptide-amphiphiles. Previous research shows that nanofiber-forming peptideamphiphiles exhibit predominantly $\beta$-sheet secondary structures after the addition of charge-screening ions or mixing with oppositely charged peptideamphiphiles. ${ }^{170,182}$ One study has reported that peptide-amphiphiles containing some $a$-helical structures at $\mathrm{pH} 10$ in addition to primarily $\beta$-sheet structures. ${ }^{179}$ Prior to the addition of charge screening molecules, the peptide-amphiphiles typically show an unordered secondary structure similar to the structure of PR_g 
peptide. Addition of charge-screening molecules causes the peptideamphiphiles to exhibit a primarily $\beta$-sheet structure. In our case, however, the secondary structure forms over time without adding any charge-screening agents.

\subsubsection{Cryo-Transmission Electron Microscopy}

Cryo-transmission electron microscopy (cryo-TEM) is an electron microscopy technique used to observe aqueous samples. In TEM, a beam of electrons is transmitted through a thin sample, and contrast in a TEM image is dependent on the absorption of electrons due to sample composition and thickness. Electron microscopy is run in a vacuum, which requires that samples be dried before imaging. The drying process can introduce artifacts into samples, and with biological samples, such as proteins, bound water is responsible for much of the formation of the native structure. The nanofiber structure formed by peptide-amphiphiles is highly influenced by the surrounding aqueous media, and drying the samples would likely change the structure of the nanofiber. In cryo-TEM, the sample is plunged into a cryogenic liquid, such as liquid propane or ethane, which vitrifies the liquid and allows the sample to be viewed in its native environment.

PR_g samples were prepared for cryo-TEM in a controlled environment vitrification system. A thin film of PR_g was created by pipetting $10 \mu \mathrm{L}$ of the sample solution onto a copper TEM grid coated with a polymer film (Ted Pella, Inc.). The sample was blotted to remove the excess sample and create a thin film. The grid was quickly plunged into liquid ethane to vitrify the water then stored in liquid nitrogen until imaged. Images were collected by Todd Pangburn and Dr. Wei Fan using a JEOL JEM-1210 transmission electron microscopy operating at $120 \mathrm{kV}$, and the images were captured with a Gatan 724 multiscan 
digital camera. To prevent sample melting during imaging, the temperature in the transmission electron microscope was maintained at $-179^{\circ} \mathrm{C}$.

Cryo-TEM was performed on PR_g peptide-amphiphile solutions at $100 \mu \mathrm{M}$ and $1 \mathrm{mM}$ (Figure 4-13). A $100 \mu \mathrm{M}$ PR_g peptide-amphiphile solution was prepared by diluting a 2-day-old $1 \mathrm{mM}$ stock solution with Milli-Q water. Figure 4-13A shows several single nanofibers forming a "W" shape. The nanofibers all have a diameter of approximately $10 \mathrm{~nm}$ and lengths on the order of microns.
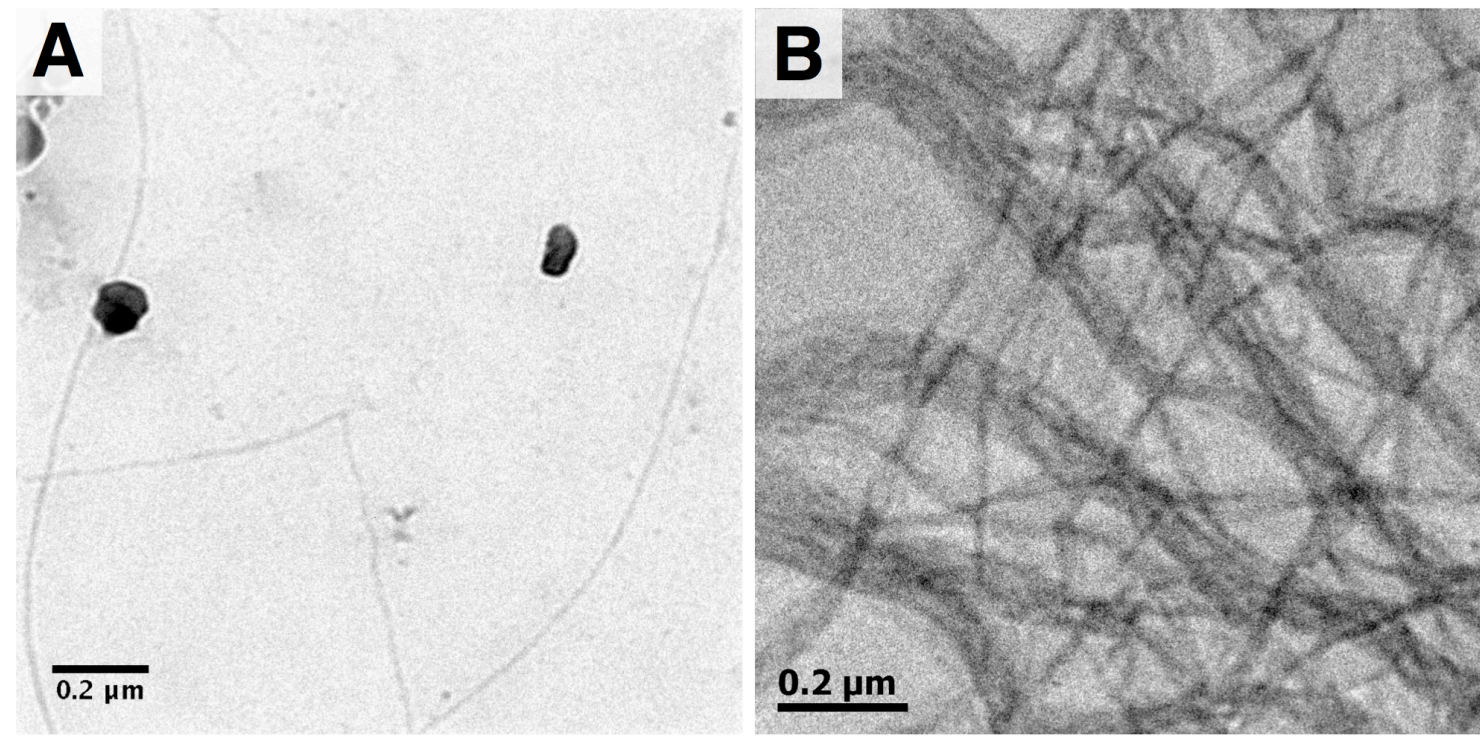

Figure 4-13: Cryo-TEM images of PR_g nanofibers. (a) $100 \mu \mathrm{M}$ sample of PR_g amphiphile showing several nanofibers in a "W" shape. The diameter of the fibers is approximately $10 \mathrm{~nm}$. (b) $1 \mathrm{mM}$ PR_g amphiphile. Fibers appear to be interacting in bundles and show some branching.

The theoretical length of the peptide-amphiphile molecule depends upon the secondary structure of the peptide headgroup. If the peptide headgroup exhibits an unordered structure, the headgroup length measures $3.7 \AA$ /amino acid. 98,118 If the hydrocarbon tail is in a fully extended conformation, it is approximately $2 \mathrm{~nm}$ long, assuming a carbon-carbon bond length of $1.54 \AA$ and a bond angle of $109^{\circ}$. This leads to an overall peptide-amphiphile length of 
$\sim 11.25 \mathrm{~nm}$, and the nanofibers would have a diameter of approximately $22.5 \mathrm{~nm}$. If the peptide headgroup adopted an all- $\beta$-sheet conformation, the headgroup would measure $3.5 \AA$ A/amino acid, ${ }^{3}$ which corresponds to a peptideamphiphile length of $\sim 10.75 \mathrm{~nm}$. Assuming the peptide headgroup adopted an a-helical conformation with a length of $1.5 \AA$ Aamino acid, the entire amphiphile would measure $\sim 5.75 \mathrm{~nm}$. Thus, the theoretical length of the peptideamphiphile is estimated to be between 5.75 and $11.25 \mathrm{~nm}$, and the selfassembled nanofibers would have a diameter between 11.5 and $22.5 \mathrm{~nm}$. Accounting for experimental limitations, the $10 \mathrm{~nm}$ diameter measured by cryoTEM falls within the smaller end of the theoretical expectations and suggests that the peptide headgroup may be tightly coiled or the headgroup may be tilted. The measured diameter of the nanofibers agrees with the diameters of other peptide-amphiphile nanofibers reported in literature. ${ }^{103,171,178-180}$

Another cryo-TEM grid was prepared with a 2-day-old $1 \mathrm{mM}$ solution (Figure 4-13B). At this higher concentration, PR_g forms nanofibers that bundle together. These bundles branch and entangle with other nearby bundles. The bundles may be stabilized by neighboring nanofibers within these bundles forming non-covalent cross-links, such as hydrogen bonds. The combination of extensive hydrogen bonding and the witnessed branching and entangling behavior could give rise to the gelation of the PR_g amphiphile at higher concentrations. The diameter of the bundled nanofibers was observed to be up to $0.2 \mu \mathrm{m}$ thick and many micrometers long. The images of the $1 \mathrm{mM}$ samples show some single nanofibers with diameters of $\sim 10 \mathrm{~nm}$ that are not assembled into the larger bundles, indicating that the bundles are likely composed of single interacting nanofibers and are not larger aggregates of PR_g. Cryo-TEM at higher concentrations is challenging due to difficulties associated with sample preparation. Higher concentrations of peptide-amphiphile solution possess a 
higher viscosity, which in turn makes it more difficult to blot the solution into the thin film necessary for TEM.

\subsubsection{Small-Angle Neutron Scattering}

Small-angle neutron scattering (SANS) is a scattering technique similar to small-angle X-ray scattering and light scattering used to give information about the size, shape, and material properties of a sample. Unlike X-ray and light scattering, which both use electromagnetic radiation, SANS utilizes a beam of neutrons. Neutron radiation covers the wavelength range from approximately 0.1-3 nm, which is similar to the range of $0.01-0.5 \mathrm{~nm}$ covered by X-rays and is significantly smaller than the wavelengths of visible light $(400-700 \mathrm{~nm})$. The scale covered by neutrons and X-rays make them ideal for studying very small length scales such as bond lengths, which are on the order of $0.1 \mathrm{~nm}$. However, a neutron carries far less energy than an X-ray photon does, which is advantageous when working with biological or other sensitive samples. Also, neutron radiation is very penetrating, and samples several centimeters thick can be probed. Neutron scatter does have some drawbacks though. The primary limitation is that neutron sources are scarce. Generating a neutron beam requires either a nuclear reactor or a particle accelerator that collides protons into something to generate neutrons. According to the Center for Neutron Research at the National Institute of Standards and Technology (NIST), only 24 neutron sources worldwide are available for neutron scattering experiments.

In a SANS experiment, neutrons are produced in a nuclear reactor and "cold" neutrons are selected to send along a beam guide toward the SANS instrument. Cold neutrons are neutrons of lower energy than that of thermal neutrons. The neutrons are filtered by a rotating component that selects neutrons of a certain velocity. The neutron beam is then collimated along a pre-sample flight path. The beam passes through the sample, which scatters some of the neutrons. 
The unscattered neutrons are absorbed by a beam stop and the scattered neutrons hit the detector resulting in the data signal (Figure 4-14). The data is then circularly averaged to produce an intensity plot.

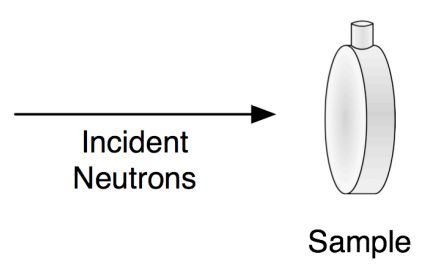

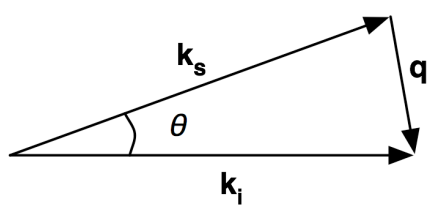

.
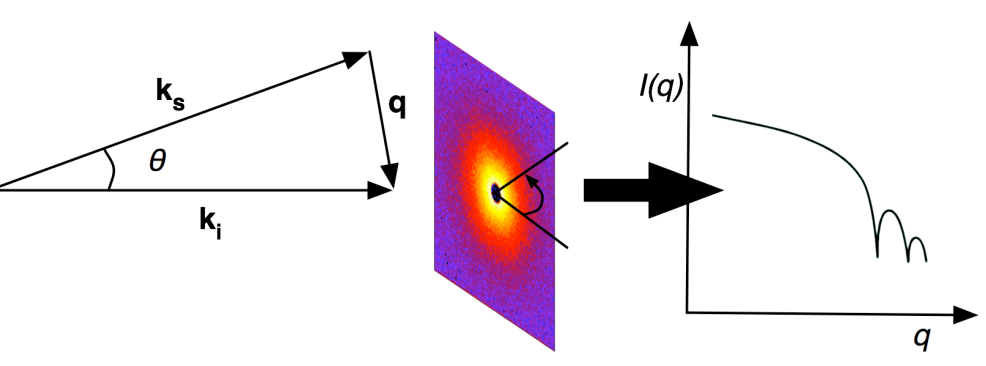

Detector

Figure 4-14: SANS sample scattering schematic

Light scattering is caused by local variations in refractive index or polarizability of a sample, and X-ray scattering is due to local variations in electron density in a sample. Neutrons are scattered by atomic nuclei and are highly sensitive to variations in nuclei type. Contrast in SANS experiments is determined by differences in scattering length density $(S L D)$. The scattering length density of a sample is calculated by Equation $4-2$, where $b_{c}$ is the coherent neutron scattering length of each atom in the sample, $\rho$ is the bulk density, $M_{W}$ is the molecular weight, and $N_{A}$ is Avogadro's number. ${ }^{206}$ The coherent scattering length is dependent on the scattering element, and values for several elements of biological interest are given in Table 4-3. ${ }^{207}$ Most atoms have scattering lengths of similar values, but one of the most significant variations is the difference between hydrogen and its isotope deuterium. Therefore, many SANS experiments are conducted in $\mathrm{D}_{2} \mathrm{O}$ to attain a high level of contrast. Contrast matching can also be employed by mixing $\mathrm{D}_{2} \mathrm{O}$ and $\mathrm{H}_{2} \mathrm{O}$ such that the $S L D$ of the solution matches the SLD of part of the sample, for example, the outer shell of a micelle. 
Equation 4-2: Scattering length density (SLD) ${ }^{206}$

$S L D=\sum_{i} b_{c, i} \cdot \frac{\rho N_{A}}{M_{W}}$

Table 4-3: Coherent scattering lengths for selected atoms ${ }^{207}$

\begin{tabular}{|c|c|}
\hline Element & $\boldsymbol{b}_{\boldsymbol{c}}(\mathbf{f m})$ \\
\hline $\mathrm{H}$ & -3.7390 \\
\hline $\mathrm{D}$ & 6.671 \\
\hline $\mathrm{C}$ & 6.6460 \\
\hline $\mathrm{N}$ & 9.36 \\
\hline $\mathrm{O}$ & 5.803 \\
\hline $\mathrm{P}$ & 5.13 \\
\hline $\mathrm{S}$ & 2.847 \\
\hline
\end{tabular}

The intensity of scattering is quantified in terms of the scattering vector, $q$, which is defined as the difference between the incident and scattered wave vectors, $\mathbf{k}_{\mathbf{i}}$ and $\mathbf{k}_{\mathbf{s}}$, respectively (Figure $4-14$ ). The scattering vector is related to the scattering angle, $\theta$, by Equation 4-3. ${ }^{117}$ The scattering vector is expressed in dimensions of inverse length and is an important variable in establishing what size of structural features can be resolved by an experiment.

Equation 4-3: Scattering vector, $q$, as it relates to the angle of scattering, $\theta^{117}$

$q=\frac{4 \pi}{\lambda} \sin \left(\frac{\theta}{2}\right)$

The raw intensity signal is corrected to remove the intensity signals coming from the empty cell, background scattering, sample transmission, and detector response to the neutron beam. The resulting sample scattering intensity, $I(q)$, is related to $q$ by Equation $4-4$, where $P(q)$ is the form factor and $S(q)$ is the 
structure factor. The form factor, $P(q)$, is a function that describes the shape of the scattering body. Please note that for the form given in Equation 4-4, $P(q)$ also contains the contrast term and volume fraction term. The structure factor, $S(q)$, accounts for interference effects between radiation scattered by multiple scattering bodies. In dilute solutions, $S(q)$ approaches unity and $I(q) \approx P(q)$, and the scattering intensity is primarily a function of the shape of the scattering body.

Equation 4-4: Scattering Intensity as a function of $q^{208}$

$I(q)=P(q) S(q)$

Different particle shapes cause different patterns in neutron scattering. Figure 4-15 shows the log of the scattered intensity, $\log (I(q))$, is plotted against the log of the scattering vector, $\log (q)$, for a sphere, rod, and disc. At low $q$, all three shapes exhibit a slope of 0 . This occurs when $q R_{g}>1$ or when $q$ is on the same order as the radius of gyration, $R_{g}$, and is termed the Guinier region. The Guinier region is shaded yellow (or very light grey if printed in black and white) in Figure 4-15. The slope of the intensity before the terminal region can identify the specific sample shape. Spheres only have a slope of 0 before breaking toward the terminal slope. Rod-like objects such as cylinders, elongated twisted ribbons, and nanofibers exhibit a slope of -1 ; whereas, disc-like or lamellar objects have a slope of -2 before breaking toward the terminal region. In the high $q$ regime, a monodisperse system of scatterers will exhibit peaks that decay with a slope between -3 to -4 . Polydisperse systems will still have a slope between -3 to -4 , but will not show any peaks. This region is called the Porod region (light blue or darker grey shading) and gives information about the surface of the material. A slope of -4 is expected when the system has a sharp interface between the particle and surroundings. If the boundary is diffuse, the slope will approach a value of -3 . 


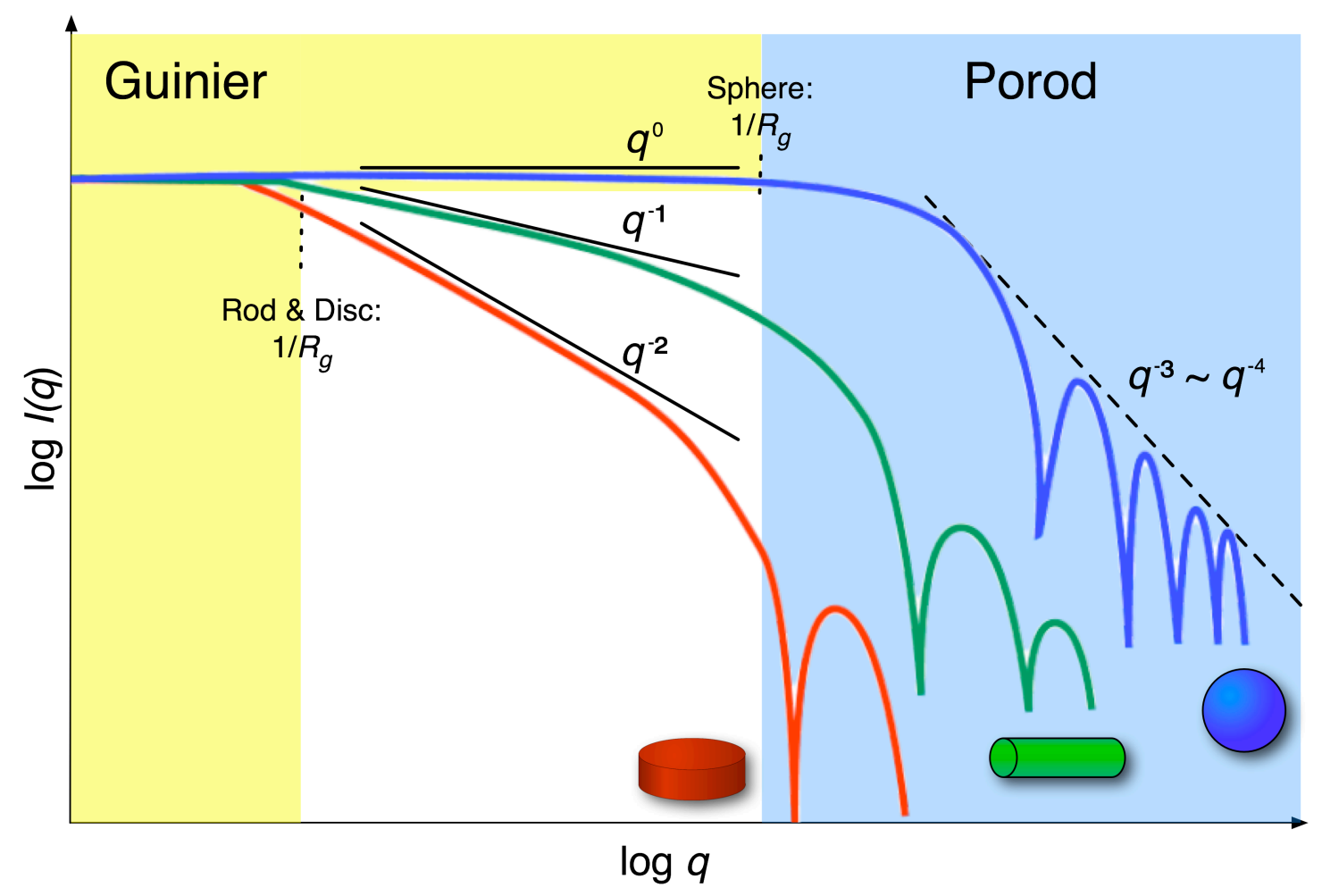

Figure 4-15: SANS scattering intensity as a function of $q$ for a sphere, rod, and disc

SANS measurements were performed at NIST on a $30 \mathrm{~m}$ instrument (NG-7) by Rajiv Taribagil. ${ }^{209}$ This system is capable of producing neutrons with wavelengths of 0.5-2 $\mathrm{nm}$ and detector distances of 1-15.3 $\mathrm{m}$. This allows for a maximum $q$-range of $0.0015-0.6 \AA^{-1}$. Samples of $1 \mathrm{wt} \%(4.12 \mathrm{mM}) \mathrm{PR} \_\mathrm{g}$ and 0.75 wt\% (3.09 mM) PR_g were prepared with $\mathrm{D}_{2} \mathrm{O}$ the night before the experiment. Solutions were pipetted into banjo cells with a path length of $\sim 1 \mathrm{~mm}$ after the overnight equilibration. Sample to detector distances were 1, 3 , and $13 \mathrm{~m}$, which resulted in sampling the $q$-range of $0.003>q(1 / \AA)>0.331$. Neutrons with a wavelength of $\lambda=7 \AA$ with a distribution of $\Delta \lambda / \lambda=0.11$ were used for the SANS measurements. The raw data were reduced to correct for the $\mathrm{D}_{2} \mathrm{O}$ background, the empty cell, background scattering, sample transmission, and detector response to the neutron beam. 


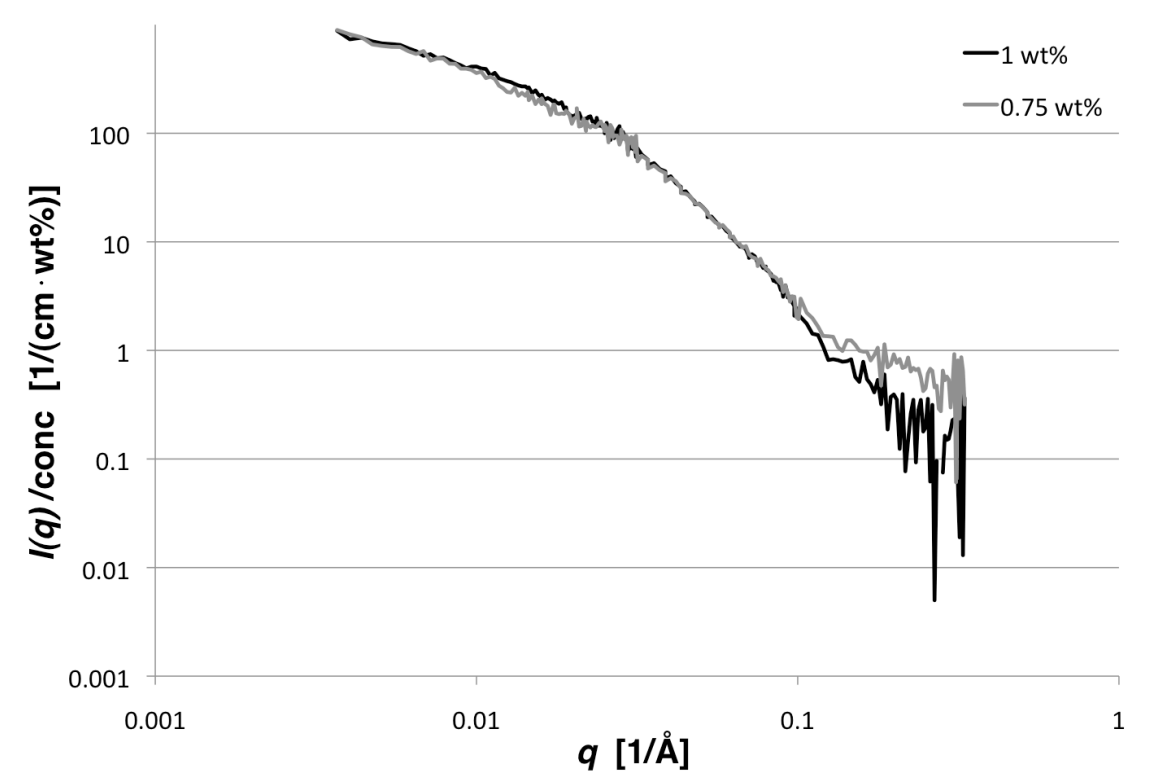

Figure 4-16: SANS data for PR_g peptide-amphiphile solutions at $1 \mathrm{wt} \%$ and $0.75 \mathrm{wt} \%$ normalized with respect to concentration

Two concentrations were used for the SANS experiments: $1 \mathrm{wt} \%$ and $0.75 \mathrm{wt} \%$ of the PR_g peptide-amphiphile. When the intensity $(I(q))$ was normalized with respect to concentration and $I(q)$ was plotted against the scattering vector, $q$, the two sets of data overlapped, indicating that the scattering intensity is proportional to concentration (Figure 4-16). This suggests that the structure of the solution at $1 \mathrm{wt} \%$ is the same structure that is seen at $0.75 \mathrm{wt} \%$. The data shows that the Guinier limit is not reached in the $q$ range $0.003>q(1 / \AA)>$ 0.331. This implies that the radius of gyration for $P R \_g$ is quite large. For cylinders, the radius of gyration, $R_{g}$, is related to the length, $L$, and the radius $R$ of the cylinder by equation 1 . The cryo-TEM images shows that PR_g nanofibers have a diameter $\sim 10 \mathrm{~nm}$ and lengths $>1 \mu \mathrm{m}$. Since $L \gg R$, Equation 4-5 reduces to $R_{g} \approx L /(12)^{1 / 2}$. Assuming that the nanofibers have an $R_{g}$ of $1 \mu \mathrm{m}$, the Guinier limit would be $q=0.0001 \AA^{-1}$, which is outside the range of the instrument used. 
Equation 4-5: Radius of gyration of a cylinder ${ }^{210}$

$R_{g}^{2}=\frac{L^{2}}{12}+\frac{R^{2}}{2}$

The SANS data for $1 \mathrm{wt} \%$ (4.12 mM) PR_g is shown in Figure 4-17. In the $q$ range from 0.0037 to $0.021 \AA^{-1}$, intensity decays with $q^{-1}$. This supports the hypothesis that PR_g forms cylindrical micelles. In the $q$-range from 0.043 to $0.183 \AA^{-1}$, intensity decays with $q^{-3.133}$, which deviates from the expected $q^{-4}$ for particles with sharp interfaces. This could be the result of a diffuse interface between the nanofiber and the solvent, due to a polydispersity in the nanofiber radius, as a result from a different structure factor, or any combination of the above.

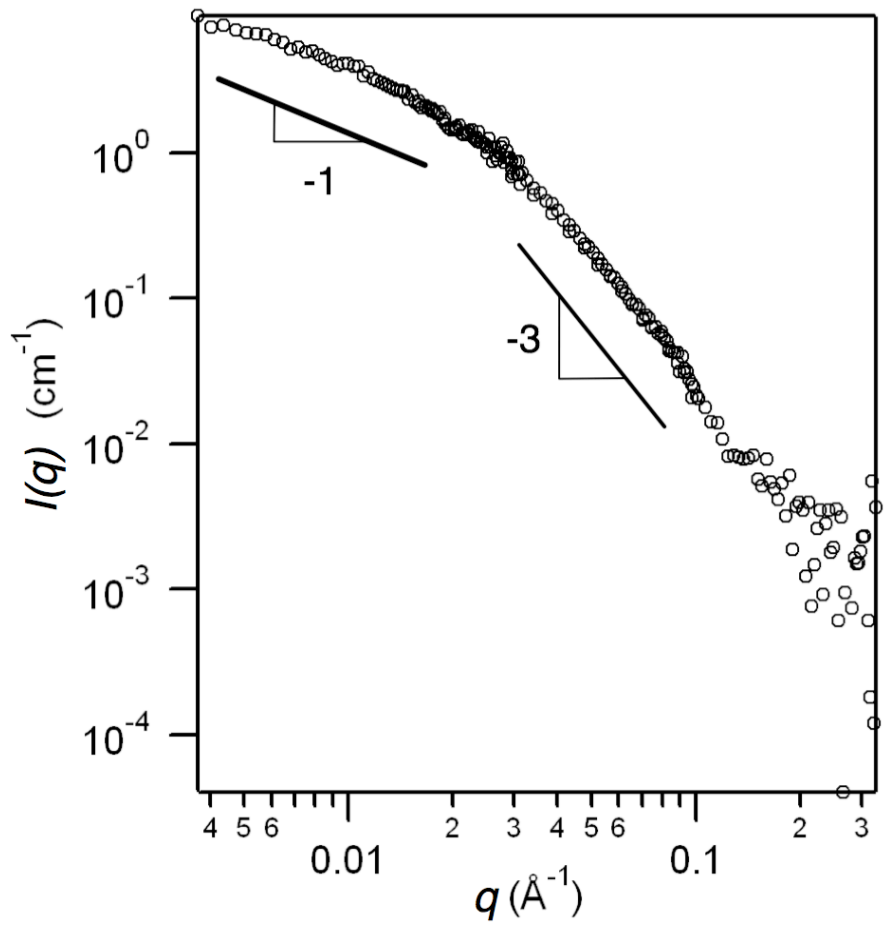

Figure 4-17: SANS data of $1 \mathrm{wt} \%(4.12 \mathrm{mM})$ PR_g amphiphile dissolved in $100 \% \mathrm{D}_{2} \mathrm{O}$, plotted as intensity $(I(q))$ against the scattering vector $(q)$. The intensity first decays as $q^{-1}$. In the Porod scattering region, the intensity decays as $q^{-3.133}$. 


\subsection{PR_g Nanofiber Gel Characterization}

\subsubsection{PR_g Gel Formation}

A gel is a solid, jelly-like substance made of a dilute cross-linked network, which contains a significant amount of fluid. ${ }^{117,211}$ The cross-linked network within the solvent gives the gel structure and allows it to resist flowing. Hydrogels are primarily comprised of water and are frequently used as scaffolds for tissue engineering. PR_g was found to form a self-supporting gel at concentrations greater than $6 \mathrm{mM}$. A $10 \mathrm{mM}$ solution of PR_g peptide-amphiphile was prepared in Milli-Q water. The solution was allowed to sit overnight at room temperature in a small glass vial. Figure 4-18 illustrates the self-supporting nature of the PR_g gel. The contrast of the image has been enhanced to make the gel easier to see. The gel is slightly less opaque than shown in the image.
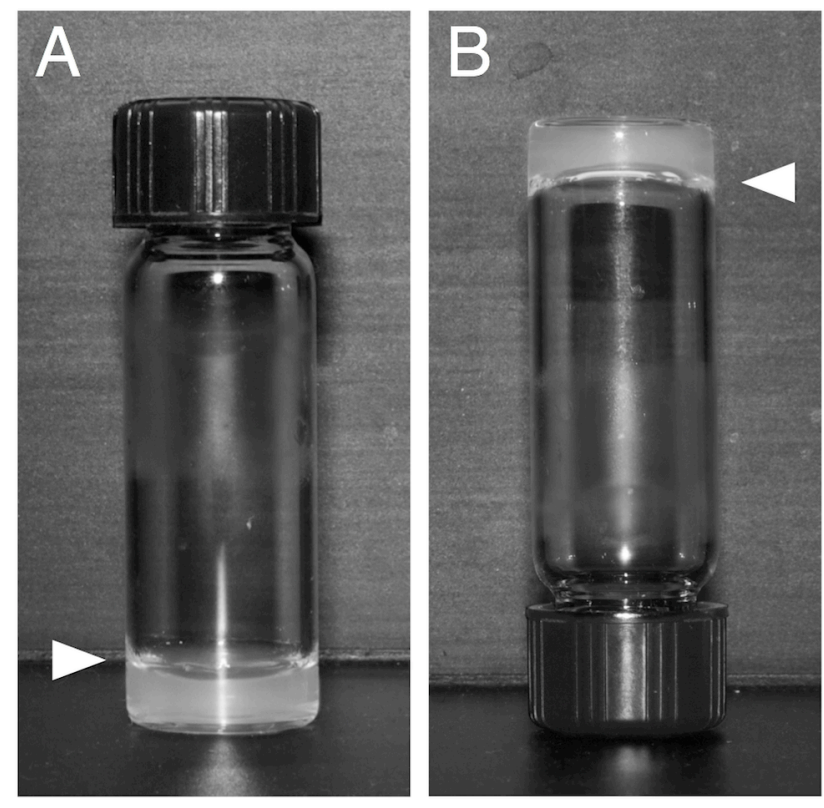

Figure 4-18: PR_g peptide-amphiphile self-supporting gel. PR_g peptide-amphiphile solution (10 $\mathrm{mM}$ ) dissolved in Milli-Q water. The solution was allowed to sit overnight at room temperature. (A) shows gel at the bottom of the vial. (B) shows the same vial tipped upside-down to illustrate that the gel is self-supporting. 


\subsubsection{Rheological Characterization}

As shown in the previous section, when $P R_{-} g$ is dissolved in water at higher concentrations (6 $\mathrm{mM}$ or higher), it appears to form a self-supporting gel. To verify that PR_g actually forms a gel and not just a very viscous liquid, rheology experiments were performed. Rheology is the study of the flow of matter and can be used to determine the mechanics of solid materials. Many soft materials such as hydrogels and polymers are viscoelastic materials meaning that they exhibit behavior that falls in between that of elastic solids and viscous liquids. For this discussion and experiment, only the linear viscoelastic regime will be considered to avoid the complexities involved with non-linear analysis. The shear modulus, $G$, is related to shear stress, $\sigma$, and shear strain, $\gamma$, by Equation $4-6 .{ }^{117}$ In the linear regime, usually found as $\gamma$ approaches $0, G$ is independent of $\gamma$.

Equation 4-6 ${ }^{117}$

$\sigma=G \gamma$

The most common experimental method to characterize the viscoelastic properties of a material is to subject the material of an oscillating strain. When a material is exposed to an oscillating shear strain of the form, $\gamma(t)=\gamma_{0} \sin (\omega t)$, the shear stress response also responds in an oscillatory fashion with the same frequency but with a different amplitude and phase. The phase shift in the stress response can be resolved into components that are $100 \%$ in-phase with the driving oscillation and $100 \%$ out-of-phase (Equation 4-7). ${ }^{117}$

Equation 4-7 ${ }^{117}$

$\sigma(t)=G^{\prime} \gamma_{0} \sin (\omega t)+G^{\prime \prime} \gamma_{0} \cos (\omega t)$ 
$G^{\prime}$ is the in-phase component and is called the elastic or storage modulus. G" is the out-of-phase component and is called the viscous or loss modulus. The relationship between these two components can be used to describe the viscoelasticity of the material. When $G^{\prime} \approx G^{\prime \prime}$ the material is said to be viscoelastic. When the $G^{\prime} \gg G^{\prime \prime}$, the material is more solid-like, and for $G^{\prime \prime} \gg G^{\prime}$, the material behaves more liquid-like.

Rheological properties were measured using an AR-G2 apparatus from TA Instruments. Time sweep experiments were performed using a $40 \mathrm{~mm}$ parallel plate. A $10 \mathrm{mM}$ solution of PR_g was prepared and pipetted onto a Peltier plate maintained at a temperature of $25^{\circ} \mathrm{C}$. The top parallel plate was lowered to make contact with the sample, and the gap was decreased until the sample completely filled the gap. The sample was allowed to gel and a strain sweep was performed to determine the linear viscoelastic region (Figure 4-19). The strain started at $0.1 \%$ was allowed to increase until the sample started to leave the linear regime. The data shows that the relationship between stress and $\%$ strain is linear until approximately $1.5 \%$ strain. All subsequent experiments were performed at $\%$ strains less than or equal to $1 \%$ strain to ensure that the material's performance was in the linear viscoelastic regime. 


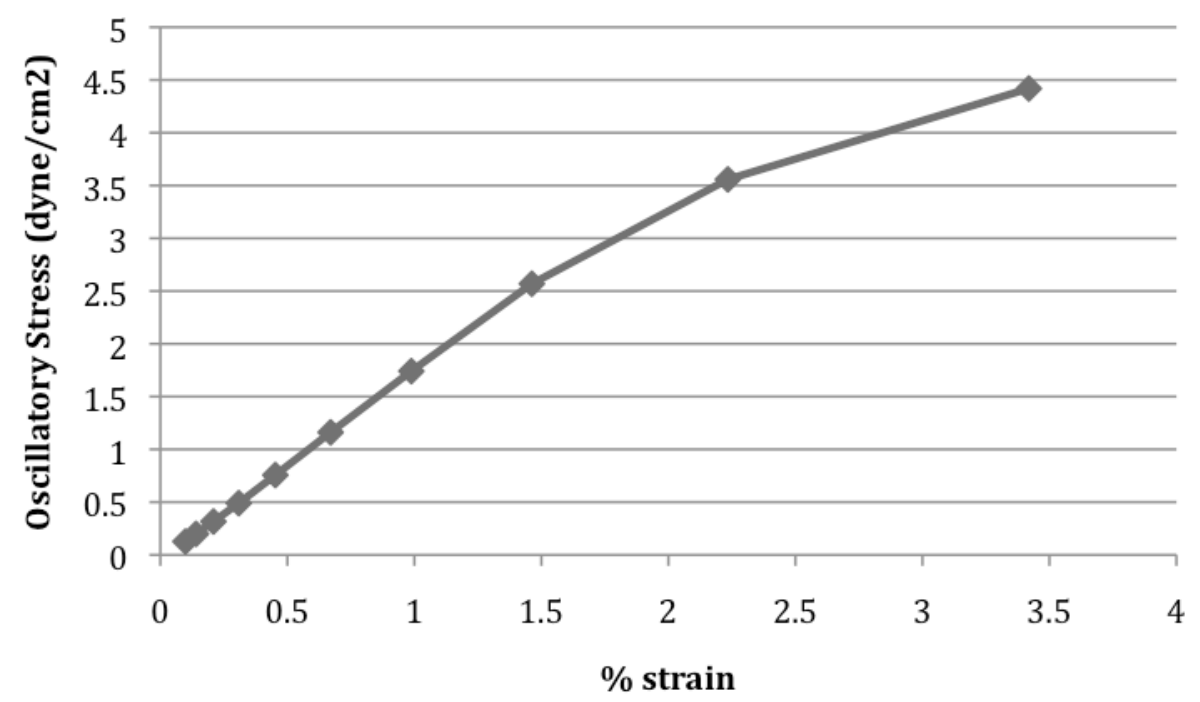

Figure 4-19: Stress-strain curve for PR_g to determine linear viscoelastic regime

A $12 \mathrm{mM}$ solution of PR_g was prepared and immediately pipetted onto a Peltier plate maintained at a temperature of $25^{\circ} \mathrm{C}$. A time sweep was performed using a frequency of $1 \mathrm{rad} / \mathrm{s}$ and a strain of $0.5 \%$ (Figure 4-20). At the gelation point the viscous modulus, $G^{\prime \prime}$ is becomes less than the elastic modulus, $G^{\prime}$, and this crossover is the defining characteristic between a gel and a viscous liquid. The time sweep indicates that gelation time of a $12 \mathrm{mM}$ solution of PR_g amphiphile is approximately $30 \mathrm{~min}$. The moduli continued to increase over the course of the measurement, indicating that the gel was still forming cross-links. After approximately 2 days the moduli remained fairly constant. 


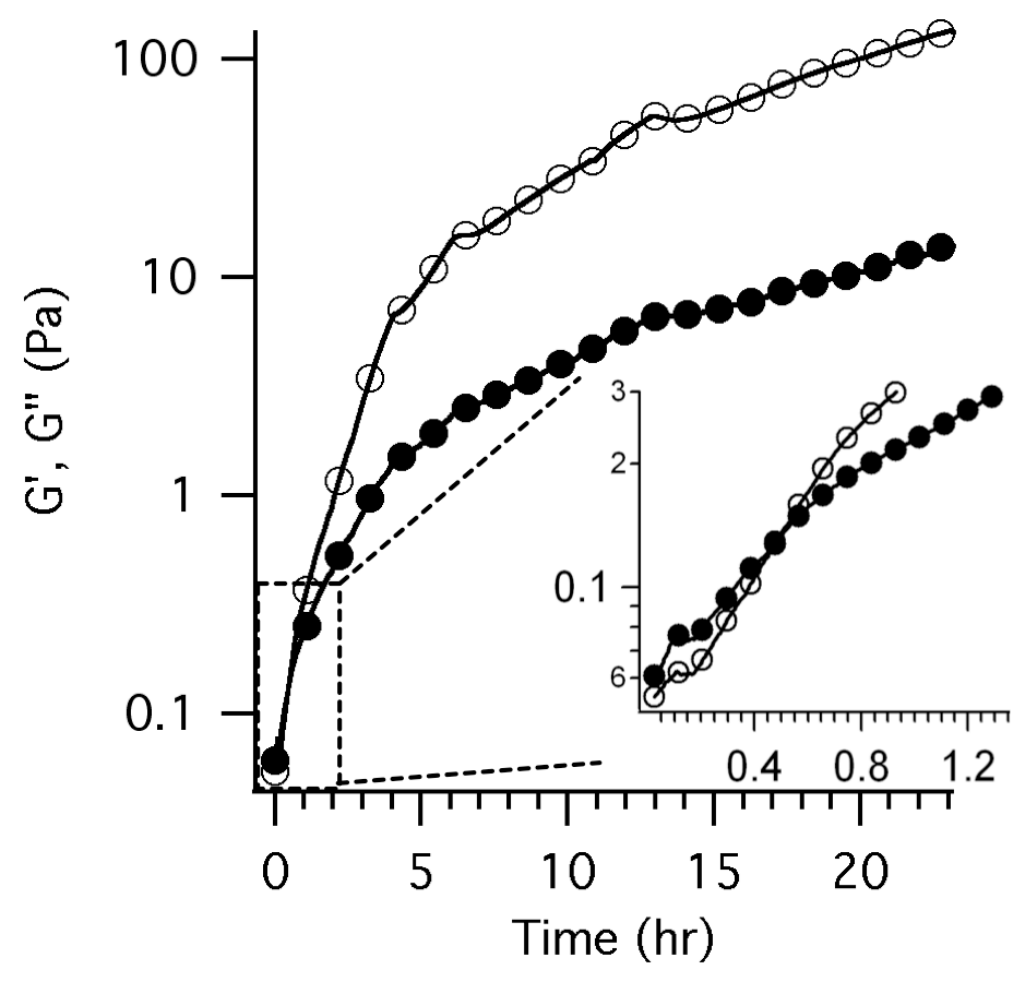

Figure 4-20: PR_g gelation of a $12 \mathrm{mM}$ sample in Milli-Q water monitored by oscillating plate rheometry at a frequency of $1 \mathrm{rad} / \mathrm{s}$. Elastic modulus, $G^{\prime}(0)$, and viscous modulus, $G^{\prime \prime}(\bullet)$, of a PR_g solution measured as a function of time. Gelation point (signified by $G^{\prime}>G^{\prime \prime}$ ) is observed at $30 \mathrm{~min}$. The inset shows the crossover between $G^{\prime}$ and $G^{\prime \prime}$ in greater detail. For clarity only every $300^{\text {th }}$ data point in the larger graph and every $20^{\text {th }}$ data point in the inset were plotted. The results are representative of $n=3$ (three independent experiments performed on different days), but data are shown from a single experiment.

Another important characteristic of gels with a high degree of cross-linking is that $G^{\prime}$ and $G^{\prime \prime}$ are minimally sensitive to the oscillation frequency. To test the degree of PR_g cross-links in the gel, a $10 \mathrm{mM}$ solution of PR_g amphiphile was allowed to age in a sealed chamber for $48 \mathrm{hrs}$ at room temperature. The sealed chamber was constructed by adhering a silicone O-ring to a glass coverslip using silicone rubber and covering to the top of the chamber with a glass slide lightly coated with vacuum grease. The glass coverslip was adhered to a Peltier plate with vacuum grease, and the Peltier plate kept the sample at 
$25^{\circ} \mathrm{C}$ for the duration of the rheology measurement. A frequency sweep from 0.01 to $100 \mathrm{rad} / \mathrm{s}$ was performed on the gel using a $13.07 \mathrm{~mm}$ parallel plate oscillated at $1 \%$ strain.

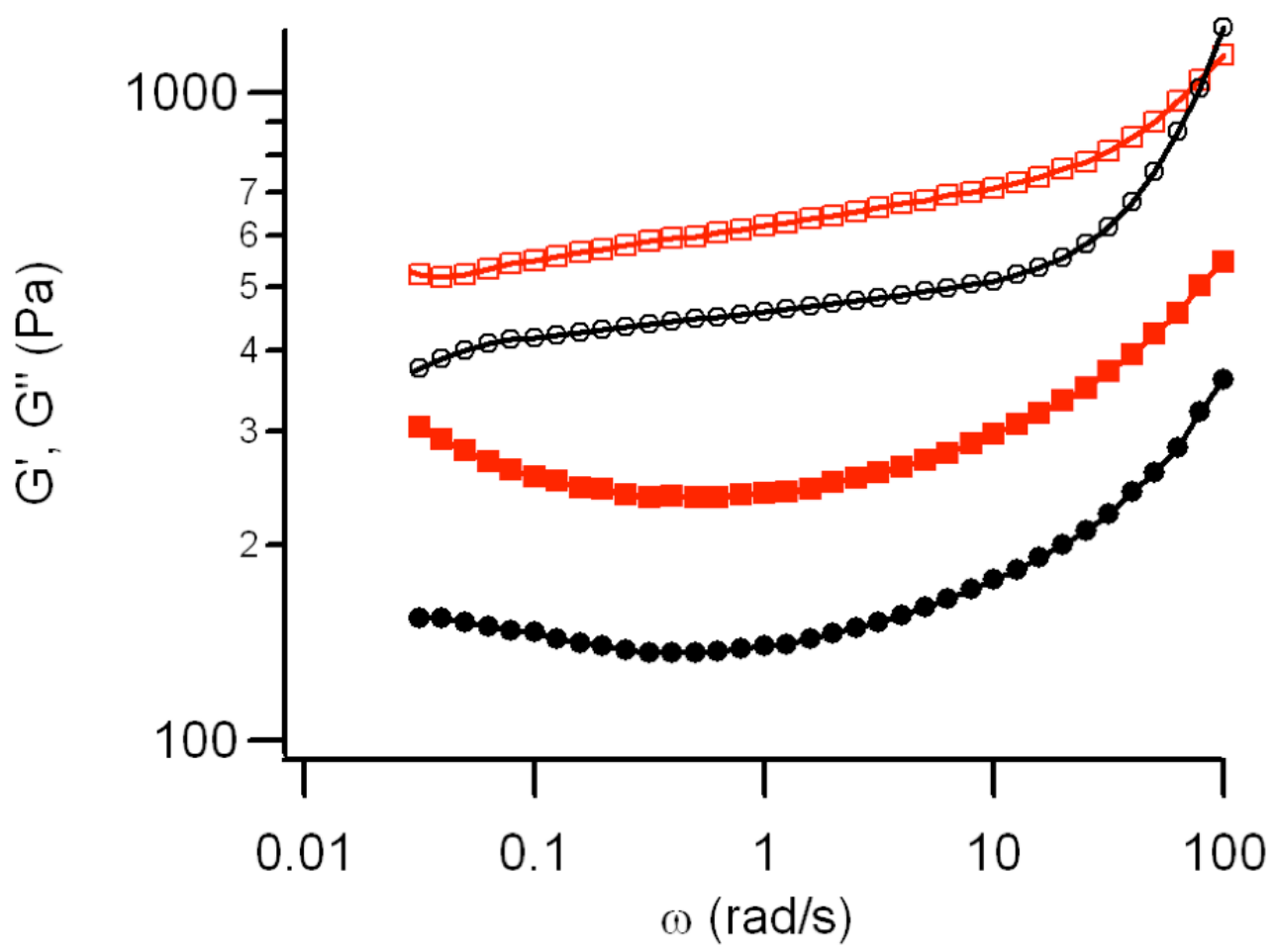

Figure 4-21: Frequency sweep showing $G^{\prime}$ and $G^{\prime \prime}$ of a PR_g gel. A 10 mM PR_g gel was prepared and aged 48 hours (circles) or for 1 month (squares) in a sealed chamber before rheometry measurement. Both $G^{\prime}$ (open symbols) and $G^{\prime \prime}$ (closed symbols) were not significantly affected by changes in oscillation frequency. The results are representative of $n=2$ (two independent experiments performed on different days), but data are shown from a single experiment.

The data show that $G^{\prime}$ and $G^{\prime \prime}$ are minimally affected by the frequency change between 0.1 and $10 \mathrm{rad} / \mathrm{s}$, and the moduli do not cross in the frequency range tested (Figure 4-21). This indicates that the PR_g nanofibers are forming crosslinks between nanofibers, and the gel behavior is not merely due to entangled nanofibers. In the case of PR_g, these cross-links are most likely noncovalent bonds, such as hydrogen bonds. To see if the gel would continue to stiffen over time, a 10 mM PR_g amphiphile gel was allowed to age in a sealed chamber for 
1 month, and then another frequency sweep was taken. The gel showed a slight increase in both $G^{\prime}$ and $G^{\prime \prime}$. Over the course of 1 month, some water evaporation is expected, but the sealed chamber prevented most of the evaporation.

An important consideration in tissue design is mimicking the mechanical properties of native tissues. Neural tissue has a modulus of approximately $E \approx$ $0.1 \mathrm{kPa}$ and muscle tissue is $E \approx 10 \mathrm{kPa}$ where $E$ is the Young's modulus. ${ }^{68}$ The relationship between the shear modulus, $G$, and $E$ is described by Equation 4-8, where $v$ is Poisson's ratio. ${ }^{117}$

Equation 4-8 ${ }^{117}$

$E=2 G(1+v)$

Poisson's ratio describes the change in volume, $V$, of a material when it is deformed by an extensional strain, $\varepsilon$ (Equation 4-9). ${ }^{117}$ As a material is stretched in one direction, it tends to contract in the directions perpendicular to direction of the force.

\section{Equation 4-9 ${ }^{117}$}

$v=\frac{1}{2}\left(1-\frac{1}{V} \frac{d V}{d \varepsilon}\right)$

For most soft materials, the volume remains constant especially under low deformation, so $v=0.5$. This reduces Equation $4-8$ to $E \approx 3 G$. The shear modulus, $G$, is calculated by:

$$
\begin{aligned}
& \text { Equation 4-10 } \\
& G=\sqrt{\left(G^{\prime}\right)^{2}+\left(G^{\prime \prime}\right)^{2}}
\end{aligned}
$$


At a frequency of $1 \mathrm{rad} / \mathrm{s}$, the $G$ of $P R \_g$ is $480 \mathrm{~Pa}$. Thus the modulus of elasticity $(E)$ is approximately $1.5 \mathrm{kPa}$, which is within the range of modulus of elasticity for living tissues. 


\section{Chapter 5 Conclusion}

Tissue engineering promises to completely change the treatment of disease. One of the most important components of an engineered tissue is the scaffold, which provides structure to the tissue and a location for cells to adhere. This work focused on improving cell adhesion to scaffolds for tissue engineering by designing a biomimetic peptide targeting the $\alpha_{5} \beta_{1}$ integrin.

Six fibronectin mimetic peptide-amphiphiles with dialkyl tails were designed incorporating the primary binding domain, RGD, and synergy-binding domain, PHSRN, found in the $a_{5} \beta_{1}$ integrin binding site in fibronectin. The peptide amphiphiles varied the linker that connected the two binding domains and the spacer separating the tail from the binding domains in the peptide headgroup. The original hypothesis in the Kokkoli group was that both the linker length and hydrophilicity/hydrophobicity of the amino acids in the linker were important for $a_{5} \beta_{1}$ integrin binding.

The peptide-amphiphile surfaces were fabricated using the Langmuir-Blodgett technique. LB isotherms showed that sequences containing high levels of serine packed more tightly than the sequences containing fewer serines. PR_d contained the most serines and did not transfer well to the mica substrates. AFM images of the LB membranes displayed shallow divots, which may indicate that the peptide-amphiphile headgroups are bent at the interface, exposing the PHSRN domain. The divots on the PR_d surface showed two depths, which may be an indication of missing peptide-amphiphiles in the membrane. Parallel AFM and cell studies undertaken by other group members showed that PR_b bound an $\alpha_{5} \beta_{1}$ integrin-functionalized AFM tip better than the other studied sequences and that PR_b surfaces also adhered HUVEC cells 
more strongly than other surfaces. These results support the hypothesis that the hydrophilicity/hydrophobicity of the serine/glycine linker of PR_b is a better linker for cell attachment.

A fibronectin mimetic peptide, PR_g (a modified version of $P R \_b$ ), was designed to contain both the primary and synergy-binding domains of fibronectin. PR_g was coupled to a single hydrocarbon tail to make a peptideamphiphile to self-assemble into nanofibers in solution. PR_g was found to form a gel after the addition of Milli-Q water to the lyophilized peptide-amphiphile. PR_g did not require the screening of its charge before self-assembly and gelation, which is a significant difference from other peptide-amphiphiles reported in the literature that formed nanofibers and gelled. The critical micelle concentration of the nanofibers was measured by lipophilic-staining to be $38 \mu \mathrm{M}$. Cryo-TEM images of the peptide-amphiphile nanofibers showed single nanofibers with a diameter of approximately $10 \mathrm{~nm}$, and at higher concentrations, the nanofibers bundle together. The entanglements that the bundles formed could give rise to the gelation seen at higher concentrations. SANS experiments agreed with the cryo-TEM images and showed that the peptide-amphiphile forms a rod-like structure in solution. CD spectroscopy indicated that the PR_g peptide showed a random secondary structure; whereas, after an aging period, PR_g peptide-amphiphile's headgroup showed an $\alpha+\beta$ secondary structure. Rheological measurements of PR_g gels show that the elastic modulus approaches the elasticity of tissues found in vivo, which is important for future consideration of the nanofiber gel as a tissue engineering scaffold. 


\section{References}

1. UNOS United Network for Organ Sharing. http://www.unos.org (1/15/10)

2. Langer, R.; Vacanti, J. P., Tissue Engineering. Science 1993, 260, (5110), 920-926.

3. Alberts, B.; Johnson, A.; Lewis, J.; Raff, M.; Roberts, K.; Walter, P., Molecular Biology of the Cell. 4th ed.; Garland Science: New York, 2002.

4. Leahy, D. J.; Aukhil, I.; Erickson, H. P., 2.0 angstrom crystal structure of a four-domain segment of human fibronectin encompassing the RGD loop and synergy region. Cell 1996, 84, (1), 155-164.

5. $\quad$ Petersen, T. E.; Thogersen, H. C.; Skorstengaard, K.; Vibepedersen, K.; Sahl, P.; Sottrupjensen, L.; Magnusson, S., Partial primary structure of bovine plasma fibronectin - 3 types of internal homology. Proceedings of the National Academy of Sciences of the United States of AmericaBiological Sciences 1983, 80, (1), 137-141.

6. Kornblihtt, A. R.; Umezawa, K.; Vibepedersen, K.; Baralle, F. E., Primary structure of human fibronectin - differential splicing may generate at least 10 polypeptides from a single gene. Embo Journal 1985, 4, (7), 17551759.

7. Hautanen, A.; Gailit, J.; Mann, D. M.; Ruoslahti, E., Effects of modifications of the RGD sequence and its context on recognition by the fibronectin receptor. Journal of Biological Chemistry 1989, 264, (3), 1437-1442.

8. Mould, A. P.; Askari, J. A.; Aota, S.; Yamada, K. M.; Irie, A.; Takada, Y.; Mardon, H. J.; Humphries, M. J., Defining the topology of integrin alpha 5 beta 1-fibronectin interactions using inhibitory anti-alpha 5 and anti-beta 1 monoclonal antibodies - Evidence that the synergy sequence of fibronectin is recognized by the amino-terminal repeats of the alpha 5 subunit. Journal of Biological Chemistry 1997, 272, (28), 17283-17292.

9. Hynes, R. O., Integrins: Bidirectional, allosteric signaling machines. Cell 2002, 110, (6), 673-687.

10. Humphries, J. D.; Askari, J. A.; Zhang, X. P.; Takada, Y.; Humphries, M. J.; Mould, A. P., Molecular basis of ligand recognition by integrin alpha(5)beta(1) - II. Specificity of Arg-Gly-Asp binding is determined by 
Trp(157) of the alpha subunit. Journal of Biological Chemistry 2000, 275, (27), 20337-20345.

11. Mould, A. P.; Akiyama, S. K.; Humphries, M. J., Regulation of integrin alpa-5-beta-1-fibronectin interactions by divalent-cations - evidence for distinct classes of binding-sites for $\mathrm{Mn2}+, \mathrm{Mg} 2+$, and $\mathrm{Ca} 2+$. Journal of Biological Chemistry 1995, 270, (44), 26270-26277.

12. Qu, A. D.; Leahy, D. J., The role of the divalent cation in the structure of the I domain from the CD11a/CD18 integrin. Structure 1996, 4, (8), 931942.

13. Livant, D. L.; Brabec, R. K.; Kurachi, K.; Allen, D. L.; Wu, Y. L.; Haaseth, R.; Andrews, P.; Ethier, S. P.; Markwart, S., The PHSRN sequence induces extracellular matrix invasion and accelerates wound healing in obese diabetic mice. Journal of Clinical Investigation 2000, 105, (11), 1537-1545.

14. Stupack, D. G.; Cheresh, D. A., Integrins and angiogenesis. In Current Topics in Developmental Biology, Vol 64, 2004; Vol. 64, pp 207-238.

15. Mettouchi, A.; Meneguzzi, G., Distinct roles of beta 1 integrins during angiogenesis. European Journal of Cell Biology 2006, 85, (3-4), 243-247.

16. Milner, R.; Campbell, I. L., The integrin family of cell adhesion molecules has multiple functions within the CNS. Journal of Neuroscience Research 2002, 69, (3), 286-291.

17. Taverna, D.; Hynes, R. O., Reduced blood vessel formation and tumor growth in alpha 5-integrin-negative teratocarcinomas and embryoid bodies. Cancer Research 2001, 61, (13), 5255-5261.

18. Kim, S.; Bell, K.; Mousa, S. A.; Varner, J. A., Regulation of angiogenesis in vivo by ligation of integrin alpha 5 beta 1 with the central cell-binding domain of fibronectin. American Journal of Pathology 2000, 156, (4), 1345-1362.

19. Francis, S. E.; Goh, K. L.; Hodivala-Dilke, K.; Bader, B. L.; Stark, M.; Davidson, D.; Hynes, R. O., Central roles of alpha(5)beta(1) integrin and fibronectin in vascular development in mouse embryos and embryoid bodies. Arteriosclerosis Thrombosis and Vascular Biology 2002, 22, (6), 927-933. 
20. Mettouchi, A.; Klein, S.; Guo, W. J.; Lopez-Lago, M.; Lemichez, E.; Westwick, J. K.; Giancotti, F. G., Integrin-specific activation of Rac controls progression through the $\mathrm{G}(1)$ phase of the cell cycle. Molecular Cell 2001, 8, (1), 115-127.

21. Byzova, T. V.; Goldman, C. K.; Pampori, N.; Thomas, K. A.; Bett, A.; Shattil, S. J.; Plow, E. F., A mechanism for modulation of cellular responses to VEGF: Activation of the integrins. Molecular Cell 2000, 6, (4), 851-860.

22. Bayless, K. J.; Salazar, R.; Davis, G. E., RGD-dependent vacuolation and lumen formation observed during endothelial cell morphogenesis in three-dimensional fibrin matrices involves the alpha(v)beta(3) and alpha(5)beta(1) integrins. American Journal of Pathology 2000, 156, (5), 1673-1683.

23. Serini, G.; Valdembri, D.; Zanivan, S.; Morterra, G.; Burkhardt, C.; Caccavari, F.; Zammataro, L.; Primo, L.; Tamagnone, L.; Logan, M.; Tesssier-Lavigne, M.; Taniguchi, M.; Puschel, A. W.; Bussolino, F., Class 3 semaphorins control vascular morphogenesis by inhibiting integrin function. Nature 2003, 424, (6947), 391-397.

24. Discher, D. E.; Janmey, P.; Wang, Y. L., Tissue cells feel and respond to the stiffness of their substrate. Science 2005, 310, (5751), 1139-1143.

25. Ruoslahti, E.; Pierschbacher, M. D., New perspectives in cell-adhesion RGD and integrins. Science 1987, 238, (4826), 491-497.

26. Palsson, B.; Bhatia, S., Tissue engineering. Pearson Prentice Hall: Upper Saddle River, N.J., 2004; p xviii, 407 p.

27. Lewandowska, K.; Pergament, E.; Sukenik, C. N.; Culp, L. A., Cell-typespecific adhesion mechanisms mediated by fibronectin adsorbed to chemically derivatized substrata. Journal of Biomedical Materials Research 1992, 26, (10), 1343-1363.

28. Iuliano, D. J.; Saavedra, S. S.; Truskey, G. A., Effect of the conformation and orientation of adsorbed fibronectin on endothelial-cell spreading and the strength of adhesion. Journal of Biomedical Materials Research 1993, 27, (8), 1103-1113.

29. Hlady, V.; Buijs, J., Protein adsorption on solid surfaces. Current Opinion in Biotechnology 1996, 7, (1), 72-77. 
30. Lhoest, J. B.; Detrait, E.; van den Bosch de Aguilar, P.; Bertrand, P., Fibronectin adsorption, conformation, and orientation on polystyrene substrates studied by radiolabeling, XPS, and ToF SIMS. Journal of Biomedical Materials Research 1998, 41, (1), 95-103.

31. Williams, S. K.; Jarrell, B. E.; Friend, L.; Radomski, J. S.; Carabasi, R. A.; Koolpe, E.; Mueller, S. N.; Thornton, S. C.; Marinucci, T.; Levine, E., Adult human-endothelial cell compatibility with prosthetic graft material. Journal of Surgical Research 1985, 38, (6), 618-629.

32. Seeger, J. M.; Klingman, N., Improved invivo endothelialization of prosthetic grafts by surface modification with fibronectin. Journal of Vascular Surgery 1988, 8, (4), 476-482.

33. Kaehler, J.; Zilla, P.; Fasol, R.; Deutsch, M.; Kadletz, M., Precoating substrate and surface configuration determine adherence and spreading of seeded endothelial-cells on polytetrafluoroethylene grafts. Journal of Vascular Surgery 1989, 9, (4), 535-541.

34. Kobayashi, H.; Ikada, Y., Corneal cell-adhesion and proliferation on hydrogel sheets bound with cell-adhesive proteins. Current Eye Research 1991, 10, (10), 899-908.

35. Tinois, E.; Tiollier, J.; Gaucherand, M.; Dumas, H.; Tardy, M.; Thivolet, J., Invitro and posttransplantation differentiation of human keratinocytes grown on the human type-IV collagen film of a bilayered dermal substitute. Experimental Cell Research 1991, 193, (2), 310-319.

36. Mooney, D.; Hansen, L.; Vacanti, J.; Langer, R.; Farmer, S.; Ingber, D., Switching from differentiation to growth in hepatocytes control by extracellular-matrix. Journal of Cellular Physiology 1992, 151, (3), 497505.

37. Ghosh, K.; Ren, X. D.; Shu, X. Z.; Prestwich, G. D.; Clark, R. A. F., Fibronectin functional domains coupled to hyaluronan stimulate adult human dermal fibroblast responses critical for wound healing. Tissue Engineering 2006, 12, (3), 601-613.

38. Elbert, D. L.; Hubbell, J. A., Surface treatments of polymers for biocompatibility. Annual Review of Materials Science 1996, 26, 365-394.

39. Mosahebi, A.; Wiberg, M.; Terenghi, G., Addition of fibronectin to alginate matrix improves peripheral nerve regeneration in tissue-engineered conduits. Tissue Engineering 2003, 9, (2), 209-218. 
40. Petsko, G. A.; Ringe, D., Protein structure and function. New Science; Sinauer Associates; Blackwell: London Sunderland, Mass. Oxford, UK, 2004; $p$ xxii, 195 p.

41. Glass, J. R.; Dickerson, K. T.; Stecker, K.; Polarek, J. W., Characterization of a hyaluronic acid-Arg-Gly-Asp peptide cell attachment matrix. Biomaterials 1996, 17, (11), 1101-1108.

42. Hern, D. L.; Hubbell, J. A., Incorporation of adhesion peptides into nonadhesive hydrogels useful for tissue resurfacing. Journal of Biomedical Materials Research 1998, 39, (2), 266-276.

43. Griffith, L. G.; Lopina, S., Microdistribution of substratum-bound ligands affects cell function: hepatocyte spreading on PEO-tethered galactose. Biomaterials 1998, 19, (11-12), 979-986.

44. Barrera, D. A.; Zylstra, E.; Lansbury, P. T.; Langer, R., Synthesis and RGD peptide modification of a new biodegradable copollymer poly(lactic acid-co-lysine). Journal of the American Chemical Society 1993, 115, (23), 11010-11011.

45. Kugo, K.; Okuno, M.; Masuda, K.; Nishino, J.; Masuda, H.; Iwatsuki, M., Fibroblast attachment to Arg-Gly-Asp peptide-immobilized poly(gammamethyl L-glutamate). Journal of Biomaterials Science-Polymer Edition 1994, 5, (4), 325-337.

46. Cook, A. D.; Hrkach, J. S.; Gao, N. N.; Johnson, I. M.; Pajvani, U. B.; Cannizzaro, S. M.; Langer, R., Characterization and development of RGD-peptide-modified poly(lactic acid-co-lysine) as an interactive, resorbable biomaterial. Journal of Biomedical Materials Research 1997, 35, (4), 513-523.

47. Rezania, A.; Thomas, C. H.; Branger, A. B.; Waters, C. M.; Healy, K. E., The detachment strength and morphology of bone cells contacting materials modified with a peptide sequence found within bone sialoprotein. Journal of Biomedical Materials Research 1997, 37, (1), 919.

48. Porte-Durrieu, M. C.; Labrugere, C.; Villars, F.; Lefebvre, F.; Dutoya, S.; Guette, A.; Bordenave, L.; Baquey, C., Development of RGD peptides grafted onto silica surfaces: XPS characterization and human endothelial cell interactions. Journal of Biomedical Materials Research 1999, 46, (3), 368-375. 
49. Roberts, C.; Chen, C. S.; Mrksich, M.; Martichonok, V.; Ingber, D. E.; Whitesides, G. M., Using mixed self-assembled monolayers presenting $\mathrm{RGD}$ and $(\mathrm{EG})(3) \mathrm{OH}$ groups to characterize long-term attachment of bovine capillary endothelial cells to surfaces. Journal of the American Chemical Society 1998, 120, (26), 6548-6555.

50. Lahiri, J.; Ostuni, E.; Whitesides, G. M., Patterning ligands on reactive SAMs by microcontact printing. Langmuir 1999, 15, (6), 2055-2060.

51. Massia, S. P.; Hubbell, J. A., Vascular endothelial-cell adhesion and spreading promoted by the peptide REDV of the IIICS region of plasma fibronectin is mediated by integrin alpha-4-beta-1. Journal of Biological Chemistry 1992, 267, (20), 14019-14026.

52. Pierschbacher, M. D.; Ruoslahti, E., Cell attachment activity of fibronectin can be duplicated by small synthetic fragments of the molecule. Nature 1984, 309, (5963), 30-33.

53. Ruoslahti, E., RGD and other recognition sequences for integrins. Annual Review of Cell and Developmental Biology 1996, 12, 697-715.

54. Graf, J.; Iwamoto, Y.; Sasaki, M.; Martin, G. R.; Kleinman, H. K.; Robey, F. A.; Yamada, Y., Identification of an amino-acid-sequence in laminin mediating cell attachment, chemotaxis, and receptor-binding. Cell 1987, 48, (6), 989-996.

55. Tashiro, K.; Sephel, G. C.; Weeks, B.; Sasaki, M.; Martin, G. R.; Kleinman, H. K.; Yamada, Y., A synthetic peptide containing the IKVAV sequence from the a-chain of laminin mediates cell attachment, migration, and neurite outgrowth. Journal of Biological Chemistry 1989, 264, (27), 16174-16182.

56. Massia, S. P.; Rao, S. S.; Hubbell, J. A., Covalently immobilized laminin peptide Tyr-lle-Gly-Ser-Arg (YIGSR) supports cell spreading and colocalization of the 67-kilodalton laminin receptor with alpha-actinin and vinculin. Journal of Biological Chemistry 1993, 268, (11), 8053-8059.

57. Staatz, W. D.; Fok, K. F.; Zutter, M. M.; Adams, S. P.; Rodriguez, B. A.; Santoro, S. A., Identification of a tetrapeptide recognition sequence for the alpha-2-beta-1-integrin in collagen. Journal of Biological Chemistry 1991, 266, (12), 7363-7367.

58. Underwood, P. A.; Bennett, F. A.; Kirkpatrick, A.; Bean, P. A.; Moss, B. A., Evidence for the location of a binding sequence for the alpha-2-beta-1 
integrin of endothelial-cells, in the beta-1 subunit of laminin. Biochemical Journal 1995, 309, 765-771.

59. Pocza, P.; Suli-Vargha, H.; Darvas, Z.; Falus, A., Locally generated VGVAPG and VAPG elastin-derived peptides amplify melanoma invasion via the galectin-3 receptor. International Journal of Cancer 2008, 122, (9), 1972-1980.

60. Kloczewiak, M.; Timmons, S.; Lukas, T. J.; Hawiger, J., Platelet receptor recognition site on human-fibrinogen - synthesis and structure-function relationship of peptides corresponding to the carboxy-terminal segment of the gamma-chain. Biochemistry 1984, 23, (8), 1767-1774.

61. Altieri, D. C.; Plescia, J.; Plow, E. F., The structural motif glycine-190valine-202 of the fibrinogen-gamma chain interacts with CD11B CD18 integrin (alpha-M-beta-2, MAC-1) and promotes leukocyte adhesion. Journal of Biological Chemistry 1993, 268, (3), 1847-1853.

62. Doroski, D. M.; Brink, K. S.; Temenoff, J. S., Techniques for biological characterization of tissue-engineered tendon and ligament. Biomaterials 2007, 28, (2), 187-202.

63. Riveline, D.; Zamir, E.; Balaban, N. Q.; Schwarz, U. S.; Ishizaki, T.; Narumiya, S.; Kam, Z.; Geiger, B.; Bershadsky, A. D., Focal contacts as mechanosensors: Externally applied local mechanical force induces growth of focal contacts by an mDia1-dependent and ROCK-independent mechanism. Journal of Cell Biology 2001, 153, (6), 1175-1185.

64. Bershadsky, A. D.; Balaban, N. Q.; Geiger, B., Adhesion-dependent cell mechanosensitivity. Annual Review of Cell and Developmental Biology 2003, 19, 677-695.

65. Balaban, N. Q.; Schwarz, U. S.; Riveline, D.; Goichberg, P.; Tzur, G.; Sabanay, I.; Mahalu, D.; Safran, S.; Bershadsky, A.; Addadi, L.; Geiger, B., Force and focal adhesion assembly: a close relationship studied using elastic micropatterned substrates. Nature Cell Biology 2001, 3, (5), 466-472.

66. Lo, C. M.; Wang, H. B.; Dembo, M.; Wang, Y. L., Cell movement is guided by the rigidity of the substrate. Biophysical Journal 2000, 79, (1), 144-152.

67. Davies, P. F.; Barbee, K. A.; Volin, M. V.; Robotewskyj, A.; Chen, J.; Joseph, L.; Griem, M. L.; Wernick, M. N.; Jacobs, E.; Polacek, D. C.; 
DePaola, N.; Barakat, A. I., Spatial relationships in early signaling events of flow-mediated endothelial mechanotransduction. Annual Review of Physiology 1997, 59, 527-549.

68. Engler, A. J.; Griffin, M. A.; Sen, S.; Bonnetnann, C. G.; Sweeney, H. L.; Discher, D. E., Myotubes differentiate optimally on substrates with tissuelike stiffness: pathological implications for soft or stiff microenvironments. Journal of Cell Biology 2004, 166, (6), 877-887.

69. Gray, D. S.; Tien, J.; Chen, C. S., Repositioning of cells by mechanotaxis on surfaces with micropatterned Young's modulus. Journal of Biomedical Materials Research Part A 2003, 66A, (3), 605-614.

70. Brandl, F.; Sommer, F.; Goepferich, A., Rational design of hydrogels for tissue engineering: Impact of physical factors on cell behavior. Biomaterials 2007, 28, (2), 134-146.

71. Mahoney, M. J.; Anseth, K. S., Three-dimensional growth and function of neural tissue in degradable polyethylene glycol hydrogels. Biomaterials 2006, 27, (10), 2265-2274.

72. Shen, J. Y.; Pan, X. Y.; Lim, C. H.; Chan-Park, M. B.; Zhu, X.; Beuerman, R. W., Synthesis, characterization, and in vitro degradation of a biodegradable photo-cross-linked film from liquid poly(epsiloncaprolactone-co-lactide-co-glycolide) diacrylate. Biomacromolecules 2007, 8, (2), 376-385.

73. Folkman, J.; Hochberg, M., Self-regulation of growth in three dimensions. The Journal of Experimental Medicine 1973, 138, (4), 745-753.

74. Colton, C. K., Implantable biohybrid artificial organs. Cell Transplantation 1995, 4, (4), 415-436.

75. Laschke, M. W.; Harder, Y.; Amon, M.; Martin, I.; Farhadi, J.; Ring, A.; Torio-Padron, N.; Schramm, R.; Rucker, M.; Junker, D.; Haufel, J. M.; Carvalho, C.; Heberer, M.; Germann, G.; Vollmar, B.; Menger, M. D., Angiogenesis in tissue engineering: Breathing life into constructed tissue substitutes. Tissue Engineering 2006, 12, (8), 2093-2104.

76. Zisch, A. H.; Lutolf, M. P.; Ehrbar, M.; Raeber, G. P.; Rizzi, S. C.; Davies, N.; Schmokel, H.; Bezuidenhout, D.; Djonov, V.; Zilla, P.; Hubbell, J. A., Cell-demanded release of VEGF from synthetic, biointeractive cellingrowth matrices for vascularized tissue growth. Faseb Journal 2003, $17,(13), 2260-+$. 
77. Israelachvili, J. N., Inermolecular and Surfaces Forces: with Applications to Colloidal and Biological Systems. Academic Press: London, 1985.

78. Lowik, D.; Garcia-Hartjes, J.; Meijer, J. T.; van Hest, J. C. M., Tuning secondary structure and self-assembly of amphiphilic peptides. Langmuir 2005, 21, (2), 524-526.

79. Gore, T.; Dori, Y.; Talmon, Y.; Tirrell, M.; Bianco-Peled, H., Selfassembly of model collagen peptide amphiphiles. Langmuir 2001, 17, (17), 5352-5360.

80. Greenfield, M. A.; Palmer, L. C.; Vernizzi, G.; de la Cruz, M. O.; Stupp, S. I., Buckled membranes in mixed-valence ionic amphiphile vesicles. Journal of the American Chemical Society 2009, 131, (34), 12030-+.

81. Hartgerink, J. D.; Beniash, E.; Stupp, S. I., Self-assembly and mineralization of peptide-amphiphile nanofibers. Science 2001, 294, (5547), 1684-1688.

82. Berndt, P.; Fields, G. B.; Tirrell, M., Synthetic lipidation of peptides and amino-acids - monolayer structure and properties. Journal of the American Chemical Society 1995, 117, (37), 9515-9522.

83. Vauthey, S.; Santoso, S.; Gong, H. Y.; Watson, N.; Zhang, S. G., Molecular self-assembly of surfactant-like peptides to form nanotubes and nanovesicles. Proceedings of the National Academy of Sciences of the United States of America 2002, 99, (8), 5355-5360.

84. Cui, H.; Muraoka, T.; Cheetham, A. G.; Stupp, S. I., Self-assembly of giant peptide nanobelts. Nano Letters 2009, 9, (3), 945-951.

85. Zana, R., Micelles and Vesicles. Encyclopedia of Supramolecular Chemistry 2004, 861 - 867.

86. Dori, Y.; Bianco-Peled, H.; Satija, S. K.; Fields, G. B.; McCarthy, J. B.; Tirrell, M., Ligand accessibility as means to control cell response to bioactive bilayer membranes. Journal of Biomedical Materials Research 2000, 50, (1), 75-81.

87. Fields, G. B.; Lauer, J. L.; Dori, Y.; Forns, P.; Yu, Y. C.; Tirrell, M., Proteinlike molecular architecture: Biomaterial applications for inducing cellular receptor binding and signal transduction. Biopolymers 1998, 47, (2), 143-151. 
88. Yu, Y. C.; Pakalns, T.; Dori, Y.; McCarthy, J. B.; Tirrell, M.; Fields, G. B., Construction of biologically active protein molecular architecture using self-assembling peptide-amphiphiles. In Solid-Phase Peptide Synthesis, 1997; Vol. 289, pp 571-587.

89. Massia, S. P.; Hubbell, J. A., Immobilized amines and basic-amino-acids as mimetic heparin-binding domains for cell-surface proteoglycanmediated adhesion. Journal of Biological Chemistry 1992, 267, (14), 10133-10141.

90. Capito, R. M.; Azevedo, H. S.; Velichko, Y. S.; Mata, A.; Stupp, S. I., Selfassembly of large and small molecules into hierarchically ordered sacs and membranes. Science 2008, 319, (5871), 1812-1816.

91. Garg, A.; Tisdale, A. W.; Haidari, E.; Kokkoli, E., Targeting colon cancer cells using PEGylated liposomes modified with a fibronectin-mimetic peptide. International Journal of Pharmaceutics 2009, 366, (1-2), 201210.

92. Tirrell, M.; Kokkoli, E.; Biesalski, M., The role of surface science in bioengineered materials. Surface Science 2002, 500, (1-3), 61-83.

93. Park, T. H.; Shuler, M. L., Integration of cell culture and microfabrication technology. Biotechnology Progress 2003, 19, (2), 243-253.

94. Erickson, D.; Li, D. Q.; Krull, U. J., Modeling of DNA hybridization kinetics for spatially resolved biochips. Analytical Biochemistry 2003, 317, (2), 186-200.

95. Yamato, M.; Konno, C.; Utsumi, M.; Kikuchi, A.; Okano, T., Thermally responsive polymer-grafted surfaces facilitate patterned cell seeding and co-culture. Biomaterials 2002, 23, (2), 561-567.

96. Malkar, N. B.; Lauer-Fields, J. L.; Juska, D.; Fields, G. B., Characterization of peptide-amphiphiles possessing cellular activation sequences. Biomacromolecules 2003, 4, (3), 518-528.

97. Dillow, A. K.; Ochsenhirt, S. E.; McCarthy, J. B.; Fields, G. B.; Tirrell, M., Adhesion of alpha(5)beta(1) receptors to biomimetic substrates constructed from peptide amphiphiles. Biomaterials 2001, 22, (12), 14931505. 
98. Kokkoli, E.; Ochsenhirt, S. E.; Tirrell, M., Collective and single-molecule interactions of alpha(5)beta(1) integrins. Langmuir 2004, 20, (6), 23972404.

99. Mardilovich, A.; Kokkoli, E., Biomimetic peptide-amphiphiles for functional biomaterials: The role of GRGDSP and PHSRN. Biomacromolecules 2004, 5, (3), 950-957.

100. Arnold, M. S.; Guler, M. O.; Hersam, M. C.; Stupp, S. I., Encapsulation of carbon nanotubes by self-assembling peptide amphiphiles. Langmuir 2005, 21, (10), 4705-4709.

101. Hartgerink, J. D.; Beniash, E.; Stupp, S. I., Peptide-amphiphile nanofibers: A versatile scaffold for the preparation of self-assembling materials. Proceedings of the National Academy of Sciences of the United States of America 2002, 99, (8), 5133-5138.

102. Beniash, E.; Hartgerink, J. D.; Storrie, H.; Stendahl, J. C.; Stupp, S. I., Self-assembling peptide amphiphile nanofiber matrices for cell entrapment. Acta Biomaterialia 2005, 1, (4), 387-397.

103. Niece, K. L.; Hartgerink, J. D.; Donners, J.; Stupp, S. I., Self-assembly combining two bioactive peptide-amphiphile molecules into nanofibers by electrostatic attraction. Journal of the American Chemical Society 2003, 125, (24), 7146-7147.

104. Bull, S. R.; Guler, M. O.; Bras, R. E.; Meade, T. J.; Stupp, S. I., Selfassembled peptide amphiphile nanofibers conjugated to MRI contrast agents. Nano Letters 2005, 5, (1), 1-4.

105. Pierschbacher, M. D.; Ruoslahti, E., Varients of the cell recognition site of fibronectin that retain attachment-promoting activity. Proceedings of the National Academy of Sciences of the United States of AmericaBiological Sciences 1984, 81, (19), 5985-5988.

106. Ochsenhirt, S. E.; Kokkoli, E.; McCarthy, J. B.; Tirrell, M., Effect of RGD secondary structure and the synergy site PHSRN on cell adhesion, spreading and specific integrin engagement. Biomaterials 2006, 27, (20), 3863-3874.

107. Redick, S. D.; Settles, D. L.; Briscoe, G.; Erickson, H. P., Defining fibronectin's cell adhesion synergy site by site-directed mutagenesis. Journal of Cell Biology 2000, 149, (2), 521-527. 
108. Aota, S.; Nomizu, M.; Yamada, K. M., The short amino-acid-sequence Pro-His-Ser-Arg-Asn in human fibronectin enhances cell-adhesive function. Journal of Biological Chemistry 1994, 269, (40), 24756-24761.

109. Leahy, D. J.; Hendrickson, W. A.; Aukhil, I.; Erickson, H. P., Structure of a fibronectin type-III domain from tenascin phased by MAD analysis of the selenomethionyl protein. Science 1992, 258, (5084), 987-991.

110. Aucoin, L.; Griffith, C. M.; Pleizier, G.; Deslandes, Y.; Sheardown, H., Interactions of corneal epithelial cells and surfaces modified with cell adhesion peptide combinations. Journal of Biomaterials Science-Polymer Edition 2002, 13, (4), 447-462.

111. Huang, F.; Nau, W. M., A conformational flexibility scale for amino acids in peptides. Angewandte Chemie-International Edition 2003, 42, (20), 2269-2272.

112. Benoit, D. S. W.; Anseth, K. S., The effect on osteoblast function of colocalized RGD and PHSRN epitopes on PEG surfaces. Biomaterials 2005, 26, (25), 5209-5220.

113. Kim, T. I.; Jang, J. H.; Lee, Y. M.; Ryu, I. C.; Chung, C. P.; Han, S. B.; Choi, S. M.; Ku, Y., Design and biological activity of synthetic oligopeptides with Pro-His-Ser-Arg-Asn (PHSRN) and Arg-Gly-Asp (RGD) motifs for human osteoblast-like cell (MG-63) adhesion. Biotechnology Letters 2002, 24, (24), 2029-2033.

114. Schmidt, D. R.; Kao, W. J., Monocyte activation in response to polyethylene glycol hydrogels grafted with RGD and PHSRN separated by interpositional spacers of various lengths. Journal of Biomedical Materials Research Part A 2007, 83A, (3), 617-625.

115. Kao, W. J.; Hubbell, J. A.; Anderson, J. M., Protein-mediated macrophage adhesion and activation on biomaterials: a model for modulating cell behavior. Journal of Materials Science-Materials in Medicine 1999, 10, (10-11), 601-605.

116. Susuki, Y.; Hojo, K.; Okazaki, I.; Kamata, H.; Sasaki, M.; Maeda, M.; Nomizu, M.; Yamamoto, Y.; Nakagawa, S.; Mayumi, T.; Kawasaki, K., Preparation and biological activities of a bivalent poly(ethylene glycol) hybrid containing an active site and its synergistic site of fibronectin. Chemical \& Pharmaceutical Bulletin 2002, 50, (9), 1229-1232. 
117. Hiemenz, P. C.; Lodge, T., Polymer chemistry. 2nd ed.; CRC Press: Boca Raton, 2007; p xvii, 587 p.

118. Idiris, A.; Alam, M. T.; Ikai, A., Spring mechanics of alpha-helical polypeptide. Protein Engineering 2000, 13, (11), 763-770.

119. Mardilovich, A.; Craig, J. A.; McCammon, M. Q.; Garg, A.; Kokkoli, E., Design of a novel fibronectin-mimetic peptide-amphiphile for functionalized biomaterials. Langmuir 2006, 22, (7), 3259-3264.

120. Kaiser, E.; Colescott, R.; Bossinger, C.; PI, C., Color test for detection of free terminal amino groups in the solid-phase synthesis of peptides. Analytical Biochemistry 1970, 34, (2), 595-598.

121. Vojkovsky, T., Detection of secondary-amines on solid-phase. Peptide Research 1995, 8, (4), 236-237.

122. Snyder, L. R.; Kirkland, J. J.; Glajch, J. L.; NetLibrary Inc., Practical HPLC method development. In 2nd ed.; Wiley: New York, 1997; pp xxvi, $765 \mathrm{p}$.

123. Franklin, B.; Brownrigg, W.; Farish, M., Of the stilling of waves by means of oil. Extracted from Sundry Letters between Benjamin Franklin, LL. D. F. R. S. William Brownrigg, M. D. F. R. S. and the Reverend Mr. Farish. Philosophical Transactions 1774, 64, 445-460.

124. Rayleigh, The tension of contaminated water-surfaces. Philosophical Magazine 1899, 48, (293), 331-337.

125. Roberts, G. G., Langmuir-Blodgett films. Plenum Press: New York, 1990; p xiv, $425 \mathrm{p}$.

126. Agarwal, V. K.; Mitsuhashi, H.; Takasaki, H., New dipping and rainsing device for Langmuir film deposition. Journal of Physics E-Scientific Instruments 1977, 10, (3), 237-240.

127. Langmuir, I., Constitution and fundamental properties of solids and liquids. II. Liquids. Journal of the American Chemical Society 1917, 39, 1848-1906.

128. Blodgett, K. B., Films built by depositing successive unimolecular layers on a solid surface. Journal of the American Chemical Society 1935, 57, 1007-1022. 
129. Gaines, G. L., Insoluble monolayers at liquid-gas interfaces. Interscience Publishers: New York,, 1966; p xiv, 386 p.

130. Adamson, A. W.; Gast, A. P., Physical chemistry of surfaces. 6th ed.; Wiley: New York, 1997; p xxi, 784 p.

131. Mohwald, H., Phospholipid and phospholipid-protein monolayers at the air/water interface. Annual Review of Physical Chemistry 1990, 41, 441476.

132. Knobler, C. M., Recent developments in the study of monolayers at the air-water-interface. Advances in Chemical Physics 1990, 77, 397-449.

133. Haverstick, K. L. Fundamental behavior of a model biomolecular amphiphile system. Thesis (Ph D ), University of Minnesota, 1999., 1999.

134. KSV Inc. Langmuir and Langmuir-Blodgett Films: What and How. http://www.ksvinc.com/LB.htm

135. Binnig, G.; Quate, C. F.; Gerber, C., Atomic force microscope. Physical Review Letters 1986, 56, (9), 930-933.

136. Radmacher, M.; Tillmann, R. W.; Fritz, M.; Gaub, H. E., From molecules to cells - imaging soft samples with the atomic force microscope. Science 1992, 257, (5078), 1900-1905.

137. Binnig, G.; Rohrer, H.; Gerber, C.; Weibel, E., Surface studies by scanning tunneling microscopy. Physical Review Letters 1982, 49, (1), 57-61.

138. Drake, B.; Prater, C. B.; Weisenhorn, A. L.; Gould, S. A. C.; Albrecht, T. R.; Quate, C. F.; Cannell, D. S.; Hansma, H. G.; Hansma, P. K., Imaging crystals, polymers, and processes in water with the atomic force microscope. Science 1989, 243, (4898), 1586-1589.

139. Bustamante, C.; Rivetti, C.; Keller, D. J., Scanning force microscopy under aqueous solutions. Current Opinion in Structural Biology 1997, 7, (5), 709-716.

140. Braet, F.; Rotsch, C.; Wisse, E.; Radmacher, M., Comparison of fixed and living liver endothelial cells by atomic force microscopy. Applied Physics a-Materials Science \& Processing 1998, 66, S575-S578. 
141. McNally, H. A.; Ben Borgens, R., Three-dimensional imaging of living and dying neurons with atomic force microscopy. Journal of Neurocytology 2004, 33, (2), 251-258.

142. Alessandrini, A.; Facci, P., AFM: a versatile tool in biophysics. Measurement Science \& Technology 2005, 16, (6), R65-R92.

143. Uchihashi, T.; Choi, N.; Tanigawa, M.; Ashino, M.; Sugawara, Y.; Nishijima, H.; Akita, S.; Nakayama, Y.; Tokumoto, H.; Yokoyama, K.; Morita, S.; Ishikawa, M., Carbon-nanotube tip for highly-reproducible imaging of deoxyribonucleic acid helical turns by noncontact atomic force microscopy. Japanese Journal of Applied Physics Part 2-Letters 2000, 39, (8B), L887-L889.

144. Hoh, J. H.; Lal, R.; John, S. A.; Revel, J. P.; Arnsdorf, M. F., Atomic force microscopy and dissection of gap-junctions. Science 1991, 253, (5026), 1405-1408.

145. Arakawa, H.; Umemura, K.; Ikai, A., Protein images obtained by STM, AFM, and TEM. Nature 1992, 358, (6382), 171-\&.

146. Dufrene, Y. F.; Lee, G. U., Advances in the characterization of supported lipid films with the atomic force microscope. Biochimica Et Biophysica Acta-Biomembranes 2000, 1509, (1-2), 14-41.

147. Balashev, K.; Jensen, T. R.; Kjaer, K.; Bjornholm, T., Novel methods for studying lipids and lipases and their mutual interaction at interfaces. Part I. Atomic force microscopy. Biochimie 2001, 83, (5), 387-397.

148. Meyer, G.; Amer, N. M., Novel optical approach to atomic force microscopy. Applied Physics Letters 1988, 53, (12), 1045-1047.

149. Burnham, N. A.; Behrend, O. P.; Oulevey, F.; Gremaud, G.; Gallo, P. J.; Gourdon, D.; Dupas, E.; Kulik, A. J.; Pollock, H. M.; Briggs, G. A. D., How does a tip tap? Nanotechnology 1997, 8, (2), 67-75.

150. Tamayo, J.; Garcia, R., Deformation, contact time, and phase contrast in tapping mode scanning force microscopy. Langmuir 1996, 12, (18), 4430-4435.

151. Tamayo, J.; Garcia, R., Effects of elastic and inelastic interactions on phase contrast images in tapping-mode scanning force microscopy. Applied Physics Letters 1997, 71, (16), 2394-2396. 
152. Hertz, H., Ueber die Berührung fester elastischer Körper. J. Reine Angew. Math. 1882, 92, 156-171.

153. Tao, N. J.; Lindsay, S. M.; Lees, S., Measuring the microelastic properties of biological-material. Biophysical Journal 1992, 63, (4), 11651169.

154. Dufrene, Y. F., Recent progress in the application of atomic force microscopy imaging and force spectroscopy to microbiology. Current Opinion in Microbiology 2003, 6, (3), 317-323.

155. Grandbois, M.; Beyer, M.; Rief, M.; Clausen-Schaumann, H.; Gaub, H. E., How strong is a covalent bond? Science 1999, 283, (5408), 17271730.

156. Merkel, R.; Nassoy, P.; Leung, A.; Ritchie, K.; Evans, E., Energy landscapes of receptor-ligand bonds explored with dynamic force spectroscopy. Nature 1999, 397, (6714), 50-53.

157. Craig, J. A.; Rexeisen, E. L.; Mardilovich, A.; Shroff, K.; Kokkoli, E., Effect of linker and spacer on the design of a fibronectin-mimetic peptide evaluated via cell studies and AFM adhesion forces. Langmuir 2008, 24, (18), 10282-10292.

158. Spoerke, E. D.; Anthony, S. G.; Stupp, S. I., Enzyme directed templating of artificial bone mineral. Advanced Materials 2009, 21, (4), 425-+.

159. Rajangam, K.; Behanna, H. A.; Hui, M. J.; Han, X. Q.; Hulvat, J. F.; Lomasney, J. W.; Stupp, S. I., Heparin binding nanostructures to promote growth of blood vessels. Nano Letters 2006, 6, (9), 2086-2090.

160. Rajangam, K.; Arnold, M. S.; Rocco, M. A.; Stupp, S. I., Peptide amphiphile nanostructure-heparin interactions and their relationship to bioactivity. Biomaterials 2008, 29, (23), 3298-3305.

161. Ghanaati, S.; Webber, M. J.; Unger, R. E.; Orth, C.; Hulvat, J. F.; Kiehna, S. E.; Barbeck, M.; Rasic, A.; Stupp, S. I.; Kirkpatrick, C. J., Dynamic in vivo biocompatibility of angiogenic peptide amphiphile nanofibers. Biomaterials 2009, 30, (31), 6202-6212.

162. Silva, G. A.; Czeisler, C.; Niece, K. L.; Beniash, E.; Harrington, D. A.; Kessler, J. A.; Stupp, S. I., Selective differentiation of neural progenitor cells by high-epitope density nanofibers. Science 2004, 303, (5662), 1352-1355. 
163. Jun, H. W.; Yuwono, V.; Paramonov, S. E.; Hartgerink, J. D., Enzymemediated degradation of peptide-amphiphile nanofiber networks. Advanced Materials 2005, 17, (21), 2612-+.

164. Galler, K. M.; Cavender, A.; Yuwono, V.; Dong, H.; Shi, S. T.; Schmalz, G.; Hartgerink, J. D.; D'Souza, R. N., Self-assembling peptide amphiphile nanofibers as a scaffold for dental stem cells. Tissue Engineering Part $A$ 2008, 14, (12), 2051-2058.

165. Tysseling-Mattiace, V. M.; Sahni, V.; Niece, K. L.; Birch, D.; Czeisler, C.; Fehlings, M. G.; Stupp, S. I.; Kessler, J. A., Self-assembling nanofibers inhibit glial scar formation and promote axon elongation after spinal cord injury. Journal of Neuroscience 2008, 28, (14), 3814-3823.

166. Harrington, D. A.; Cheng, E. Y.; Guler, M. O.; Lee, L. K.; Donovan, J. L.; Claussen, R. C.; Stupp, S. I., Branched peptide-amphiphiles as selfassembling coatings for tissue engineering scaffolds. Journal of Biomedical Materials Research Part A 2006, 78A, (1), 157-167.

167. Jiang, H. Z.; Stupp, S. I., Dip-pen patterning and surface assembly of peptide amphiphiles. Langmuir 2005, 21, (12), 5242-5246.

168. Hung, A. M.; Stupp, S. I., Understanding factors affecting alignment of self-assembling nanofibers patterned by sonication-assisted solution embossing. Langmuir 2009, 25, (12), 7084-7089.

169. Stendahl, J. C.; Rao, M. S.; Guler, M. O.; Stupp, S. I., Intermolecular forces in the self-assembly of peptide amphiphile nanofibers. Advanced Functional Materials 2006, 16, (4), 499-508.

170. Behanna, H. A.; Donners, J.; Gordon, A. C.; Stupp, S. I., Coassembly of amphiphiles with opposite peptide polarities into nanofibers. Journal of the American Chemical Society 2005, 127, (4), 1193-1200.

171. Muraoka, T.; Koh, C. Y.; Cui, H. G.; Stupp, S. I., Light-triggered bioactivity in three dimensions. Angewandte Chemie-International Edition 2009, 48, (32), 5946-5949.

172. Lee, H. K.; Soukasene, S.; Jiang, H. Z.; Zhang, S. M.; Feng, W. C.; Stupp, S. I., Light-induced self-assembly of nanofibers inside liposomes. Soft Matter 2008, 4, (5), 962-964. 
173. Tsonchev, S.; Schatz, G. C.; Ratner, M. A., Hydrophobically-driven selfassembly: A geometric packing analysis. Nano Letters 2003, 3, (5), 623626.

174. Tsonchev, S.; Schatz, G. C.; Ratner, M. A., Electrostatically-directed selfassembly of cylindrical peptide amphiphile nanostructures. Journal of Physical Chemistry B 2004, 108, (26), 8817-8822.

175. Muraoka, T.; Cui, H.; Stupp, S. I., Quadruple helix formation of a photoresponsive peptide amphiphile and its light-triggered dissociation into single fibers. Journal of the American Chemical Society 2008, 130, (10), 2946-2947.

176. Tsai, W. W.; Li, L. S.; Cui, H. G.; Jiang, H. Z.; Stupp, S. I., Self-assembly of amphiphiles with terthiophene and tripeptide segments into helical nanostructures. Tetrahedron 2008, 64, (36), 8504-8514.

177. Tovar, J. D.; Claussen, R. C.; Stupp, S. I., Probing the interior of peptide amphiphile supramolecular aggregates. Journal of the American Chemical Society 2005, 127, (20), 7337-7345.

178. Paramonov, S. E.; Jun, H. W.; Hartgerink, J. D., Modulation of peptideamphiphile nanofibers via phospholipid inclusions. Biomacromolecules 2006, 7, (1), 24-26.

179. Guler, M. O.; Claussen, R. C.; Stupp, S. I., Encapsulation of pyrene within self-assembled peptide amphiphile nanofibers. Journal of Materials Chemistry 2005, 15, (42), 4507-4512.

180. Guler, M. O.; Hsu, L.; Soukasene, S.; Harrington, D. A.; Hulvat, J. F.; Stupp, S. I., Presentation of RGDS epitopes on self-assembled nanofibers of branched peptide amphiphiles. Biomacromolecules 2006, 7, (6), 1855-1863.

181. Paramonov, S. E.; Jun, H. W.; Hartgerink, J. D., Self-assembly of peptide-amphiphile nanofibers: The roles of hydrogen bonding and amphiphilic packing. Journal of the American Chemical Society 2006, 128, (22), 7291-7298.

182. Niece, K. L.; Czeisler, C.; Sahni, V.; Tysseling-Mattiace, V.; Pashuck, E. T.; Kessler, J. A.; Stupp, S. I., Modification of gelation kinetics in bioactive peptide amphiphiles. Biomaterials 2008, 29, (34), 4501-4509. 
183. Jiang, H. Z.; Guler, M. O.; Stupp, S. I., The internal structure of selfassembled peptide amphiphiles nanofibers. Soft Matter 2007, 3, (4), 454462.

184. Sunde, M.; Serpell, L. C.; Bartlam, M.; Fraser, P. E.; Pepys, M. B.; Blake, C. C. F., Common core structure of amyloid fibrils by synchrotron X-ray diffraction. Journal of Molecular Biology 1997, 273, (3), 729-739.

185. Marini, D. M.; Hwang, W.; Lauffenburger, D. A.; Zhang, S. G.; Kamm, R. D., Left-handed helical ribbon intermediates in the self-assembly of a beta-sheet peptide. Nano Letters 2002, 2, (4), 295-299.

186. Tsonchev, S.; Troisi, A.; Schatz, G. C.; Ratner, M. A., All-atom numerical studies of self-assembly of zwitterionic peptide amphiphiles. Journal of Physical Chemistry B 2004, 108, (39), 15278-15284.

187. Levitt, M., Conformational preferences of amino-acids in globular proteins. Biochemistry 1978, 17, (20), 4277-4284.

188. Koehl, P.; Levitt, M., Structure-based conformational preferences of amino acids. Proceedings of the National Academy of Sciences of the United States of America 1999, 96, (22), 12524-12529.

189. Minor, D. L.; Kim, P. S., Measurement of the beta-sheet-forming propensities of amino-acids. Nature 1994, 367, (6464), 660-663.

190. Smith, C. K.; Withka, J. M.; Regan, L., A thermodynamic scale for the beta-sheet forming tendencies of the amino-acids. Biochemistry 1994, 33, (18), 5510-5517.

191. Otzen, D. E.; Fersht, A. R., Side-chain determininants of beta-sheet stability. Biochemistry 1995, 34, (17), 5718-5724.

192. Malkov, S. N.; Zivkovic, M. V.; Beljanski, M. V.; Stojanovic, S. D.; Zaric, S. D., A reexamination of correlations of amino acids with particular Secondary Structures. Protein Journal 2009, 28, (2), 74-86.

193. Voet, D.; Voet, J. G.; Pratt, C. W., Fundamentals of biochemistry upgrade. [Rev. ed.]; Wiley: New York, 2002.

194. Evans, D. F.; Wennerström, H., The Colloidal Domain. VCH Publishers: New York, 1994. 
195. Lau, C.; Bitton, R.; Bianco-Peled, H.; Schultz, D. G.; Cookson, D. J.; Grosser, S. T.; Schneider, J. W., Morphological characterization of selfassembled peptide nucleic acid amphiphiles. Journal of Physical Chemistry B 2006, 110, (18), 9027-9033.

196. Hiemenz, P. C.; Rajagopalan, R., Principles of colloid and surface chemistry. 3rd ed.; Marcel Dekker: New York, 1997; p xix, 650 p.

197. Research, M. Automated Protein Characterization With The MPT-2 Autotitrator. http://www.bioresearchonline.com/article.mvc/AutomatedProtein-Characterization-With-The-M-0002 (2/2/10),

198. Kelly, S. M.; Jess, T. J.; Price, N. C., How to study proteins by circular dichroism. Biochimica Et Biophysica Acta-Proteins and Proteomics 2005, 1751, (2), 119-139.

199. Wikipedia - Circular Polarization.

http://en.wikipedia.org/wiki/File:Polarisation (Circular).svg (2/2/2010)

200. Greenfield, N. J., Methods to estimate the conformation of proteins and polypeptides from circular dichroism data. Analytical Biochemistry 1996, 235, (1), 1-10.

201. Manavalan, P.; Johnson, W. C., Sensitivity of circular dichroism to protein tertiary structure class. Nature 1983, 305, (5937), 831-832.

202. Su, J. Y.; Hodges, R. S.; Kay, C. M., Effect of chain-length on the formation and stability of synthetic alpha-helical coiled coils. Biochemistry 1994, 33, (51), 15501-15510.

203. Manning, M. C.; Woody, R. W., Theoretical determination of the CD of proteins containing closely packed antiparallel beta-sheets. Biopolymers 1987, 26, (10), 1731-1752.

204. Brahms, S.; Brahms, J., Determination of protein secondary structure in solution by vacuum ultraviolet circular-dichroism. Journal of Molecular Biology 1980, 138, (2), 149-178.

205. Bella, J.; Hindle, K. L.; McEwan, P. A.; Lovell, S. C., The leucine-rich repeat structure. Cellular and Molecular Life Sciences 2008, 65, (15), 2307-2333. 
206. Svergun, D. I.; Feigin, L. A.; Taylor, G. W., Structure analysis by smallangle $x$-ray and neutron scattering. Plenum Press: New York, 1987; $p$ xiii, $335 \mathrm{p}$.

207. Sears, V. F., Neutron scattering lengths and cross sections. Neutron News 1992, 3, (3), 29-37.

208. Guinier, A.; Fournet, G., In Small Angle Scattering of X-Rays, Mayer, M. G., Ed. J. Wiley \&amp; Sons: New York, 1955.

209. Glinka, C. J.; Barker, J. G.; Hammouda, B.; Krueger, S.; Moyer, J. J.; Orts, W. J., The $30 \mathrm{~m}$ small-angle neutron scattering instruments at the National Institute of Standards and Technology. Journal of Applied Crystallography 1998, 31, 430-445.

210. Hammouda, B., A tutorial on small-angle neutron scattering from polymers. In National Institute of Standards and Technology: Gaithersburg, MD, 1995; http://www.ncnr.nist.gov/programs/sans/pdf/polymer tut.pdf

211. Ferry, J. D., Viscoelastic properties of polymers. 3d ed.; Wiley: New York, 1980; p xxiv, 641 p. 